\title{
Structural and Material Exploration of Magnetic Tunnel Junctions
}

\author{
Wei Chen \\ Changzhou, Jiangsu, China
}

Bachelor of Science, Nanjing University, 2004

A Dissertation presented to the Graduate Faculty of the University of Virginia in Candidacy for the Degree of Doctor of Philosophy

Department of Physics

University of Virginia

May, 2010

Approved by the

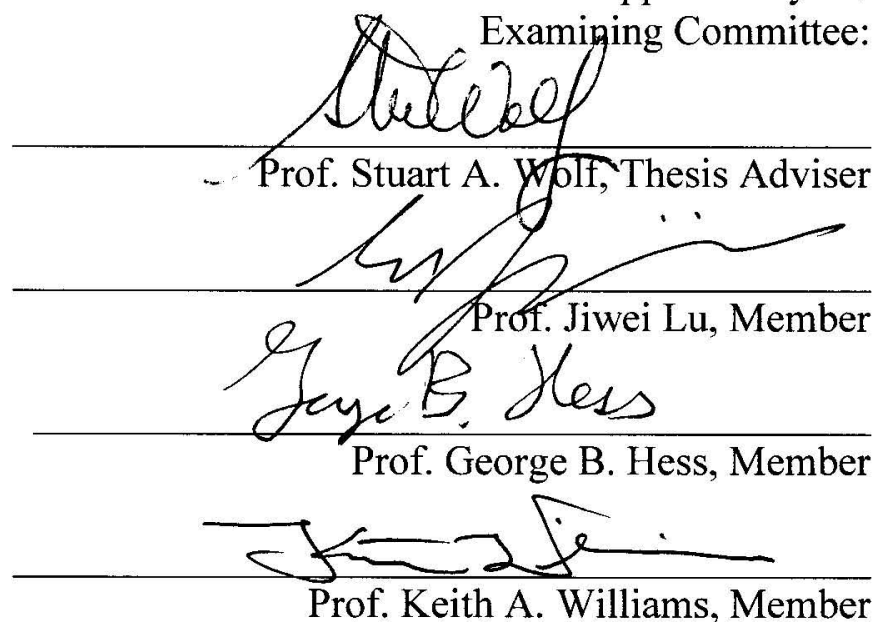




\section{CONTENTS}

Structural and Material Exploration of Magnetic Tunnel Junctions ............................... i

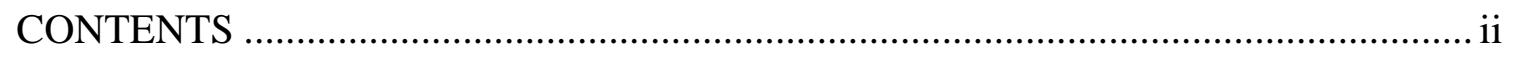

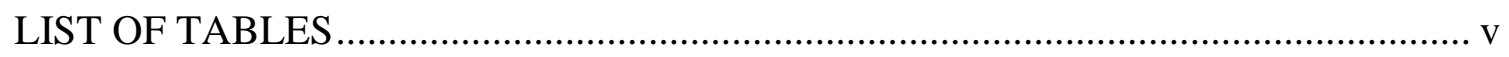

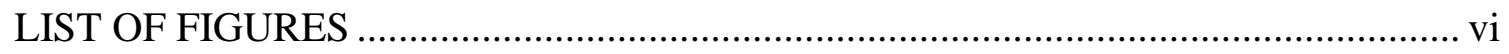

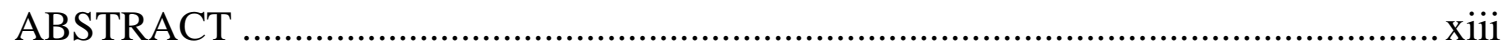

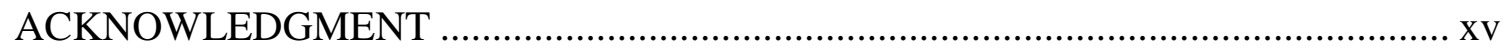

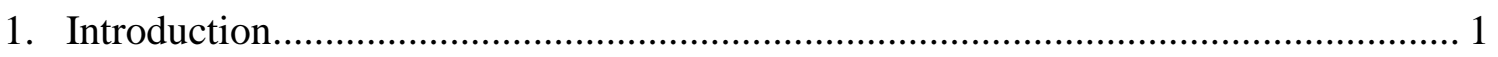

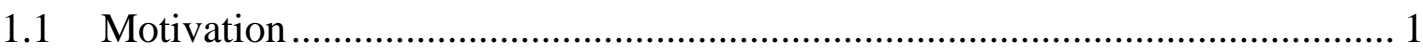

1.2 An overview of the dissertation .............................................................. 4

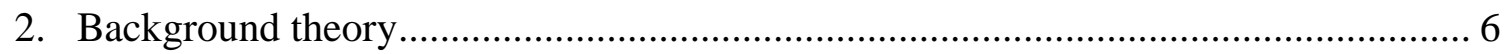

2.1 Spin polarized transport in magnetic nanostructures ................................. 6

2.1.1 Itinerant ferromagnetism ......................................................... 6

2.1.2 Spin polarized transport in magnetic nanostructures ........................ 9

2.2 Giant and Tunneling magnetoresistance ................................................. 10

2.2.1 Giant magnetoresistance (GMR) ............................................. 10

2.2.2 Tunneling magnetoresistance (TMR) ......................................... 13

2.3 Magnetic tunnel junctions (MTJs) ......................................................... 14

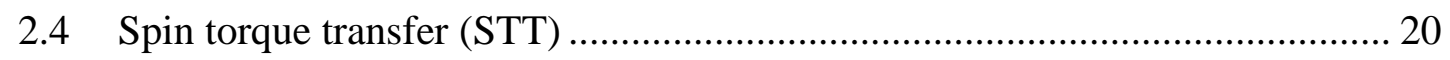

3. Experimental techniques and sample fabrication ............................................. 25

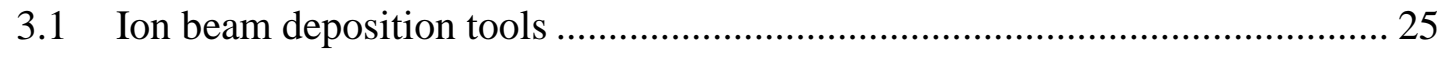

3.1.1 Biased target ion beam deposition (BTIBD) ................................. 25

3.1.2 Reactive biased target ion beam deposition (RBTIBD).................... 31

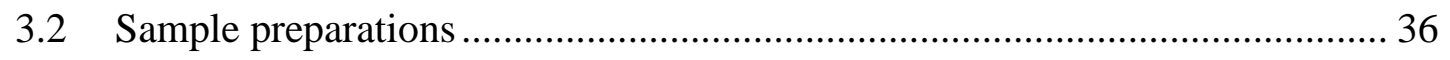

4. Exchange bias for magnetic tunnel junctions ............................................... 40

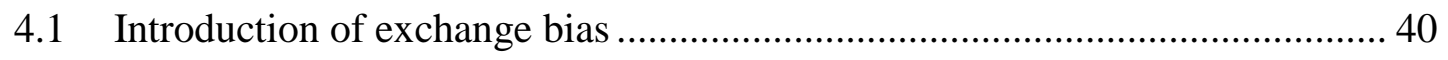


4.2 Exchange bias in $\mathrm{CoFe} / \mathrm{FeMn} / \mathrm{CoFe}$ trilayers .............................................. 43

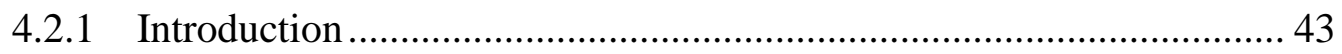

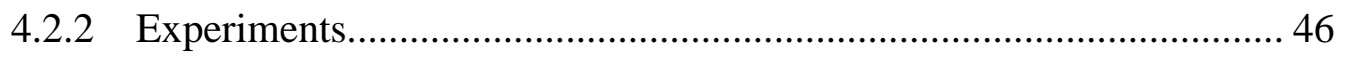

4.2.3 Results and discussions .................................................................. 46

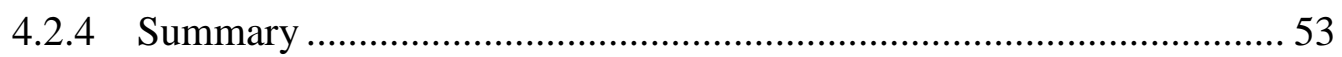

5. Oxidation of the ferromagnetic bottom electrode in magnetic tunnel junctions ........ 54

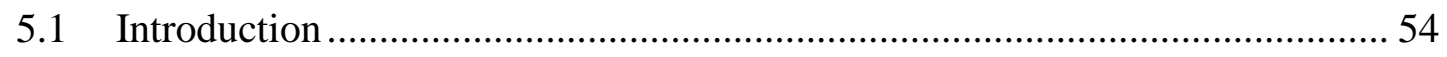

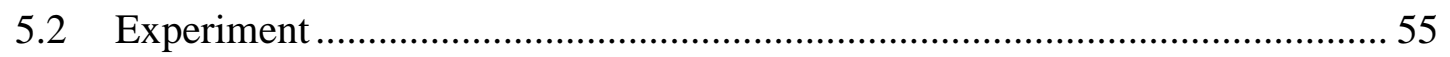

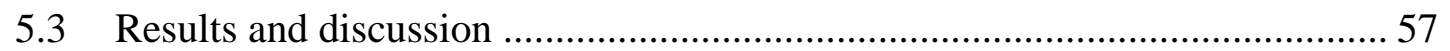

5.3.1 Parameters related to EB effect......................................................... 57

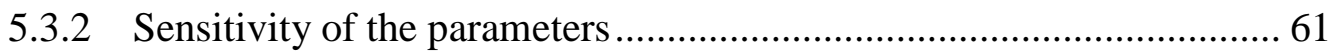

5.3.3 Annealing effect on detection parameters ........................................... 63

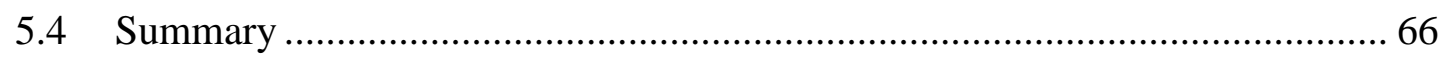

6. Interface engineering: effects of target bias voltage on magnetic tunnel junctions

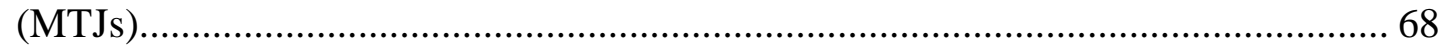

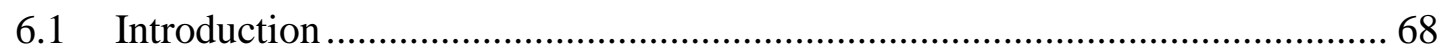

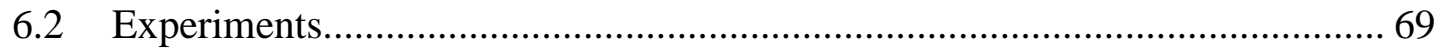

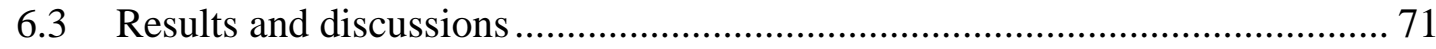

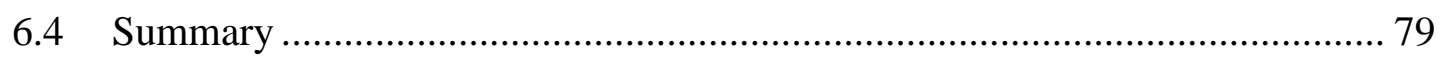

7. Magnetic tunnel junctions with $\mathrm{MgO}$ tunnel barrier by bias target ion beam

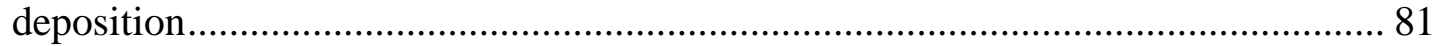

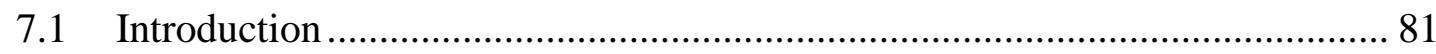

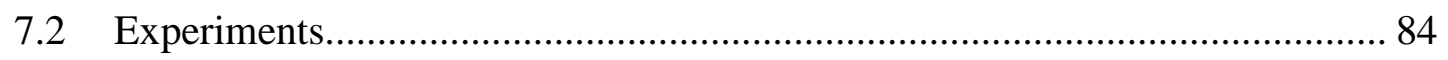

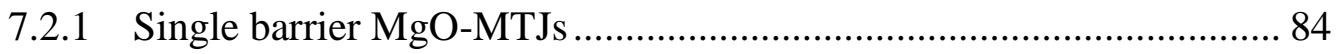

7.2.2 Double barrier MgO-MTJs ………………………………………….... 87

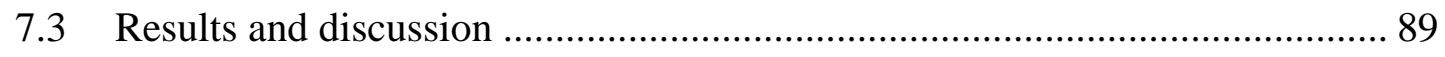

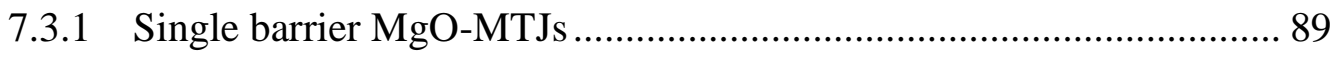

7.3.2 Double barrier MgO-MTJs ................................................................ 97 


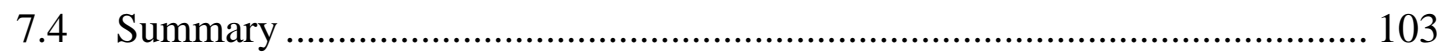

8. Exploration of $\tau-\mathrm{MnAl}$ as electrodes for MTJs ................................................. 105

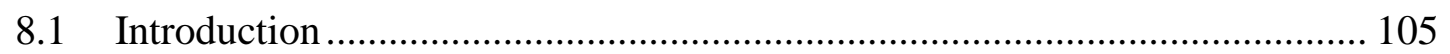

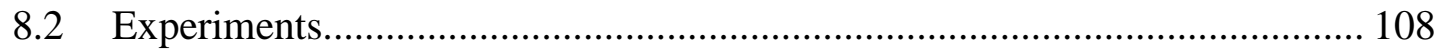

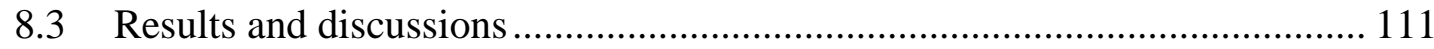

8.3.1 Selection of substrate templates ............................................. 111

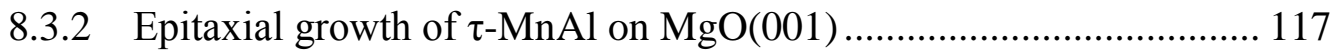

8.3.3 Composition dependence of $\tau$-MnAl properties ........................... 120

8.3.4 Correlation between chemical ordering and saturation magnetization 122

8.3.5 Annealing effects on $\tau$-MnAl properties .................................. 128

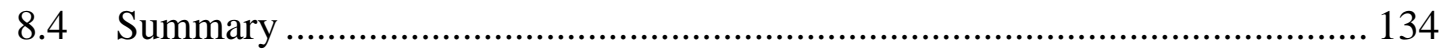

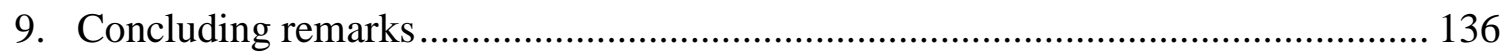

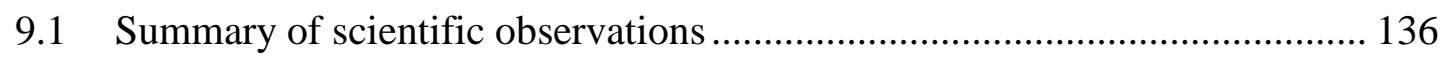

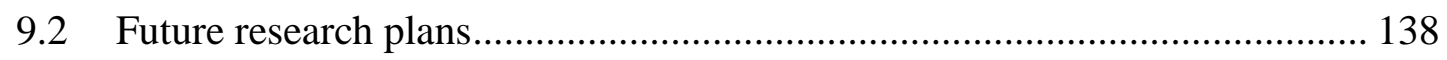

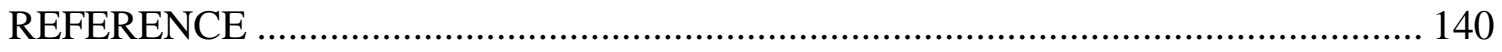




\section{LIST OF TABLES}

Table 7-1 Effect of $\mathrm{MgO}$ barrier oxidation conditions on TMR value. Sample set was

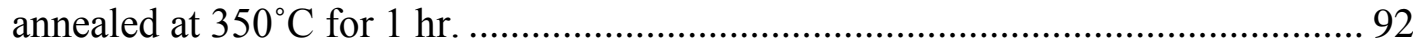

Table 8-1 Typical process conditions for MnAl and Ta film deposition by RBTIBD .. 110

Table 8-2 Saturation magnetization of MnAl (1hr deposition) on different substrates. Here $\mathrm{MgO}(\mathrm{BTIBD})$ refers to $6 \AA$ of $\mathrm{Mg}$ deposited by BTIBD on a thermally oxidized $\mathrm{Si}$ substrate, followed by natual oxidation when exposed in air before being put in the RBTIBD as a growth template; $\mathrm{SiO}_{2}$ refers to a thermally oxidized Si substrate with a $\sim 3000 \AA \mathrm{SiO}_{2}$ oxidation layer. All other substrates are commercially available products from various suppliers.

113

Table 8-3 Annealing parameters of two vacuum furnace (A) regular (B) rapid thermal annealing

Table 8-4 Comparison of the two annealing methods (regular annealing and rapid thermal annealing) on two representative samples with different thickness 


\section{LIST OF FIGURES}

Figure 1.1 The maximum current that can be provided by a CMOS transistor as a function of its size is plotted as the gray line. The current necessary to switch MRAM using a magnetic field produced by crossed wires is plotted as the black line. The lines defined by the various symbols are the switching currents as a function of MTJ size for various values of the current density. Note that for a reasonable margin a current density of $5 \times 10^{5} \mathrm{~A} / \mathrm{cm}^{2}$ for $45 \mathrm{~nm}$ junctions can be provided by a CMOS transistor of the same size! We are swiftly approaching this size as indicated by the scale on the top showing the expected year that CMOS will reach the various scales. (Adapted from NSF EAGAR Proposal, Prof. Stuart A.

Wolf, UVA 2009) 3

Figure 2.1 (LEFT) a schematic plot is shown for the energy band structure of a d transition metal. The density of states $\mathrm{N}(\mathrm{E})$ is shown separately for the spin up and down electrons and where a simplified separation has been made between the $4 \mathrm{~s}$ and $3 \mathrm{~d}$ band energies. For the non-magnetic state these are identical for the two spins. All energy levels below the Fermi energy are occupied states (orange and blue). The colored area (orange + blue) corresponds to the total number of conduction electrons in the metal. (RIGHT) the corresponding picture is illustrated for a ferromagnetic state, with a spin-polarization chosen to be in the up direction $(\mathrm{N} \uparrow>\mathrm{N} \downarrow$; blue area $>$ orange area). This polarization is indicated by the thick blue arrow at the bottom figure to the right. (Reproduced from Ref. [7])....................... 8

Figure 2.2 Spin polarized electron transport through the Nonmagnetic/Ferromagnetic/Nonmagnetic metal structure. (Adapted from the NRI e-workshop: "Spin Torque Transfer" 08/2009).

Figure 2.3 Two-current mode of the giant magnetoresistance effect in the F/N/F spin valve structure. (a) In the parallel (P) magnetization configuration, the equivalent resistor circuit (b) gives $R_{P}=2 R_{\uparrow} R_{\downarrow} /\left(R_{\uparrow}+R_{\downarrow}\right)$, while in the antiparallel (AP) configuration (c), the resistor circuit (d) gives $\mathrm{R}_{\mathrm{AP}}=\left(\mathrm{R}_{\uparrow}+\mathrm{R}_{\downarrow}\right) / 2>\mathrm{R}_{\mathrm{P}}$. (Adapted from Ref. [12]) 
Figure 2.4 Schematics of electron tunneling in ferromagnet/insulator/ferromagnet (F/I/F) tunnel junctions: (a) Parallel and (b) antiparallel orientation of magnetizations with the corresponding spin-resolved density of the $\mathrm{d}$ states in ferromagnetic metals that have exchange spin splitting $\Delta_{\mathrm{ex}}$. Arrows in the two ferromagnetic regions are determined by the majority-spin sub-band. Dashed lines depict spinconserved tunneling. (Reproduced from Ref. [9]).

Figure 2.5 The evolution of MTJ structures: (a) MJTs with single ferromagnetic free layer (FL) whose magnetization could be rotated to achieve high and low resistance state, and the reference layer (RL) whose magnetization is fixed. (b) MTJs with exchange biased RL. (c) Exchange biased artificial antiferromagnet (AAF) as the RL. (d) Exchange biased AAF as the RL and artificial ferromagnet (AFi) as the FL of MTJs. (Adapted from Ref. [25]) 16

Figure 2.6 Read and program mode of a MRAM unit cell with 1Transistor-1MTJ architecture. In the read mode, the transistor is turned on, and a sensing current is passing through the bit line to the read out the resistance state of the MTJ; In the program mode, the transistor is turned off, a program current passed through the same bit line as well as the digit line to generate magnetic field to switch the orientation of the free layer of MTJ in order to change its resistance state. (Adapted from Spintronics lecture notes, Prof. Stu Wolf, UVA 2006) 18

Figure 2.7 Spin Torque Transfer (STT) inside a spin valve or magnetic tunnel junction scheme. When an electron current passes through the ferromagnetic fixed layer, it becomes spin polarized and when flowing through the second ferromagnetic free layer, it exerts a spin torque on the magnetic moment of the free layer due to the interaction between the electron spin and local magnetization. (Adapted from Spintronics lecture notes, Prof. Stu Wolf, UVA 2006) ...................................... 20

Figure 3.1 Schematic illustration of the process chamber of the BTIBD system (top view, adapted from Ref. [39]). 27

Figure 3.2 Schematic layout of Mark II End-Hall ion source and HCES 5000 Hollow Cathode electron source. (Adapted from Ref. [39]) 30

Figure 3.3 The schematic illustration of the RBTIBD processing chamber. (Adapted from Ref. [33]) 
Figure 3.4 The schematic illustration of the pulsed dc bias control. (Adapted from Ref. [33]) 33

Figure 4.1 (a) Representative MTJ stack with exchange biased fixed layer; (b) $\mathrm{M}(\mathrm{H})$ hysteresis loop of the MTJ stack showing the separate switching of the free layer and exchange biased fixed layer. 41

Figure 4.2 Schematic diagram of the spin configuration of an FM/AFM bilayer at different stage (i)-(v) of an exchange biased hysteresis loop. Note that the spin configurations are just a simple cartoon to illustrate the effect of the coupling and they are not necessarily accurate portraits of the actual rotation of the FM or AFM magnetizations. (Adapted from Ref. [26]).

Figure 4.3 M(H) loops of the bilayer structure $\mathrm{Si} / \mathrm{Cu}(5) / \mathrm{FeMn}(10) / \mathrm{CoFe}(4) / \mathrm{Ta}(5)$ (a) and (d); $\quad \mathrm{Si} / \mathrm{Ta}(5) / \mathrm{CoFe}(4) / \mathrm{FeMn}(10) / \mathrm{Cu}(5) \quad$ (b) and $\mathrm{Si} / \mathrm{Ta}(5) / \mathrm{CoFe}(4) / \mathrm{FeMn}(10) / \mathrm{CoFe}(4) / \mathrm{Ta}(5)$ trilayers (c) and (f). Samples (a) (b) and (c) were as-deposited, and samples (d) (e) (f) were field cooled. The crosses marked the center of the main or minor loops where $\mathrm{H}_{\mathrm{EB}}$ was determined......................... 48 Figure 4.4 M(H) loops of the bilayer structure $\mathrm{Si} / \mathrm{Cu}(5) / \mathrm{FeMn}(6) / \mathrm{CoFe}(4) / \mathrm{Ta}(5)$ (a) and (d); $\quad \mathrm{Si} / \mathrm{Ta}(5) / \mathrm{CoFe}(4) / \mathrm{FeMn}(6) / \mathrm{Cu}(5) \quad$ (b) and $\mathrm{Si} / \mathrm{Ta}(5) / \mathrm{CoFe}(4) / \mathrm{FeMn}(6) / \mathrm{CoFe}(4) / \mathrm{Ta}(5)$ trilayers (c) and (f). Samples (a) (b) and (c) were as-deposited, and samples (d) (e) (f) were field cooled. The crosses marked the center of the main or minor loops where $\mathrm{H}_{\mathrm{EB}}$ was determined......................... 50

Figure 5.1 RT and $11 \mathrm{~K}$ hysteresis loop measurement of the sample with (a) CoFe (b) $\mathrm{CoFeB}$ bottom electrode and both with 90 seconds $\mathrm{MgO}$ barrier oxidation. Also shown is the corresponding reference sample (in black) with the same structure as the oxidized samples in (a) and (b) except no plasma oxidation of the Mg metal layer. The inset of (b) shows the general structure of the partial MTJ stack use in

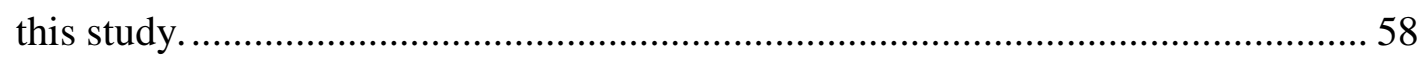

Figure 5.2 The oxidation time dependence of the four detection parameters $\mathrm{H}_{\mathrm{eb}}, \mathrm{H}_{\mathrm{c}}$, TE ratio and UC spin density. Points in black are from the samples with CoFe bottom electrodes and red points represent the data from samples with $\mathrm{CoFeB}$ electrodes. All measurements were done at $11 \mathrm{~K}$ 62 
Figure 5.3 The annealing effect on the four detection parameters for sample sets with $\mathrm{CoFeB}$ bottom electrode. Data points in black are from the as-deposited samples, and blue and red points represent the data from samples annealing at $200^{\circ} \mathrm{C}$ and $350^{\circ} \mathrm{C}$, respectively. All measurement was done at $11 \mathrm{~K}$ 64

Figure 5.4 The normalized data of $\mathrm{H}_{\mathrm{c}}$ including the results after annealing for $1 \mathrm{hr}$ at two different temperatures $\left(200^{\circ} \mathrm{C}\right.$ and $\left.350^{\circ} \mathrm{C}\right)$, as well as the as-deposited data. The normalization was done by dividing the absolute values of $\mathrm{H}_{\mathrm{c}}$ by the corresponding values at RT. 66

Figure 6.1 Schematic illustration of the typical MTJ stack 70

Figure 6.2 Representative TMR result from the CIPT measurement. (Adapted from Ref. [70]) 72

Figure 6.3 Representative loop center shifts of both free and pinned layers at different temperatures. (Adapted from Ref. [70]) 73

Figure 6.4 Schematic illustration of Néel coupling model. As the two dominant interlayer coupling mechanisms, $\mathrm{H}_{\mathrm{M}}$ and $\mathrm{H}_{\mathrm{N}}$ represent the magnetostatic coupling and the Néel coupling respectively. Here in our case of un-patterned films, $\mathrm{H}_{M}$ is negligible and only $\mathrm{H}_{\mathrm{N}}$ needs to be considered. (Adapted from Ref. [66]) 74

Figure 6.5 The dependence of free layer loop center shift on the target bias voltage used for the free layer deposition. (Adapted from Ref. [70]) 76

Figure 6.6 The dependence of TMR and RA values on the target bias voltage applied for the free layer deposition. (Adapted from Ref. [70]) 77

Figure 6.7 (a) Atomic configurations of $\mathrm{Cu} / \mathrm{Ni} / \mathrm{Cu}$ multilayers deposited with different incident adatom energies simulated by molecular dynamics. (Adapted from Ref. [41]) (b) AFM images of single CoFe layer deposited by BTIBD with different bias voltages. 78

Figure 7.1 Schematic illustrations of spin polarized electron tunneling through (a) an amorphous $\mathrm{Al}_{2} \mathrm{O}_{3}$ barrier and (b) a crystalline $\mathrm{MgO}(001)$ barrier, reproduced from Ref. [27]. 82

Figure 7.2 Material stack of double barrier MTJ with $\mathrm{MgO}$ barriers (units in $\mathrm{nm}$ ). ...... 88

Figure 7.3 M(H) loop at RT of typical MTJ stack with $\mathrm{MgO}$ barrier. 89 
Figure 7.4 TMR vs magnetic field of a representative MgO-MTJ sample after 350C 1 hour annealing. 90

Figure 7.5 MgO barrier thickness dependence of TMR values. The MTJ sample set was quickly field cooled at $280^{\circ} \mathrm{C}$ for $1 \mathrm{~min}$ to set the bottom $\mathrm{EB}$ pinning. ................. 91

Figure 7.6 Annealing effect of MgO-MTJs with different barrier thickness. ................ 94

Figure 7.7 Annealing effect of MgO-MTJs with different CoFeB layer thickness........ 96

Figure 7.8 Illustration of spin torque transfer (STT) switching mechanism for single barrier MTJ with two different initial states, with the pinned layer and free layer either parallel or antiparallel to each other before the STT switching. 98

Figure 7.9 Illustration of STT switching mechanism for double barrier MTJ (two pinned layers are configured antiparallel to each other) with two different initial states. .. 99 Figure 7.10 Illustration of STT switching mechanism for double barrier MTJ (two pinned layers are configured parallel to each other) with two different initial states. 100

Figure $7.11 \mathrm{M}(\mathrm{H})$ loop of DBMTJ with two parallel pinned layers.......................... 101

Figure $7.12 \mathrm{M}(\mathrm{H})$ loop of DBMTJ with two antiparallel pinned layers...................... 102

Figure 8.1 (a) Schematic of $\tau$-MnAl structure, the magnetic moments of the Mn atoms are parallel to the c-axis of the $\mathrm{MnAl}$, (b) part of the $\mathrm{Mn}-\mathrm{Al}$ phase diagram showing the $\gamma, \gamma_{2}, \tau, \beta$ and $\varepsilon$-phases. 105

Figure 8.2 Proposed phase transformation sequence and the lattice relationship during the formation of $\tau$ phase MnAl according to Ref. [87]. 107

Figure 8.3 MvsH loops for $1 \mathrm{hr}$ deposition of MnAl thin films on different substrates: (a) $\mathrm{SiO} 2$ (thermally oxidized $\mathrm{Si}$ wafer) (b) $\mathrm{MgO}(\mathrm{BTIBD})$ (c) $\mathrm{SrTiO} 3$ (100) (d)

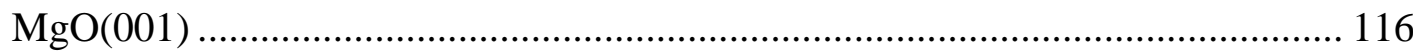

Figure 8.4 XRD 2-Theta/Omega scan of both as-deposited MnAl sample L100409 on $\mathrm{MgO}(001)$ substrate and the same sample after annealing $\left(400^{\circ} \mathrm{C} 30 \mathrm{~min}\right) \ldots \ldots \ldots . . .117$

Figure 8.5 The epitaxial growth of $\mathrm{Fe}(001)$ on $\mathrm{MgO}(001)$ template due to close the lattice matching. It also shows clearly the $45^{\circ}$ offset of the growth orientation

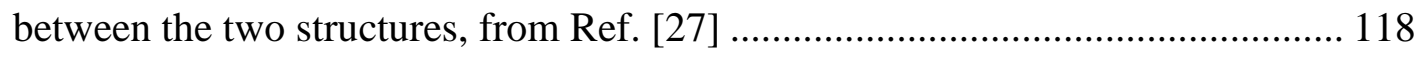

Figure $8.6360^{\circ} \Phi$ scan of $\mathrm{MgO}(220)$ and $\mathrm{MnAl}$ (110) peaks measured by XRD, showing the $45^{\circ}$ offset 119 
Figure 8.7 (a) Taken from Ref. [82], the phase diagram of MnAl alloy in the bulk form. The $\tau$ phase MnAl can only exist with Mn atomic concentration from 50 to $60 \%$. (b) Taken from Ref. [84], the saturation magnetization dependence on the Mn atomic concentration, with highest $\mathrm{M}_{\mathrm{S}} \sim 400 \mathrm{emu} / \mathrm{cc}$ obtained with a $\mathrm{Mn}$ concentration around $50 \%$. 120

Figure 8.8 The saturation magnetization dependence on $\mathrm{Mn}$ atomic concentration from the MnAl samples prepared by RBTIBD. Samples were annealed at $400^{\circ} \mathrm{C}$ for 30 min before the measurement. 122

Figure 8.9 Hysteresis loops of sample L092409 with both in-plane and out-of-plane measurement. 123

Figure 8.10 High resolution XRD scan of MnAl sample L092409 124

Figure 8.11 The chemical ordering $\mathrm{S}$ and saturation magnetization $\mathrm{M}_{\mathrm{S}}$ dependence on the $\tau$-MnAl film thickness 126

Figure 8.12 The illustration of the disordering process of $\tau$-MnAl by swap of the $\mathrm{Mn}$ and $\mathrm{Al}$ atom positions, with the wrong positioned Mn antiferromagnetically coupled with the rest Mn matrix 127

Figure $8.13 \mathrm{M}(\mathrm{H})$ loops of a representative $\mathrm{MnAl}$ thin film sample both before and after $400^{\circ} \mathrm{C} 30$ min annealing treatment 128

Figure 8.14 RTA temperature dependence of two sets of MnAl thin film samples with different thicknesses. The annealing time was kept at $1 \mathrm{~min}$. 132

Figure 8.15 RTA time dependence of two sets of MnAl thin film samples with different thicknesses. The annealing temperature was kept at $400^{\circ} \mathrm{C}$. 133 


\section{List of Abbreviations}

1. BTIBD: Bias Target Ion Beam Deposition

2. EB: Exchange Bias

3. FM: Ferromagnetic / Ferromagnet

4. DBMTJs: Double Barrier Magnetic Tunnel Junctions

5. DOS: Density of States

6. DRAM: Dynamic Random Access Memory

7. GMR: Giant Magneto-Resistance

8. MR: Magneto-Resistance

9. MRAM: Magnetic Random Access Memory

10. MTJs: Magnetic Tunnel Junctions

11. PMA: Perpendicular Magnetic Anisotropy

12. RBTIBD: Reactive Bias Target Ion Beam Deposition

13. RTA: Rapid Thermal Annealing

14. STT: Spin Torque Transfer

15. STT-RAM: Spin Torque Transfer Random Access Memory

16. TMR: Tunneling Magneto-Resistance 


\begin{abstract}
Spin-Torque-Transfer Magnetic Random Access Memory (STT-RAM) has the potential to become a universal memory due to its superior properties compared with conventional memory technologies. However, numerous challenges still remain before this technology becomes practical. Efforts to improve the performance of magnetic tunnel junctions (MTJs), the building block of STT-RAM, were made both from the structural and material aspects, with the results and discussions presented in this dissertation.
\end{abstract}

MTJ structures with $\mathrm{AlO}_{\mathrm{x}}$ tunnel barriers were deposited using the Bias Target Ion Beam Deposition (BTIBD) technique. A CoFe/FeMn/CoFe trilayer with exchange bias (EB) coupling was developed and the underlying physics was studied. By controlling the sputtering voltage, the ferromagnetic (FM) electrode/barrier interface profiles could be tuned to improve the tunneling efficiency as well as other important electrical and magnetic properties. During the MTJ fabrication process, formation of the tunnel barrier is the most critical step. An effective way of detecting the bottom electrode oxidation was developed by simple low temperature magnetic measurement. MTJs using $\mathrm{CoFeB} / \mathrm{MgO} / \mathrm{CoFeB}$ were successfully fabricated by BTIBD with tunneling magnetoresistance (TMR) around 70\%. MTJ performance was optimized by tuning the annealing conditions and other junction stack parameters. Preliminary work on doublebarrier MTJs (DBMTJs) demonstrated the capability of BTIBD to make DBMTJs with the two pinned layers either parallel or antiparallel for different applications.

From a material perspective, MnAl thin film with PMA and other unique properties was explored for its potential application as the FM electrode for MTJs. The successful 
growth of magnetic $\tau$-MnAl with the $\mathrm{L}_{0}$ structure and a record high saturation magnetization value on $\mathrm{MgO}(001)$ substrate confirmed the candidacy of $\mathrm{MnAl}$ as a promising MTJ FM electrode. Further magnetic and structural analysis of $\tau$-MnAl revealed certain correlations between the chemical ordering and saturation magnetization. Post-deposition heat treatment, rapid thermal annealing (RTA) in particular, was found to be a very effective way of tuning the properties of thin-film $\tau$ MnAl. 


\section{ACKNOWLEDGMENT}

The completion of my dissertation and the underlying graduate research work would not be possible without the help and support of many people. First and foremost I would like to thank my advisor Prof. Stuart A. Wolf, for his unconditional support and outstanding mentorship throughout my graduate research career. Next, I would like to thank Prof. Jiwei Lu and Dr. Nam N. H. Dao for their professional hands-on guidance at the initial conduct of my research, and the stimulating discussion and advices throughout my whole graduate research period. I would like to thank all of the former and present members of my research group, including Junjie Quan, Kevin West, Jiani Yu, Yonghang Pei, Wenjing Yin, Melissa Commisso Dolph, David Kirkwood, Wenbin Fan, Yishen Cui, Ryan Comes and Salinporn Kittiwatanakul for making our lab such an enjoyable place to work every day. They are not only good team workers but also good friends in life. I also would like to acknowledge all of the members of the faculty and staff of NanoStar, Physics Department and IPM, particularly Beth Beal, Sherri Sullivan, Suzie Garrett, Tammie Shifflett, Dawn Shifflett, Vicky Ingram, Beth Orser, Tommy Eanes, David Glover, Lisa Friedersdorf, Phil Parrish, Katherine Van Deusen, Teresa Morris, Peter Schare and Rich Gregory for all the supporting work that they have done these years to my research. I would like to express my deep appreciation to my collaborator Dr. Bill Egelhoff in NIST for his help on sample measurement as well as helpful discussions in the past two years, and similar appreciation goes to Dr. Paul Adl at Micron for his help on experiments as well. Also, I would like to thank members of

4Wave Inc, including Sami Antrazi, Trey Middleton, Karl McHenry, David Baldwin and Tony Githinji, for their support on the deposition equipment. Finally and most 
importantly, the unconditional love from my family has been the strongest support for my pursuit of graduate study and the $\mathrm{PhD}$ degree abroad. In particular, I would like to thank my dearest wife Jing for her selfless love and support. The past five years in Charlottesville with her company would be one chapter of my life that I cherish the most. To Jing, our unborn baby, and my dear parents, I dedicate this dissertation work. 


\section{Introduction}

\subsection{Motivation}

In 2006 Freescale Semiconductor introduced the industry's first commercial Magnetic Random Access Memory (MRAM) product. The development of this novel non-volatile magnetic memory from a single prototype device to a fully engineered storage system took approximately 10 years and is regarded as a prime example of innovative engineering in materials, device fabrication, design, architecture and integration. The core component of this new memory technology serving as the storage unit cell is a magnetic tunnel junction (MTJ), which was first demonstrated as a practical device in 1995 [1, 2]. The basic structure of the MTJ is two ferromagnetic layers sandwiching an ultra thin $(1 \sim 2 \mathrm{~nm})$ insulating barrier layer. Initially aluminum oxide was the tunnel barrier material of choice due to its good tunneling properties and simple fabrication. The resistance of the whole sandwich stack depends on the relative orientation of the magnetic moments in the two ferromagnetic layers. When the moments of the two layers are parallel, the stack shows a relative low resistance compared to when the two moments are antiparallel. The figure of merit for this resistance change is the magnetoresistance ratio (MR) that is defined as the ratio of the change in resistance to the relative low resistance when the two moments are parallel, and in the original demonstrated MTJs the MR was only about $10 \%$.

Remarkable progress has been made since the initial demonstration of practical MTJs in 1995. However, there are still many engineering obstacles to overcome before MRAM could successfully scale to the much higher densities that are necessary in order 
to compete with current mainstream non-volatile semiconductor technologies such as FLASH memory.

Conventional MRAM product with toggle switching mode [3] uses external magnetic field to switch the MTJs in the writing process. The field is generated by passing current through external wires and the current required to generate the field becomes too large to be practical when the MTJ cell size becomes smaller. This scalability problem prevented the conventional MRAM products from reaching high enough densities to compete directly with other mature non-volatile semiconductor memories, especially FLASH. Fortunately, a new method of switching called Spin Torque Transfer Switching (STT), which utilizes the torque formed when spin polarized current entering the ferromagnetic materials to switch the moments of the ferromagnet [4, 5], provided a new routine to overcome the restrictions faced by conventional MRAM products. This new memory technology called STT-RAM has the correct scaling behavior, in that it is the current density rather than the absolute current that is important for switching, so the current scales with the device area. However, with current MTJ materials, the critical current density required to switching the MTJ successfully is still too high to be practical, and more innovations are needed in material and structural engineering of MTJs before the high current density could be reduced and the STT switching method could be incorporated into a commercial memory system. As illustrated in Figure 1.1, in order to synchronize with the size of the CMOS transistor that provides the switching current, the current density needs to be lower than $5 \times 10^{5} \mathrm{~A} / \mathrm{cm}^{2}$. The work presented in this dissertation involves both material and structural exploration 
of MTJs with the purpose of improved MTJ performance to achieve lower switching current density and higher packing density of STT-RAM in the near future.

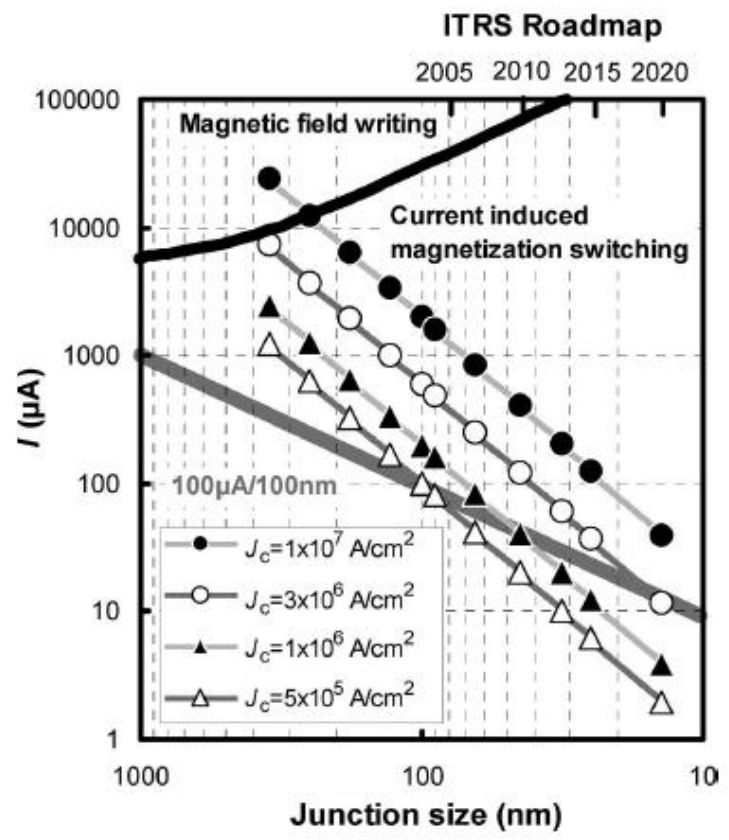

Figure 1.1 The maximum current that can be provided by a CMOS transistor as a function of its size is plotted as the gray line. The current necessary to switch MRAM using a magnetic field produced by crossed wires is plotted as the black line. The lines defined by the various symbols are the switching currents as a function of MTJ size for various values of the current density. Note that for a reasonable margin by a factor of 4 , a current density of $5 \times 10^{5} \mathrm{~A} / \mathrm{cm}^{2}$ for $45 \mathrm{~nm}$ junctions can be provided by a CMOS transistor of the same size! We are swiftly approaching this size as indicated by the scale on the top showing the expected year that CMOS will reach the various scales. (Adapted from NSF EAGAR Proposal, Prof. Stuart A. Wolf, UVA 2009) 


\subsection{An overview of the dissertation}

The central topic of this dissertation is the thin film multilayer structure called magnetic tunnel junctions. Motivated by its great potential in becoming the core component of the next generation universal memory, multiple approaches were applied to improve the electrical and magnetic performance of MTJs for practical applications. Chapter 2 introduces the related theories and principles involved in the MTJs operation, including the background of basic magnetism, the spin polarized tunneling in magnetic materials, and the spin torque transfer (STT) theory, which is introduced here as a new approach to overcome the technical challenges faced by conventional switching mechanism of MTJs. The main purpose of this dissertation is to conduct the proposed research in order to overcome some remaining challenges both material and structural, in order to further push the STT technology for practical applications. Chapter 3 introduces the ion beam deposition technique and the two tools that were used for making the MTJs stacks. It also describes in great detail how I prepared the samples. Chapter 4 through 7 describes systematically how high quality MTJs were made, and how the MTJ performance was improved by optimizing the processing conditions as well as designing new MTJ structures. Chapter 4 focuses on one of the key components of the MTJ structure, which is the magnetically hard layer pinned by the exchange bias coupling as the reference layer. In chapter 5, a simple but effective way is proposed to help quickly identify the process window during the tunnel barrier oxidation formation. This is because the most critical and most challenging processing step during the fabrication of a MTJ stack is the formation of the ultra thin tunnel barrier (usually less than $2 \mathrm{~nm}$ ). In Chapter 6, to further improve the MTJ performance, a method was proposed to optimize 
the quality of interfaces between the tunnel barrier and the top/bottom ferromagnetic layers. Finally in chapter 7, I summarize the MTJ work and present the high quality MTJs with $\mathrm{MgO}$ tunnel barrier made using the unique Bias Target Ion Beam Deposition (BTIBD) system. In chapter 8, I shift gears to focus on another approach to improving the STT performance of MTJs for practical application: the material exploration. a novel magnetic thin film material was developed with perpendicular anisotropy and other unique magnetic and structural properties well suited for developing MTJs with perpendicular magnetic anisotropy. Finally in chapter 9, I summarize the work on MTJs and describe the strategies for future development work on this subject. 


\section{Background theory}

\subsection{Spin polarized transport in magnetic nanostructures}

\subsubsection{Itinerant ferromagnetism}

In magnetic materials like iron, nickel or magnetite, the atomic moments exhibit very strong interactions due to electronic exchange forces which result in a parallel or antiparallel alignment of atomic moments. Such exchange forces are due to the relative orientation of the spins of two electrons by quantum mechanics theory. One of the most successful models in explaining the origin of ferromagnetism is called the itinerant electron model proposed first by E. C. Stoner in 1933 [6]. Basically, it states that the outer electrons in unfilled shells contribute to the magnetic moments of the material. These electrons are not localized and contribute as conduction electrons in the energy band. In the metallic state of $3 \mathrm{~d}$ transition elements, the $3 \mathrm{~d}$ and $4 \mathrm{~s}$ atomic energy levels are broadened into energy bands. In practice, one cannot separate $3 \mathrm{~d}$ and $4 \mathrm{~s}$ orbital clearly since they are strongly hybridized with each other in the solid. Here for simplicity, the two band pictures will still be used and only $3 \mathrm{~d}$ electrons are considered as the itinerant electrons and can carry current through the system.

According to Pauli's exclusion principle, only one electron can occupy a particular state. However, considering the degenerate spin up and spin down states of electrons, two electrons with different spin states could occupy the same state. All energy states are filled up from the ground state up to the highest energy level, which is called the Fermi energy $E_{F}$. The left part of Figure 2.1 shows schematically the density of states (DOS) of a non-magnetic $3 \mathrm{~d}$ metal with equal number of spin up and spin down electrons. It means 
there is no net magnetization, i.e. the spin polarization is zero. Here the spin polarization is defined as

$$
P=\frac{N_{\uparrow}-N_{\downarrow}}{N_{\uparrow}+N_{\downarrow}}
$$

where $\mathrm{N} \uparrow(\mathrm{N} \downarrow)=$ number of electrons with spin up (down). For ferromagnetic metal, as shown in the right side of Figure 2.1, $\mathrm{N} \uparrow$ is larger than $\mathrm{N} \downarrow$, and this leads to a net spin polarization $\mathrm{P}>0$.

When comparing the energy levels of the two different non-magnetic and ferromagnetic states, there are two opposite effects. First, if there is a small imbalance in the number of spin up and spin down electrons, it means there is a transfer of spin down electrons from the spin down band into the spin up band. This leads to more exchange energy in the system, meaning a lowering of the total energy (a gain). On the other hand, a transfer of spin down electrons with energy below the initial Fermi level into the spin up level just above the initial Fermi energy level leads to an increase of the total kinetic energy and thus the total energy (a loss) [7]. Such two competing effects are formulated as the Stoner criterion for magnetism: When

$$
I N\left(E_{F}\right)>1
$$

the system will be ferromagnetic. Here $I$ is the Stoner exchange parameter and $N\left(E_{F}\right)$ is the density of states at the Fermi level. 

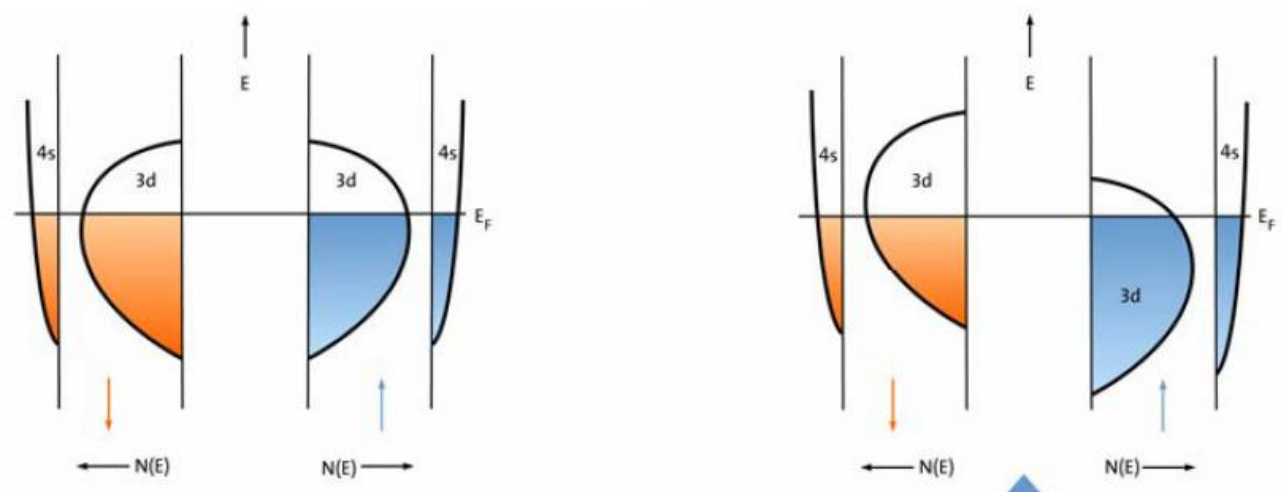

Figure 2.1 (LEFT) a schematic plot is shown for the energy band structure of a d transition metal. The density of states $\mathrm{N}(\mathrm{E})$ is shown separately for the spin up and down electrons and where a simplified separation has been made between the $4 \mathrm{~s}$ and $3 \mathrm{~d}$ band energies. For the non-magnetic state these are identical for the two spins. All energy levels below the Fermi energy are occupied states (orange and blue). The colored area (orange + blue) corresponds to the total number of conduction electrons in the metal. (RIGHT) the corresponding picture is illustrated for a ferromagnetic state, with a spin-polarization chosen to be in the up direction $(\mathbf{N} \uparrow>\mathbf{N} \downarrow$; blue area $>$ orange area). This polarization is indicated by the thick blue arrow at the bottom figure to the right. (Reproduced from Ref. [7])

The right part of Figure 2.1 illustrates the ferromagnetic spin polarization with Stoner's itinerant electron model. There are two important observations that need to be made here. First is the vertical displacement between the spin up and spin down bands due to the exchange energy splitting between the up and down spin densities of states. In this case, more up spin states in blue are filled than the spin down states, so the up and down spin are called the majority spin and minority spin respectively. Secondly, we could see that the density of states at the Fermi level $\mathrm{N}\left(\mathrm{E}_{\mathrm{F}}\right)$ are very different for the two 
spin bands. This would be very important in understanding the spin polarized current and the giant and tunneling magnetoresistance phenomenon in the following sections.

\subsubsection{Spin polarized transport in magnetic nanostructures}

Mott proposed the two-current model in 1936 [8] when he sought an explanation for an unusual behavior of resistance in ferromagnetic metals. He realized that at sufficiently low temperature, where magnon scattering becomes vanishingly small, electrons of majority and minority spin, with magnetic moment parallel and anti-parallel to the magnetization of a ferromagnet, respectively, do not mix in the scattering processes [9]. The conductivity can then be expressed as the sum of two independent and unequal parts for two different spin orientations. This leads to the conclusion that the current in ferromagnets is spin polarized.

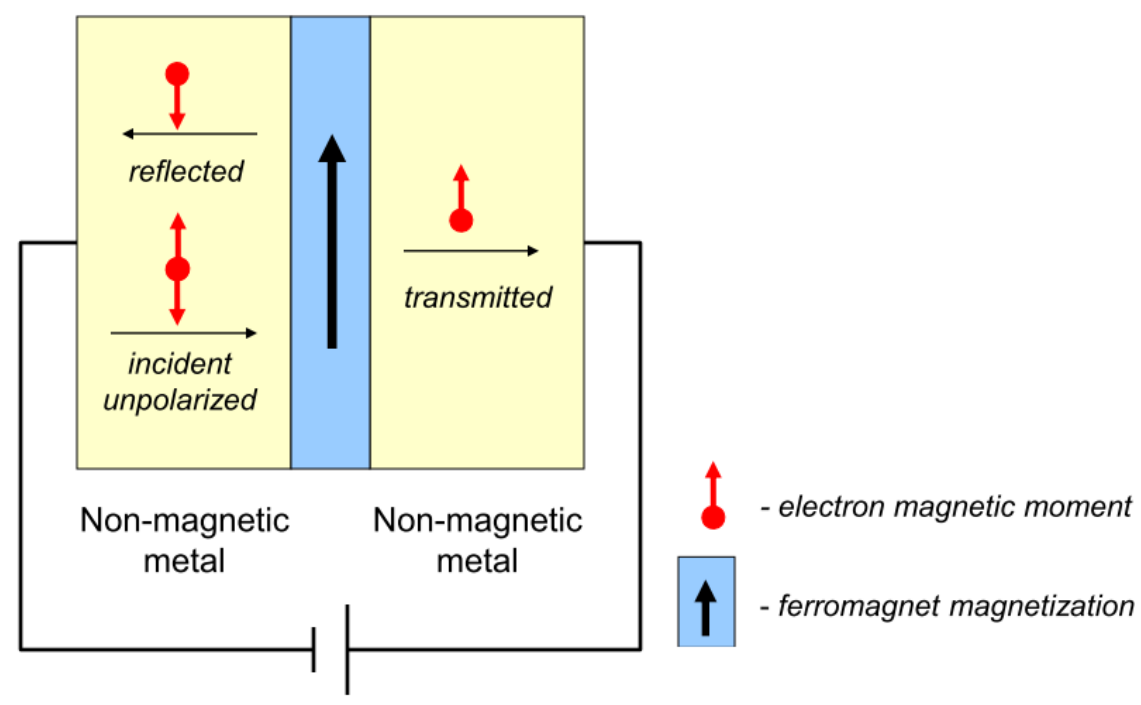

Figure 2.2 Spin polarized electron transport through the Nonmagnetic/Ferromagnetic/Nonmagnetic metal structure. (Adapted from the NRI e-workshop: "Spin Torque Transfer" 08/2009) 
Specifically, as illustrated in Figure 2.2 the electron transport through the nonmagnetic metal/ferromagnet/non-magnetic metal structure, the conductivity is mainly determined by the incident un-polarized 4 s electrons which are easily mobile due to the wide energy range of the bands derived for the 4s-states[8]. Under the two-current mode, we consider the scattering process of the spin up and down electron separately when the un-polarized electrons enter the non-magnetic metal/ferromagnet interface. As I pointed out in the previous section, for ferromagnets with spin up as the majority spin, there are many more spin down than spin up states available at the Fermi level. Therefore the spin down 4 s electrons experience a much stronger scattering at the interface compared with the spin up 4s electrons. As illustrated in Figure 2.2, the spin down electrons are more likely reflected due to the strong scattering while the spin up electrons more likely get transmitted due to weak scattering. In summary, when un-polarized electrons transport through a ferromagnet with its majority spin being up (down), the ferromagnet acts like a spin filter, favoring the spin up(down) current pass through while blocking the spin down (up) electrons via stronger scattering. This serves as the foundation for the giant and tunneling magnetoresistance phenomenon in the next section.

\subsection{Giant and Tunneling magnetoresistance}

\subsubsection{Giant magnetoresistance (GMR)}

The giant magnetoresistance (GMR) was discovered in 1988 in the metallic thin film multilayer structure with alternating ferromagnetic $(\mathrm{Fe})$ and nonmagnetic $(\mathrm{Cr})$ layers $[10$, 11]. The resistance $\left(R_{P}\right)$ of the structure is relatively low when the magnetic orientations 
of the ferromagnetic layers are parallel, while the resistance $\left(\mathrm{R}_{\mathrm{AP}}\right)$ becomes relatively high when the alternating ferromagnetic layers are antiparallel to each other. The magnetoresistance ratio (MR) ratio is defined as the ratio of the change in resistance to the resistance in parallel configuration:

$$
M R=\frac{R_{A p}-R_{P}}{R_{P}}
$$

To understand this GMR phenomenon, we started with the simple three-layer structure that consists of two ferromagnetic layers sandwiched by a nonmagnetic metal layer. This ferromagnetic/nonmagnetic/ferromagnetic (F/N/F) structure is usually called "spin valve". The GMR effect has been observed in both current in the plane (CIP) and current perpendicular to the plane (CPP) configuration. Here I present the explanation of GMR using the CPP configuration which is more relevant to this dissertation. As shown in Figure 2.3, by applying the two-current model [9] mentioned in section 2.1.2, we denote two different resistances to each ferromagnetic layer in two different cases: $\mathrm{R}_{\uparrow}$ when electrons pass through the ferromagnet with the electron spin orientations parallel to its magnetization, and $R_{\downarrow}$ when the spins of the electrons are antiparallel to the ferromagnet magnetization. 
(a)

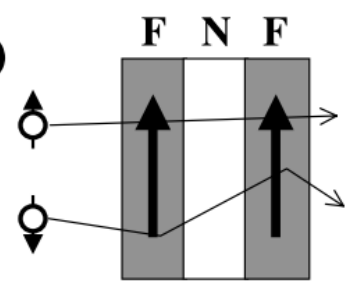

(c)

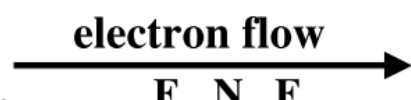

(b)

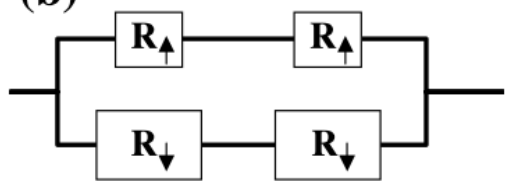

(d)
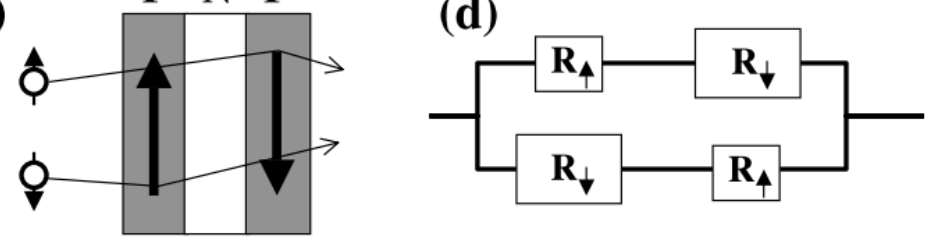
electron flow

Figure 2.3 Two-current mode of the giant magnetoresistance effect in the F/N/F spin valve structure. (a) In the parallel (P) magnetization configuration, the equivalent resistor circuit $(b)$ gives $R_{P}=$ $2 \mathbf{R}_{\uparrow} \mathbf{R}_{\downarrow} /\left(\mathbf{R}_{\uparrow}+\mathbf{R}_{\downarrow}\right)$, while in the antiparallel (AP) configuration (c), the resistor circuit (d) gives $\mathbf{R}_{\mathrm{AP}}=$ $\left(\mathbf{R}_{\uparrow}+\mathbf{R}_{\downarrow}\right) / 2>\mathbf{R}_{\mathbf{P}}$ (Adapted from Ref. [12])

When the magnetizations of the two ferromagnetic layer are parallel $(\mathrm{P})$ as shown in Figure 2.3 (a) and (b), the spin up electrons have a combined resistance $2 \mathrm{R}_{\uparrow}$, and the spin down electrons see a resistance of $2 R_{\downarrow}$, so the total combined resistance in the parallel configuration is $R_{P}=2 R_{\uparrow} R_{\downarrow} /\left(R_{\uparrow}+R_{\downarrow}\right)$. Similarly, in the antiparallel configuration of the two ferromagnets (AP), both spin up and spin down electrons bare the same resistance of $\left(\mathrm{R}_{\uparrow}+\mathrm{R}_{\downarrow}\right)$, so the overall resistance in the AP configuration is $\mathrm{R}_{\mathrm{AP}}=\left(\mathrm{R}_{\uparrow}+\mathrm{R}_{\downarrow}\right) / 2$. When $\mathrm{R}_{\uparrow}$ $<\mathrm{R}_{\downarrow}$, which is the case when you consider the spin-polarized transport discussed in section 2.1.2, we have $R_{A P}$ higher than $R_{P}$, which qualitatively explains the phenomenon of giant magnetoresistance. 


\subsubsection{Tunneling magnetoresistance (TMR)}

Spin dependent tunneling was first discovered by Tedrow and Meservey in a series of experiments in ferromagnet/insulator/superconductor $(\mathrm{F} / \mathrm{I} / \mathrm{S})$ junctions [13-15]. These experiments showed that conduction electrons in ferromagnetic materials are spin polarized and the spin is conserved during the tunneling process. In 1975 Julliere studied the tunneling conductance of ferromagnet/insulator/ferromagnet $(\mathrm{F} / \mathrm{I} / \mathrm{F})$ where $\mathrm{I}$ was an amorphous Ge layer. By applying the same analysis of the tunneling conductance of $\mathrm{F} / \mathrm{I} / \mathrm{S}$ from Tedrow and Meservey $[13,14]$ to $\mathrm{F} / \mathrm{I} / \mathrm{F}$, he formulated a model to describe the change of conductance between the parallel (P) and antiparallel (AP) magnetization of the two ferromagnetic region F1 and F2, as shown in Figure 2.4. The corresponding tunneling magnetoresistance (TMR) in an F/I/F type structure, called a magnetic tunnel junction (MTJ) is defined as

$$
T M R=\frac{\Delta R}{R_{P}}=\frac{R_{A P}-R_{P}}{R_{P}}=\frac{G_{P}-G_{A P}}{G_{A P}}
$$

where the conductance $G$ and resistance $R=1 / G$ are labeled by the relative orientation (P or AP) of the magnetizations of F1 and F2.

According to [16], the conductance in Eq. (2.3) could be expressed as $G_{P} \sim N_{M 1} N_{M 2}+N_{m 1} N_{m 2}$, and $G_{A P} \sim N_{M 1} N_{m 2}+N_{m 1} N_{M 2}$, where $N_{M i}$ and $N_{m i}$ are the spin-resolved density of states for majority and minority spin in the ferromagnets $F_{i=1,2}$. Recalling the definition of spin polarization $P_{i}=\left(N_{M i}-N_{m i}\right) /\left(N_{M i}+N_{m i}\right)$ stated in Eq. (2.1), the Eq. (2.3) could be rewritten as

$$
T M R=\frac{2 P_{1} P_{2}}{1-P_{1} P_{2}}
$$


(a)
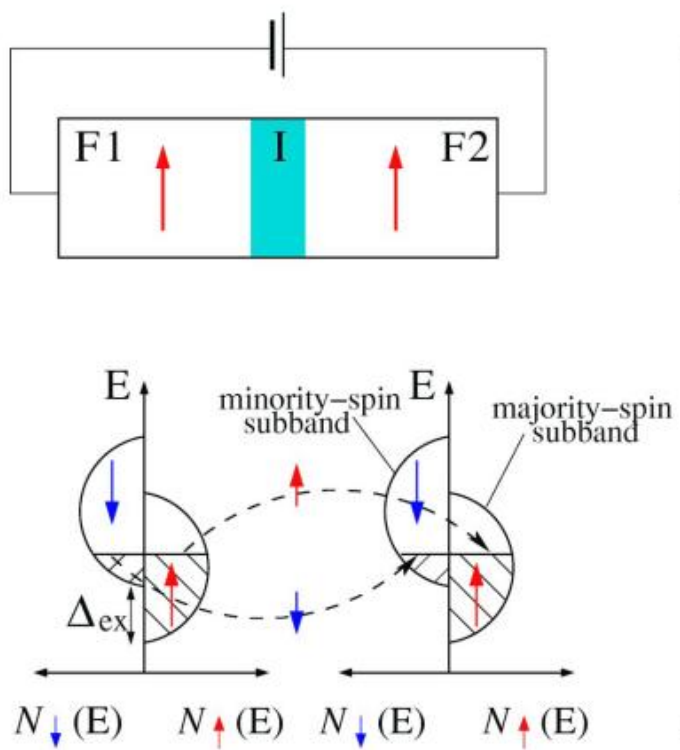

(b)
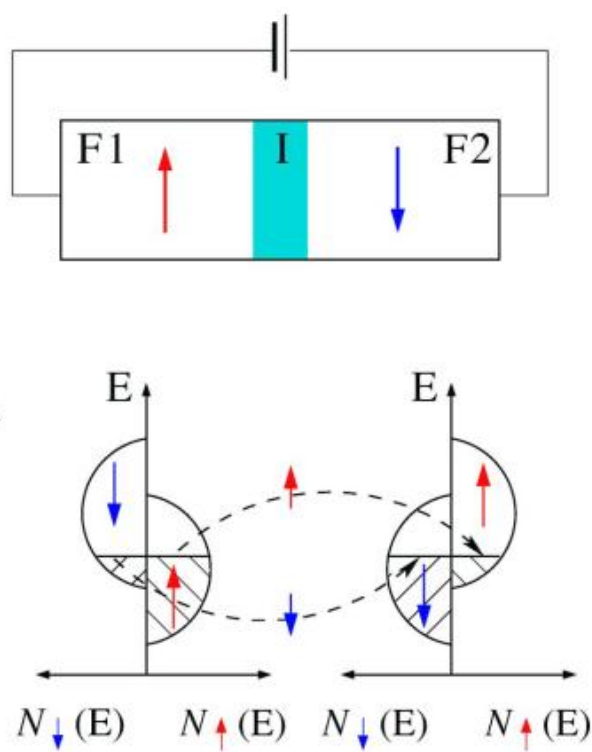

Figure 2.4 Schematics of electron tunneling in ferromagnet/insulator/ferromagnet (F/I/F) tunnel junctions: (a) Parallel and (b) antiparallel orientation of magnetizations with the corresponding spin-resolved density of the $\mathbf{d}$ states in ferromagnetic metals that have exchange spin splitting $\Delta_{\mathrm{ex}}$. Arrows in the two ferromagnetic regions are determined by the majority-spin sub-band. Dashed lines depict spin-conserved tunneling. (Reproduced from Ref. [9])

\subsection{Magnetic tunnel junctions (MTJs)}

As introduced in section 2.2.2, the trilayer junction consisting of two ferromagnetic layers sandwiching an insulating barrier is called a magnetic tunnel junction (MTJ). The first experiment of spin-dependent tunneling in the MTJ type structure $\mathrm{Fe} / \mathrm{Ge} / \mathrm{Fe}$ by Julliere in 1975 [17] was carried out at low temperature and an TMR effect of $14 \%$ was reported. This type of work was continued by Maekawa and Gafvert in 1982 [16] on $\mathrm{Ni} / \mathrm{NiO} / \mathrm{FM}$ system where FM stands for $\mathrm{Fe}$, Co or Ni. They were only able to observe a 
few percent of TMR in low temperature as well. The breakthrough came in 1995 when two groups, one from MIT [2] and the other from Japan [1] reported significant progress in making MTJs showing a room temperature TMR effect. Moodera and his group in MIT measured the MTJ structure of $\mathrm{CoFe} / \mathrm{Al}_{2} \mathrm{O}_{3} / \mathrm{Co}$ (or NiFe) and got TMR value of $24 \%$ at $4.2 \mathrm{~K}$ and $12 \%$ at room temperature. Similarly, Miyazaki and his collaborator obtained $30 \%$ TMR at $4.2 \mathrm{~K}$ and a room temperature TMR of $18 \%$ using $\mathrm{Fe} / \mathrm{Al}_{2} \mathrm{O}_{3} / \mathrm{Fe}$ MTJs.

The achievement of room temperature TMR in 1995 attracted a great deal of interest from research groups across the world, mainly due to the huge potential industrial applications of MTJs and the broader research field called "Spintronics" by exploring another degree of freedom of electron, the spins of the electron, besides the conventional manipulation of the charges of electron [18]. Thousands of papers have been published on the subject of MTJs after the initial breakthrough in 1995 in order to improve the TRM performance of MTJs for industrial applications, among which the two major applications are using MTJs as the hard disk drive (HDD) recording head sensors and non-volatile magnetic random access memory (MRAM) cells.

The initial breakthrough on MTJs with simple electrodes like CoFe and Fe produced TMR just over $10 \%$. Materials wise, by replacing polycrystalline electrodes like CoFe and $\mathrm{NiCoFe}$ with amorphous $\mathrm{CoFeB}$ and optimizing the growth condition, the record high MR value of $70 \%$ was achieved on MTJs with aluminum oxide barrier [19]. However, this is still not high enough for many spintronic device applications. The breakthrough came when first principle calculations predicted that MTJs with epitaxial $\mathrm{Fe} / \mathrm{MgO} / \mathrm{Fe}$ structure could provide significant spin filtering that would enhance the MR 
to over $1000 \%$ [20], and was soon demonstrated experimentally with TMR around $200 \%$ by two different groups independently [21, 22]. Combing the $\mathrm{CoFeB}$ electrode and $\mathrm{MgO}$ tunnel barrier, a MR value of $230 \%$ at room temperature was achieved in MTJs with a $\mathrm{CoFeB} / \mathrm{MgO} / \mathrm{CoFeB}$ structure [23]. By optimizing the composition of $\mathrm{CoFeB}$, recently TMR ratios of up to $500 \%$ have been achieved at room temperature (RT) in $\mathrm{CoFeB} / \mathrm{MgO} / \mathrm{CoFeB}$ MTJs [24]. All of these efforts have greatly enhanced the potential of MTJs in spintronic device applications like data storage, magnetic sensors, etc.

Besides the improvement of materials for making better MTJs, different layered structures have also been intensively studied to produce MTJs more suitable for practical applications. As shown in Figure 2.5, the initial MTJs have the simplest structure of two ferromagnetic layers sandwiched by an ultrathin insulating barrier layer. One of the ferromagnetic layers has relatively large magnetic anisotropy and its magnetization direction is fixed as the reference orientation, so this ferromagnetic layer is called the reference layer (RL) or fixed layer. The other ferromagnetic layer, called the free layer (FL), has relatively low coercivity and hence its magnetization could be flipped easily into one of the two easy axis directions, to achieve the relative high and low resistance states for the whole MTJ stack.

(a)

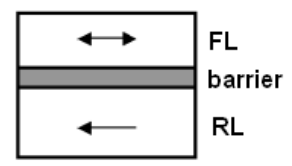

(b)

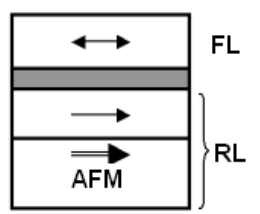

(c)

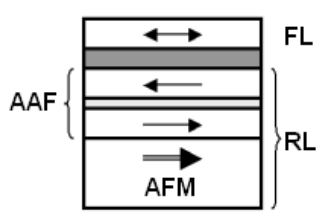

(d)

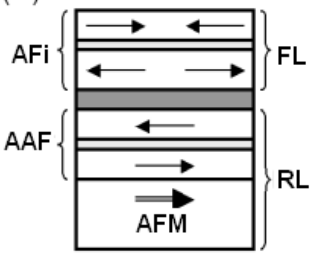

Figure 2.5 The evolution of MTJ structures: (a) MJTs with single ferromagnetic free layer (FL) whose magnetization could be rotated to achieve high and low resistance state, and the reference 
layer (RL) whose magnetization is fixed. (b) MTJs with exchange biased RL. (c) Exchange biased artificial antiferromagnet (AAF) as the RL. (d) Exchange biased AAF as the RL and artificial ferromagnet (AFi) as the FL of MTJs. (Adapted from Ref. [25])

To achieve a more stable reference layer, the exchange bias (EB) mechanism was utilized by adding an antiferromagnetic layer (AFM) next to the ferromagnetic (FM) reference layer to form the bilayer exchange bias system. Basically, due to the exchange bias coupling between the FM/AFM layers, the hysteresis loop of the EB system will be shifted off center along the field direction caused by the EB anisotropy [26]. The details of the EB system will be discussed in chapter 4 . Here by shifting the hysteresis loop of the RL, a more stable and wider range separation of the two ferromagnetic layers was achieved. To reduce the stray magnetic field from the RL and further stabilize the RL, the ferromagnetic layer in the EB system was replace by a trilayer system with two ferromagnetic layers sandwiched by a nonmagnetic metal layer through the antiferromagnetic coupling as shown in Figure 2.5(c). This sandwich is called an Artificial Antiferromagnet (AAF) or Artificial Ferrimagnet (AFi), depending on their net magnetic moment [25], or more commonly called Synthetic Antiferromagnet (SAF). Later, such sandwiched system by antiferromagnetic coupling was also used as the FL of MTJs with the main purpose of reducing the stray field [27]. 


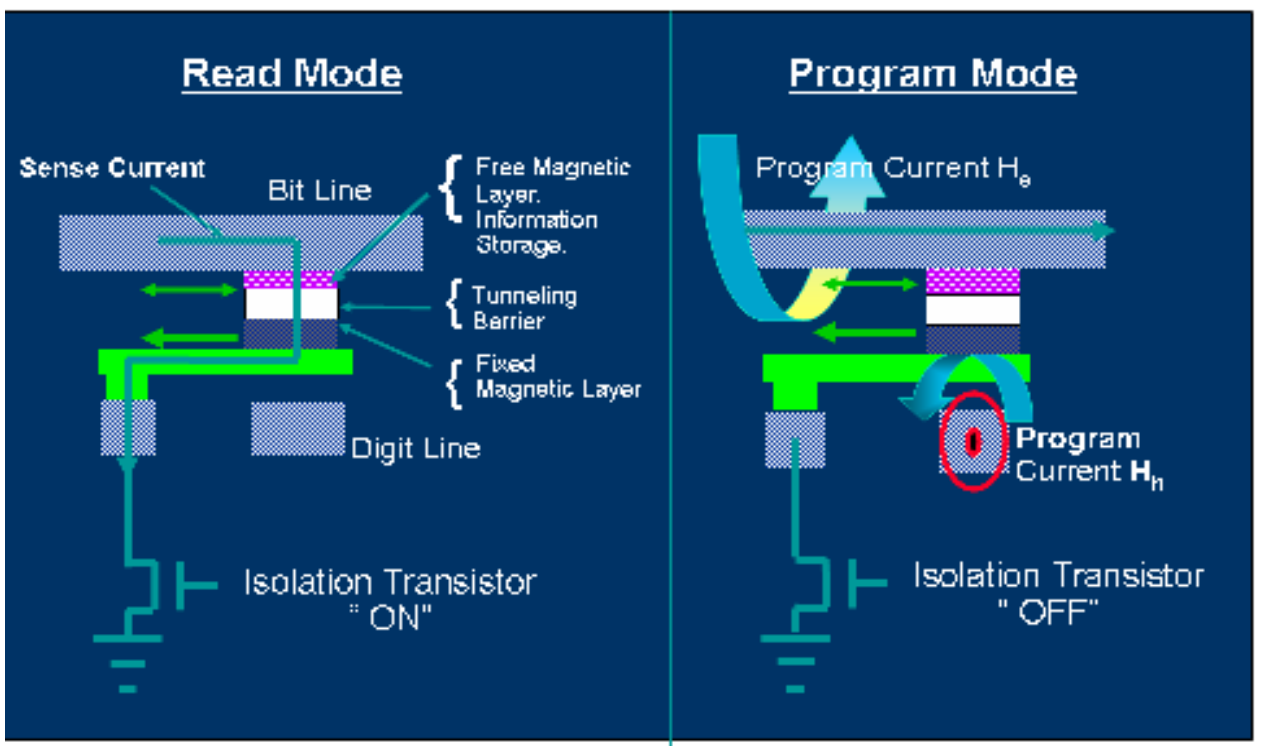

Figure 2.6 Read and program mode of a MRAM unit cell with 1Transistor-1MTJ architecture. In the read mode, the transistor is turned on, and a sensing current is passing through the bit line to the read out the resistance state of the MTJ; In the program mode, the transistor is turned off, a program current passed through the same bit line as well as the digit line to generate magnetic field to switch the orientation of the free layer of MTJ in order to change its resistance state. (Adapted from Spintronics lecture notes, Prof. Stu Wolf, UVA 2006)

As we mentioned earlier in this section, one of the major industrial applications of MTJs is the Magnetic Random Access Memory (MRAM), where a MTJ is used as the unit cell and the relative high and low resistance states are used to store the " 0 " and " 1 " bit information. As shown in Figure 2.6 is the 1T(Transistor)-1MTJ architecture MRAM, where a transistor is used to control the open and closed states of the circuit during the read and write (program) process, and the external field is used to switch the magnetic orientation of the FL of the MTJs. In the read mode, the transistor is turned on, and a sensing current is passing through the bit line to read out the resistance state of the MTJ; In the program mode, the transistor is turned off, and a program current passed through 
the same bit line as well as the digit line, which sits orthogonally to the bit line, to generate the external magnetic field to switch the orientation of the free layer of MTJ in order to change its resistance state.

The non-volatile MRAM technology using MTJs as a storage unit in principle have advantages over all existing memory technologies like DRAM, SRAM and FLASH as we mentioned in chapter 1. The development of toggle switching in MRAM chip systems [3], an innovative method of switching the MTJs cell between high and low resistance states, paved the way for the swift transition of this single device to a fully engineered storage system. Conventional MRAM chips with a toggle switching scheme has been commercialized for several years with good market feedback. However, there is an inherent problem of scalability associated with the current generated external field switching scheme of the MRAM architecture. In order to maintain the thermal stability of the patterned MTJ recording bit, i.e. keep the magnetization orientation of the MTJ free layer from being randomly switched due to thermal fluctuation, for an industrial retention time of 10 years, the following criteria should be met:

$$
\frac{K_{u} V}{k_{B} T} \sim 60
$$

where $K_{u}$ is the magnetic anisotropy, $V$ is the volume, $k_{B}$ is the Boltzmann constant and $T$ is the temperature. When scaling down the MTJ cells for higher density MRAM, i.e. decreasing the volume of the MTJ cells, the $K_{u}$ of the MTJ free layer material must increase in order to meet the same thermal stability. Higher $K_{u}$ means it requires higher external filed to switch the free layer, hence the current used to generate the magnetic field needs to be increased as well. So the required current is inversely related to the bit size and becomes too large to be practical as the MRAM cell scales. Also the complicated 
structure associated with the toggle switching stands as another bottle neck in the way of getting higher density MRAM.

\subsection{Spin torque transfer (STT)}

Fortunately, a new method of switching called Spin Torque Transfer (STT) provides a promising solution to this scalability problem. It was predicted in 1996 by two different groups $[4,5]$ that spin polarized current could exert a spin torque on the magnetic moments when passing through a ferromagnetic layer, generating spin waves or even completely switching the magnetization orientation of the ferromagnetic layer if properly engineered. This prediction was based on a generally accepted argument: for a system consisting of itinerant electrons and local moments, the total angular momentum is conserved even when the system is out of equilibrium [28]. The STT mechanism is illustrated in Figure 2.7 within a spin valve or MTJ structure frame.

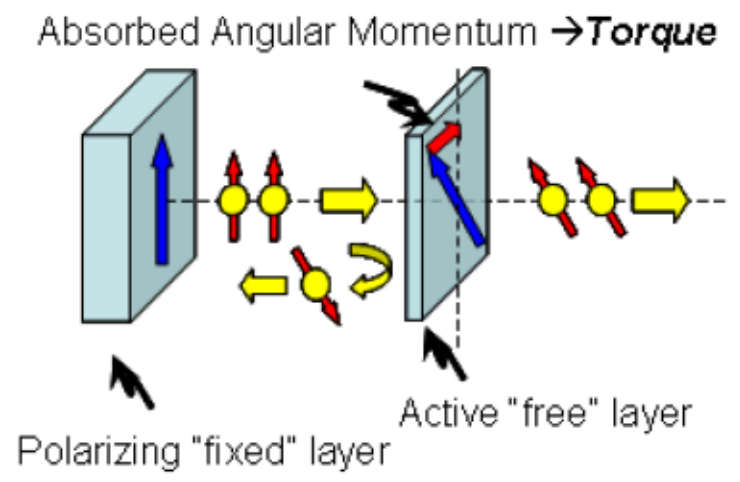

Figure 2.7 Spin Torque Transfer (STT) inside a spin valve or magnetic tunnel junction scheme. When an electron current passes through the ferromagnetic fixed layer, it becomes spin polarized and when flowing through the second ferromagnetic free layer, it exerts a spin torque on the 
magnetic moment of the free layer due to the interaction between the electron spin and local magnetization. (Adapted from Spintronics lecture notes, Prof. Stu Wolf, UVA 2006)

Compared with the use of an external field generated by passing a current in the conventional toggle switch scheme, the STT switching scheme has the correct scaling behavior, namely, it is the current density rather than the absolute current that is important, so the actual current scales down properly with the device size. The theoretical expression of this critical current density required to switch the magnetization of a ferromagnet is predicted by Slonczewski [5]:

$$
J_{c}=\frac{2 e \alpha M_{S} t_{F}\left(H_{K} \pm H_{e x t}+2 \pi M_{S}\right)}{\hbar \eta}
$$

where $e$ is the electron charge, $\alpha$ is the damping constant, $M_{S}$ is the saturation magnetization , $t_{F}$ is the thickness of the free layer, $H_{K}$ is the effective anisotropy field including magnetocrystalline anisotropy and shape anisotropy, $H_{\text {ext }}$ is the external field, $\hbar$ is the reduced Planck's constant, and $\eta$ is the spin transfer efficiency. The " \pm " sign denotes the positive and negative direction of the electron current flow, with the positive direction defined as the electrons flow from the fixed reference layer to the free layer.

The STT switching effect was initially demonstrated in metallic spin valve nanopillars [29-31], showing MR values between $0.5 \sim 5 \%$ and the critical current density $J_{c}$ ranging from $5 \times 10^{6}$ to $10^{8} \mathrm{~A} / \mathrm{cm}^{2}$ depending on the structure. Later the focus was shifted to the study of STT switching of MTJs which is closer to practical applications due to their much larger TMR values, as a high TMR value is needed for fast read operation in the MRAM application. The first experimental demonstration of STT switching of an MTJ was achieved by a group from Grandis Inc [32]. They were able to 
get $42 \%$ TMR and the resistance-area product (RA) value $\sim 40 \Omega \mu^{2}$. The critical current density they obtained was around $6 \times 10^{6} \mathrm{~A} / \mathrm{cm}^{2}$, but this value is still considered too high to be practical. In order for this current drive STT switching to be viable in the MRAM architecture, the critical current density needs to be reduced to the level $\sim 10^{5}$ $\mathrm{A} / \mathrm{cm}^{2}$ for a few reasons. First of all, the switching current in the MRAM architecture is delivered by a CMOS transistor, which typically supplies $100 \mu \mathrm{A}$ per $100 \mathrm{~nm}$ gate width. In order to achieve higher density MRAM with a 1T-1MTJ architecture, the size of the transistor needs to be reduced, resulting in smaller current output from the transistor and hence a smaller switching current density becomes inevitable. Also, large current density will produce a large voltage potential across the device, increasing the probability of material fatigue and tunnel barrier breakdown, compromising the endurance and reliability of the overall device performance [33].

With materials engineering, as we can see from eq. 2.6, there are several key parameters that could be optimized in order to reduce the critical current required for STT switching. By using materials with a low saturation magnetization $M_{S}$ and/or a low damping parameter $\alpha$ as well as a high spin transfer efficiency, we would expect a reduced critical current $J_{c}$, which is essential for the successful transformation of the technology of STT switching of MTJs into the next-generation MRAM called Spintorque transfer-random access memory (STT-RAM). One good example in terms of materials engineering was the introduction of $\mathrm{CoFeB}$ as the new ferromagnetic electrode of MTJs, due to its advantages of low $M_{S}$ compared with conventional materials like Co, $\mathrm{CoFe}$ and $\mathrm{NiCoFe}$, etc. For a typical $\mathrm{CoFe}_{10}$, the $M_{S}$ value is $1540 \mathrm{emu} / \mathrm{cc}$, whereas the $M_{S}$ of $\mathrm{Co} 40 \mathrm{Fe}_{20} \mathrm{~B}_{20}$ is less than $1050 \mathrm{emu} / \mathrm{cc}$ [28], depending on the layer thickness. A 
rough estimate from eq. 2.6 shows a reduction in $J_{c}$ almost by half, assuming other parameters unchanged. On the other hand, by replacing the amorphous $\mathrm{AlO}_{\mathrm{x}}$ barrier with crystalline $\mathrm{MgO}$, the TMR increases from $30-70 \%$ to $300 \%$ [21, 22] at room temperature, and the $J_{c}$ is expected to be lowered by about two times due to much higher spin polarization and spin dependent tunneling [28].

Despite the intensive research activities around the world in the past years, lowering the critical current density of STT-RAM to a practical level and making the device small enough to compete with other technologies like much higher density FLASH memory, remain the key ongoing challenges of developing next generation STT-RAM. To further improve the MTJ performance for STT-RAM application, the research focus of MTJs has been shifting recently from MTJs consisting of magnetic layers with in-plane magnetization such as $\mathrm{Fe}, \mathrm{CoFe}$ or $\mathrm{CoFeB}$ [21-23] to materials with out-of-plane perpendicular magnetic anisotropy (PMA), for example, the rare-earth transition metal (TbFeCo, GdFeCo, etc. [34]) and tetragonal intermetallic compounds ( $\mathrm{L}_{0}$ phase FePd, CoPt, etc. [35]). When MTJs are patterned into submicron size with an aspect ratio of one, the in-plane magnetization becomes unstable, and generally an aspect ratio of at least two is needed to maintain the thermal stability [36]. On the other hand, ferromagnetic materials with PMA will be thermally stable even with an aspect ratio of one. Also, as discussed in eq. $\mathbf{2 . 5}$, when the size of a MTJ memory cell is reduced, much larger magnetic anisotropy is needed to maintain the thermal stability. Generally, materials with PMA have larger anisotropy compared with conventional in-plane magnetic materials like $\mathrm{CeFe}$ or $\mathrm{CoFeB}$. Also it was theoretically predicted that perpendicular magnetic devices have the potential to achieve a switching current lower 
than that of in-plane magnetic devices with the magnetic anisotropy field [37]. Therefore, the perpendicular MTJ (p-MTJ) structure has the advantages of achieving a low switching current and larger thermal stability, and is suitable for integrating STT-RAM elements with higher packing density. In this dissertation, $\tau$-phase $\mathrm{MnAl}$, another tetragonal intermetallic compound having the similar $\mathrm{L}_{0}$ structure and PMA as FePd and CoPt, will be extensively studied regarding its synthesis methods, annealing treatments as well as its magnetic and structural characterization, with the purpose of optimizing the properties of $\tau$-MnAl as the electrode for p-MTJs with low switching current and high packing density for next generation STT-RAM application. 


\section{Experimental techniques and sample fabrication}

\subsection{Ion beam deposition tools}

\subsubsection{Biased target ion beam deposition (BTIBD)}

Among the many techniques to grow magnetic tunnel junction (MTJ) structures, ion beam deposition (IBD) has the advantages of low processing pressures, directional sputtered flux, high adatom flux energy, low sputtering rate and independent control of target and substrate environment [38]. However, the conventional IBD methods were not designed for optimal deposition conditions for MTJ multilayer structures. For example, the conventional IBD can only be operated using relatively high sputtering ion energies and relatively low pressure with no substantial background gas scattering [39]. This is because it is harder to focus the sputtering ion beam at low energies, meaning a larger fraction of the ion beam could miss the targets and sputter off undesired materials from the vacuum system hardware, causing overspill contamination [38]. Using higher acceleration voltages better focuses the ion beam on the target. Unfortunately, higher accelerating voltage gives the adatoms higher kinetic energies when they reach the substrate, causing intermixing problems at the interfaces, which is detrimental to the tunneling magnetoresistance (TMR) performance. Low energy IBD would improve the uniformity of the film thickness and reduce interlayer mixing but requires a large target to avoid overspill sputtering. Previous simulation studies [40-42] have shown that multilayer structures grown by sputtering processes with adatom kinetic energies within the range of 1-5 eV would have good surface flatness while interlayer mixing remains relatively low. 
The biased target ion beam deposition (BTIBD) technique was developed [38, 43] to overcome some problems of the conventional IBD method. The BTIBD system consists of three vacuum chambers: i) the cassette chamber (loading dock) which contains a cassette that can hold up to 12 pieces of 8 inch wafers; ii) the transport chamber, equipped with a robotic arm for transferring wafers from cassette chamber to the process chamber; and iii) the processing chamber, where the targets, stage and ion beam guns are installed and where the sputtering process takes place. The schematic illustration of the BTIBD processing chamber is shown in Figure 3.1. The targets assembly holds up to six metal targets in a hexagonal fashion, so up to six different materials could be used when depositing multilayer film stacks. The stage is located on the left side of the process chamber facing the target assembly. The robotic arm transfers the wafer into the processing chamber where it is clamped to the stage ready for deposition. The stage can be titled to vary the incident angle of the sputtered species from $0^{\circ}$ to $90^{\circ}$ (normal incidence). Also the stage can be rotating during the deposition to enhance the film uniformity across the wafer. It is also possible to apply a magnetic field along a desired direction in the plane of the wafer in order to induce magnetic easy axis during growth. 


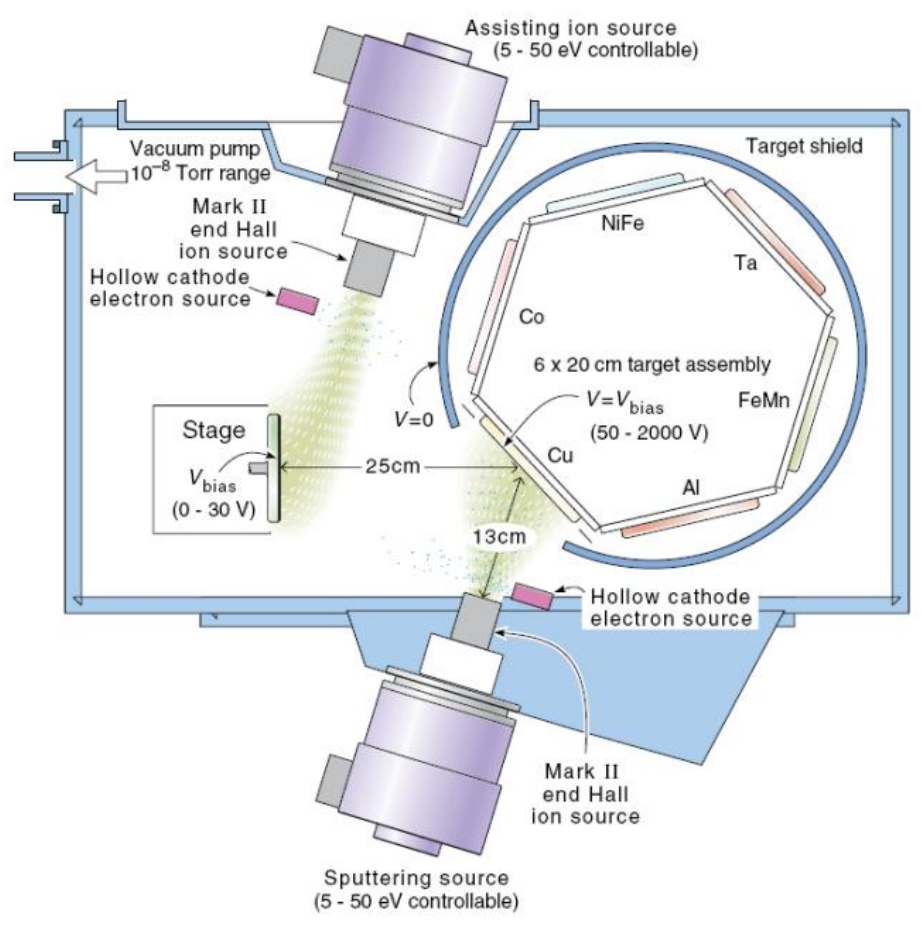

Figure 3.1 Schematic illustration of the process chamber of the BTIBD system (top view, adapted from Ref. [39])

Instead of using a conventional grid ion beam source, the BTIBD applies two novel low energy ion sources that combine an end-Hall ion source [44] and a hollow cathode electron source [45]. The advantage of this combined source over conventional IBD source is that it can provide a very high density of inert gas ions with very low energy (several eV) [39]. As shown in Figure 3.1, two identical ion sources are installed in the process chamber. One is constructed facing the target assembly and is used to provide low energy $(0-50 \mathrm{eV})$, high density ions in front of the selected sputtering target. This is the main ion source gun. The other is installed on the top of the chamber facing down directly to the stage surface when it is not tilted. This assisting ion source could be used to produce low energy $(0-50 \mathrm{eV})$ ions for the ion beam assisted deposition, or used for 
ion beam etching of samples when providing higher energy ions $(50-100 \mathrm{eV})$. In the sputtering process, when a high negative bias voltage (50-2000 V) is applied to the target, the low energy inert gas ions from the main ion source will strike the target surface at a near-normal incident angle due to the high voltage potential difference. By controlling the negative bias voltage, the energy of the incident inert ions can easily be controlled. Control of the kinetic energy of the species sputtered from the target surface is possible and allows for the deposition rate to be tuned.

Another advantage of using the low energy inert ions is near elimination of the overspill contamination problem faced by the conventional IBD system. Most of the inert ions will hit the target surface due to the large voltage potential difference between the selected target and the low energy ions, while only a small portion could miss the target. Since all the system components are grounded (chamber wall, shutter, shielding, etc.), any ion missing the target will produce negligible sputtered material due to the low energy and lack of accelerating potential. Such a design allows for the use of an ion beam broader than the target, and result in a uniformly illuminated target. Uniform illumination provides improved target material utilization efficiency and deposition thickness uniformity. The high ion density resulting from the BTIBD system design, can compensate for the loss of deposition rate due to low sputtering yield with low energy ions. The low sputtering energy leads to low kinetic energy of the species sputtered from the target material, which is beneficial to the interfacial smoothness and the reduction of interlayer diffusion mixing.

Another advantage of the BTIBD ion source is the independence of ion trajectories from the source to the target with respect to the bias voltage applied to the target. A 
plasma sheath develops at the surface of the target when it is negatively biased. Due to the small thickness of the sheath $(\sim 2 \mathrm{~mm})$ compared with the distance between the ion source and the target $(130 \mathrm{~nm})$, the target bias has no substantial effect on the ion trajectories [39]. In addition, the space between the target shield and target edge is smaller than the sheath thickness, so the plasma penetration at the target edge is effectively prevented. Such design allows for the stable operation of the ion source at a wide range of target voltages $(\sim 100$ to $>100 \mathrm{eV})$ and target currents $(0-500 \mathrm{~mA})$. Precise control of target voltage facilitates atomic level modulation of the intralayer and interlayer profiles during deposition and optimization of the multilayer structure properties. The detailed analysis of the bias voltage effect and interface engineering will be discussed in chapter $\mathbf{6}$. The BTIBD ion sources are designed to be capable of operating over a broader range of processing pressure $\left(10^{-4}\right.$ to $5 \times 10^{-3}$ Torr $)$, allowing some control of the background gas scattering of the inert ions before they reach the substrate. Overall, the BTIBD provides a way of controlling the adatom energies over a very wide range (from several $\mathrm{eV}$ to several tens $\mathrm{eV}$ ) [39].

The key component of the this novel BTIBD system is the low energy broad beam ion source, and it is the combination of a Mark $\mathrm{II}^{\mathrm{TM}}$ end-Hall ion source and and HCES 5000 hollow cathode electron source as shown in Figure 3.2. The Mark II end-Hall ion source acts as the anode ionizing the inert Ar gas fed into the source. It is made with hard and soft magnetic materials and a gas inlet. The hollow cathode consists of a narrow tube, a "keeper" surrounding the cathode, and an emission end. The hollow cathode generates the electrons that flow toward to the anode of the end-Hall source where the ions are created. The electric and magnetic fields near the anode determine both ion and electron 
path lengths, control the inonization in the discharge, as well as accelerate the ions out of the anode [39]. The most essentail advantage of this ion source is that it can provide inert ions with very low energy and rather broader energy range. This feature is in sharp contrast to the conventional IBD system equipped with grid ion beam source where ions only narrowly distributed in a high energy range are allowed in order to focus the ion beam onto the target to reduce overspill contamination. An advantage of the end-Hall source is that the anode current can be controlled independently regardless of the ion beam details, so the number of emitted ions can be independently optimized to achieve the best deposition conditions [39].

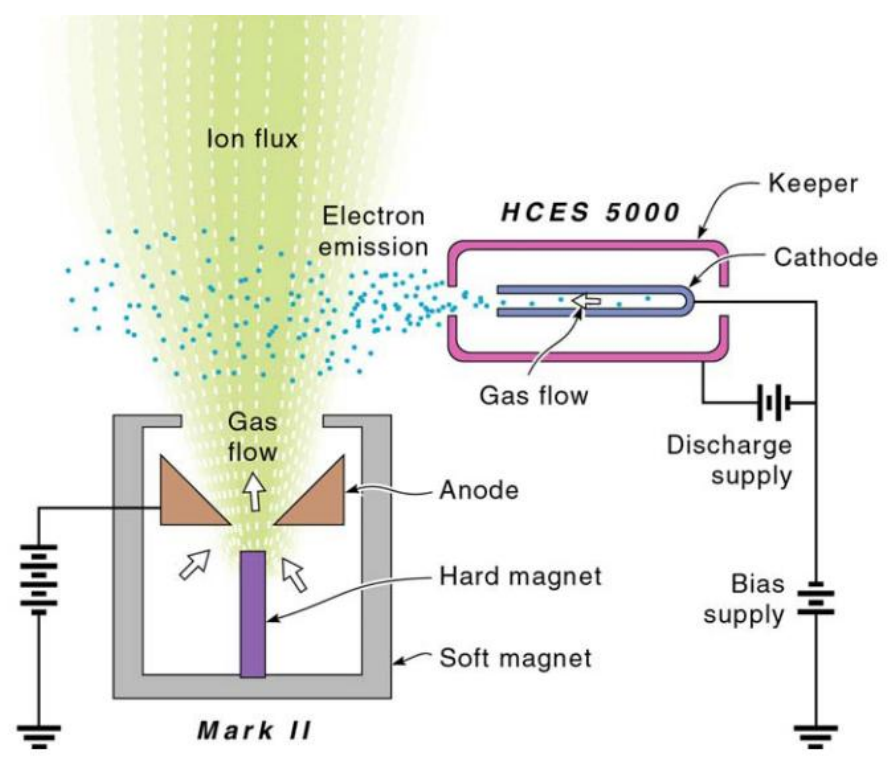

Figure 3.2 Schematic layout of Mark II End-Hall ion source and HCES 5000 Hollow Cathode electron source. (Adapted from Ref. [39]) 


\subsubsection{Reactive biased target ion beam deposition (RBTIBD)}

The BTIBD system, co-developed by the University of Virginia and 4Wave Inc., was designed and optimized for thin films and multilayer structure deposition with metal targets allowing for precise control of the intralayer and interface profile engineering. However, the BTIBD is limited to the deposition of metallic layers and only one target can be selected at a time for the deposition. Also it lacks an important function for material exploration in that the stage cannot be heated for growth at elevated temperature. A second deposition tool was developed to address the limitations of the BTIBD system. The modified tool called Reactive Bias Target Ion Beam Deposition (RBTIBD), was developed by the same company 4 Wave Inc. to focused on the material exploration including both oxides and metallic compounds.

The RBTIBD system is equipped with the same low energy ion source that combines a Mark II $^{\mathrm{TM}}$ end-Hall ion source and a hollow cathode electron source (HCES) like the BTIBD system. It was designed as a compact version of the BTIBD so there are only two chambers, one load lock chamber for mounting sample substrates without breaking the vacuum of the main processing chamber, and the other is the processing chamber where the targets and a single ion source are located. A mechanical pump is used to pump down the load lock down to about $75 \mathrm{mT}$ before opening the isolation gate valve between the two chambers. A cryogenic pump is installed underneath the main process chamber and high vacuum $\left(2.0 \times 10^{-8}\right.$ Torr $)$ state can be achieved due to the compact design of the system. The schematic illustration of the RBTIBD processing chamber is shown in Figure 3.3. Six $100 \mathrm{~mm}$ diameter metallic targets sit in a hexagonal housing facing each other. The ion source and rotatable $100 \mathrm{~mm}$ diameter sample stage lie along the center 
axis on either side of the target housing. A two stage target shutter carousel (not shown in Figure 3.3) simultaneously exposes three targets, while blocking the remaining target slots.

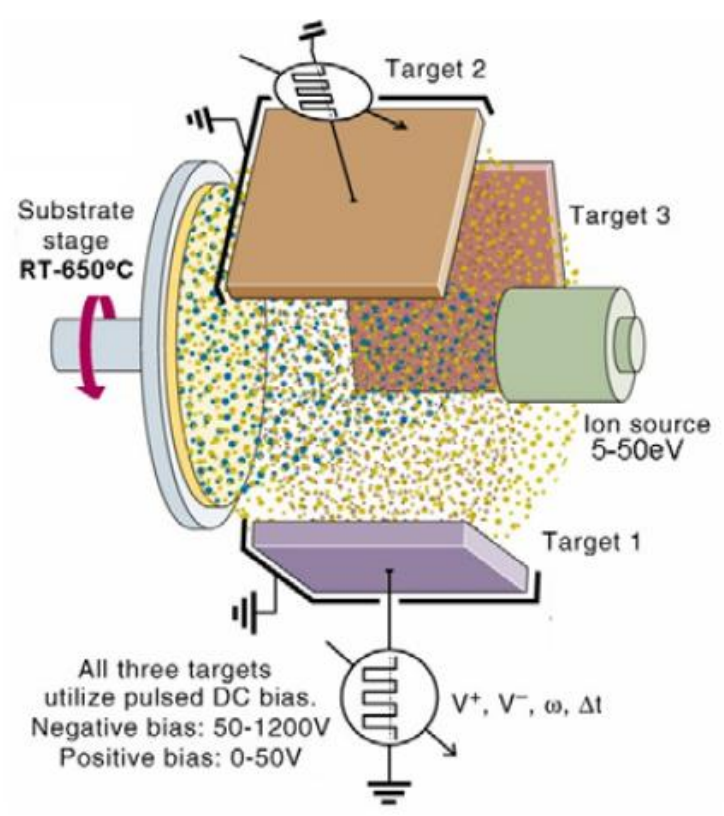

Figure 3.3 The schematic illustration of the RBTIBD processing chamber. (Adapted from Ref. [33])

Ultra high purity (UHP) Ar gas (99.999 \%) is used by the ion source to generate the ion beam plasma. There are three gas lines connected to the ion source in the chamber. Two of them are Ar gas lines, one through the anode and the other through the cathode. The third gas line provides gas, mostly $\mathrm{O}_{2}$ or $\mathrm{Ar} / \mathrm{O}_{2}$ mixture, to be used for reactive sputtering to create oxides materials. The gas flow rates are controlled by $\mathrm{MKS}^{\mathrm{TM}}$ digital mass flow controllers (MFC). Like in the BTIBD system, the ion source operates in a rather wide range of processing pressure $\left(10^{-4}\right.$ to $5 \times 10^{-3}$ Torr), allowing some control of the background gas scattering of the inert ions before they reach the substrate. 


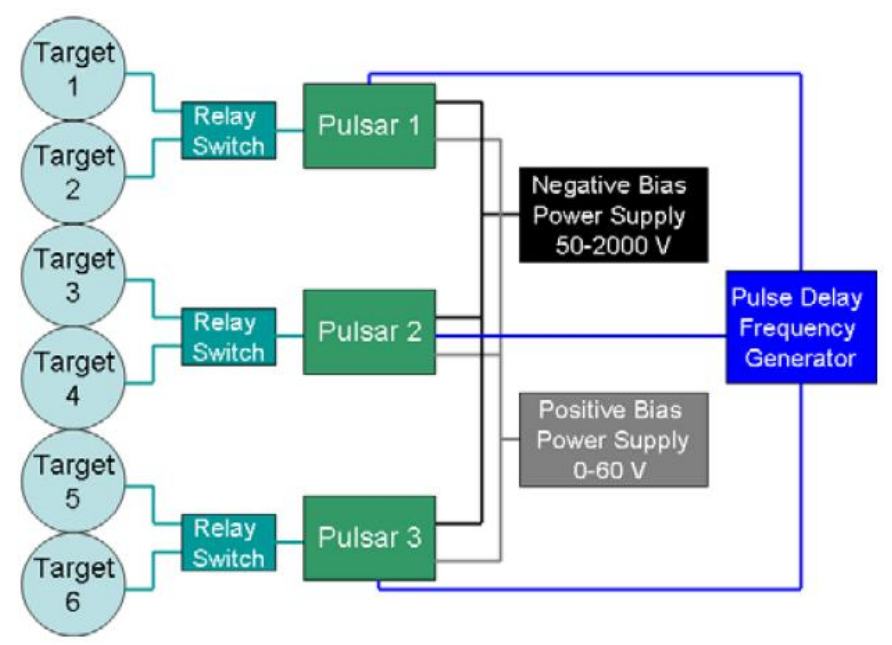

Figure 3.4 The schematic illustration of the pulsed dc bias control. (Adapted from Ref. [33])

Different from the BTIBD system, the targets in RBTIBD system are pulsed dc biased, i.e. the targets are pulsed alternatively between a large negative bias voltage and a small positive bias voltage at a controlled frequency and pulse period. As we discussed in section 3.1.1, sputtering only occurs when negative bias voltage is applied, and no sputtering takes place during the positive biased period. The power supply for positive bias provides the voltage and current at the range of 0-60 V and 0-5 $\mathrm{A}$, while the ranges from the negative power supply are 0-2 kV and 0-1 A. As shown in Figure 3.4, two targets are connected in parallel to a relay switch, which is used to control which of the two targets is connected to the pulsar in series. All pulsars are connected to both a negative and positive bias power supply. A Berkley Nucleonics model 565 pulse delay frequency generator is used to program the frequency and pulse period of the three pulsars in separated channels. Up to three targets that are not controlled by the same relay 
could be pulsed dc biased simultaneously with an independent frequency and pulse period of its own in such configuration [33]. Considering the fact that the sputtering process only occurs when the target is negatively biased, such configuration allows for rapid exploration of a wide range of phase space in alloy material system by systematic modulation of the relative frequency and (or) negative pulse period of each selected target material during the combinatorial sputtering process, as we know that the negative pulse period is equivalent to the total pulse period (1/frequency) minus negative pulse period.

The advantage of using pulsed dc bias in the RBTIBD compared with simple dc bias used in the BTIBD system becomes essential during the reactive sputtering of oxides materials when oxygen is introduced into the processing chamber. $\mathrm{O}_{2}$ ions are reactive and can easily bond chemically to the metallic target material surface. This could cause an insulating dielectric material buildup on the target surface even in a very short period of time (a few seconds). Without the pulsed dc bias mechanism, this dielectric buildup would contaminate the target surface and significantly reduce the target material sputter yield, hence deposition rate would drop quickly. With the pulsed dc bias setup, any insulating dielectric material buildup on the target surface would be effectively discharged by the repulsive potential forces during the positive pulse period. In fact, even a small positive bias voltage $(\sim 20 \mathrm{~V})$, together with a relatively high frequency (1-75 $\mathrm{kHz}$ ) would be sufficient to discharge the buildup and stabilize the reactive sputtering process.

Another important function of the RBTIBD system is the heated sample stage, which is essential for effective material explorations. As shown in Figure 3.3, the sample stage 
is vertically positioned and rotates along the same axis shared by the ion source during the deposition. Tantalum was chosen as the material for the wafer carrier because of its relatively high melting temperature, low thermal expansion coefficient and non-ferrous nature [33]. The heating function is realized by an infrared heating lamp installed right behind the wafer carrier. The thermal output of the stage heating lamp is directly controlled by a variable voltage input, which is related and converted to a digital to analog count (DAC). The target surface temperature calibration is carried out by attaching a standard k-type thermocouple directly to the target surface and reading out the temperature value via a vacuum feedthrough. The infrared lamp is capable of heat up the stage up to $650^{\circ} \mathrm{C}$. The actual starting and ending of a deposition on the substrates is controlled by a mechanically driven stage shutter positioned $2 \mathrm{~mm}$ above the wafer carrier.

Overall, the compact design of the RBTIBD system reduces the overall complexity of the electronic and control system and makes it much easier in terms of the system maintenance compared to the highly automated BTIBD system. The new design of RBTIBD focuses more on the material exploration including complex oxides and metallic compounds. The equipped heating stage and pulsed dc bias scheme enable the rapid and effective combinatorial exploration of a wide range of phase space in various material systems, and several papers have already been published $[46,47]$ based on the initial study of some interesting complex oxides fabricated by the RBTIBD system. 


\subsection{Sample preparations}

Various wafer substrates were used in this dissertation work for the material and structural exploration of magnetic tunnel junctions (MTJs). For the deposition of full MTJ multilayer stacks, which was carried out mainly on the BTIBD system with automated recipe program, I used a thermally oxidized silicon wafer with an oxide layer

around $3000 \AA$, because the thermally oxidized silicon is relatively inexpensive and it is the preferred choice of mainstream industry. For the material exploration, like the $\tau$ phase MnAl alloy system as the potential ferromagnetic electrode of MTJs, it was carried out by the RBTIBD system, and various substrates were explored. The details of the work will be presented in chapter $\mathbf{8}$.

In any case, the substrate cleaning protocol as described in the following procedure was strictly followed for all the experiments. First step is the ultrasonic cleaning of the substrates with an acetone solution for 2 minutes. One clean aluminum dish, which is used to hold the substrates and acetone solution during the first step of cleaning, is rinsed by acetone to make sure the tiny aluminum particles on the surface of the dish will be washed away and won't be mixed in the acetone during the ultrasonic process to avoid contaminating the substrate surfaces. Ultrasonic processing longer than 2 minutes will damage the $\mathrm{SiO}_{2}$ surface as $\mathrm{I}$ observed under the microscope. After the ultrasonic cleaning with acetone, the substrates are picked out by a pair of clean nonmagnetic tweezers, rinsed in isopropanol to wash away the acetone residual and put into the second clean aluminum dish that is rinsed by isopropanol. The substrates are then immersed in isopropanol for the second 2 minutes ultrasonic cleaning process. After the two ultrasonic cleaning steps, the substrates are then picked out and blown dry by pure nitrogen gas and 
put into another clean aluminum dish for the final baking process. The substrates are baked at $120^{\circ} \mathrm{C}$ for 8 minutes on a hot plate to get rid of the surface moisture before putting into the vacuum chamber.

Two different substrate mounting methods are used depending on whether elevated temperature growth is utilized. For film growth in the BTIBD system, where the sample stage is water cooled, the substrates are usually taped on the corner to the wafer holder. While in the case of the heating stage in the RBTIBD system, a silver paint is used to fix the substrates to the Ta wafer carrier. The wafer carrier with substrates is then baked at $120^{\circ} \mathrm{C}$ for 8 minutes to get rid of the surface moisture and cure the silver paint as well.

The BTIBD system was originally designed as a production tool with a highly automated recipe control system, suitable for the automatic deposition of multilayer structures. The deposition parameters including the ion source current and voltage, anode and cathode gas flow rates, target bias voltage and current, etc. are all programmed into a single subroutine recipe. Multiple subroutines are then combined in sequence to form the master recipe to precisely control every step of the multilayer deposition. Before starting the actual run of the automatic recipe, the ion source needs to be turned on first. This is done by first purging $10 \mathrm{sccm}$ ultra high purity (UHP) Ar gas through the cathode for 30 minutes, followed by the degas procedure for another 30 minutes, in which the tungsten filament inside the cathode is heated by the passing current to outgas any possible contaminations on its surface. After the one hour purging and degas preparation, the ion source is ignited by introducing UHP Ar gas to both cathode and anode while applying certain voltages on them both as well. Up to twelve pieces of wafer carriers are loaded into a cassette in the load lock chamber to start the film depositions. After pumping the 
cassette chamber down to high vacuum that is compatible to the vacuum level in the transport chamber, the isolation valve between the two chambers opens up. After that, the robot arm reaches into the cassette chamber to pick up the designated piece of wafer carrier from the one of twelve slots as programmed, and then transfer the same wafer carrier into the processing chamber for the actual deposition as programmed in the master recipe.

Compared with the highly automated BTIBD system where a programmed recipe is used to precisely control the thin film and multilayer structure growth in the atomic level, the compact design of the RBTIBD system allow more freedom in terms of manual control, and the typical operation procedure will be presented in detail in section 8.2.

The post-deposition annealing treatment is essential for making good quality MTJs [23] as well as forming correct $\tau$ phase of MnAl thin films [48]. In order to anneal our samples with an external magnetic field capability, we built two annealing systems of our own, one using forming gas (mixture of 95\% $\mathrm{Ar}$ and $5 \% \mathrm{H}_{2}$ ) and the other vacuum. The forming gas furnace is equipped with magnetic field up to $5 \mathrm{kOe}$, and the forming gas with 5\% hydrogen protects the sample surface from oxidation effectively The vacuum furnace can provide annealing up to $500^{\circ} \mathrm{C}$ with very precious digital control of the temperature. The vacuum base pressure is in the low $10^{-6}$ Torr range and a magnetic field up to $400 \mathrm{Oe}$ is provided by two permanent ferromagnets. In terms of temperature control, our own vacuum furnace has the ramp up rate of $5^{\circ} \mathrm{C} / \mathrm{min}$ with precious digital control, and slow natural cooling down rate less than $2^{\circ} \mathrm{C} / \mathrm{min}$. The furnace with forming gas has much higher temperature ramp up rate around $25^{\circ} \mathrm{C} / \mathrm{min}$, and similar cooling down rate of $25^{\circ} \mathrm{C} / \mathrm{min}$ when a cooling fan is applied during the cooling process. Another 
method of heat treatment is the rapid thermal annealing (RTA), in which the temperature ramp up and cooling rate is much faster compared with our own furnaces. The RTA treatment was carried out by using a commercial RTA system, where both forming gas and vacuum (base pressure $\sim 10^{-6} \mathrm{~T}$ ) modes can be used. The RTA temperature ramp up rate can easily reach $500^{\circ} \mathrm{C} / \mathrm{min}$, and the temperature is maintained very stably with the digital power control system during the annealing process. The step of cooling down from high temperature like $500^{\circ} \mathrm{C}$ also takes only a few minutes with the RTA method. 


\section{Exchange bias for magnetic tunnel junctions}

\subsection{Introduction of exchange bias}

When a thin film bilayer system with a ferromagnetic (FM) and an antiferromagnetic (AFM) layer interface is cooled through the Néel temperature $\left(T_{N}\right)$ of the AFM (with the Curie temperature $T_{C}$ of the FM larger than $T_{N}$ ) in a magnetic field, a new type of anisotropy called "exchange bias" (EB) is induced at the interface between the FM and AFM layers [26]. This EB phenomenon was first discovered by Meiklejohn and Bean more than 50 years ago when studying the Co particles embedded in their native AFM oxide $(\mathrm{CoO})[49,50]$. The magnetization loop $\mathrm{M}(\mathrm{H})$ of the FM layer in the FM/AFM bilayer structure could be shifted from zero due to the EB anisotropy. This unique feature of EB makes the FM/AFM bilayer system a key component in spintronic devices [18] such as spin-valves and magnetic tunnel junctions (MTJs). Mainly, the FM/AFM EB bilayer is used as the reference layer with fixed magnetization to achieve the parallel/antiparallel configuration of the magnetization when switching the free layer of the spin valves or MTJs. Figure 4.1(a) shows a representative MTJ stack deposited by the BTIBD system with NiCoFe/FeMn EB bilayer as the fixed layer. Figure 4.1(b) shows the separate switching of the free and fixed layer, with the fixed layer loop center shifting $\sim 30$ Oe off the origin due to the EB anisotropy. 


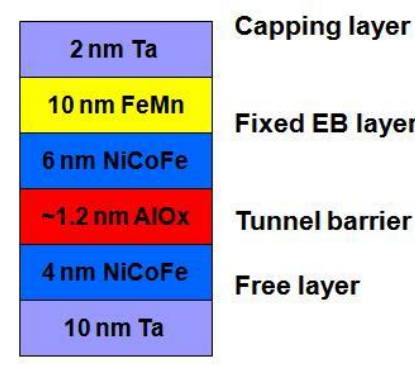

(a)

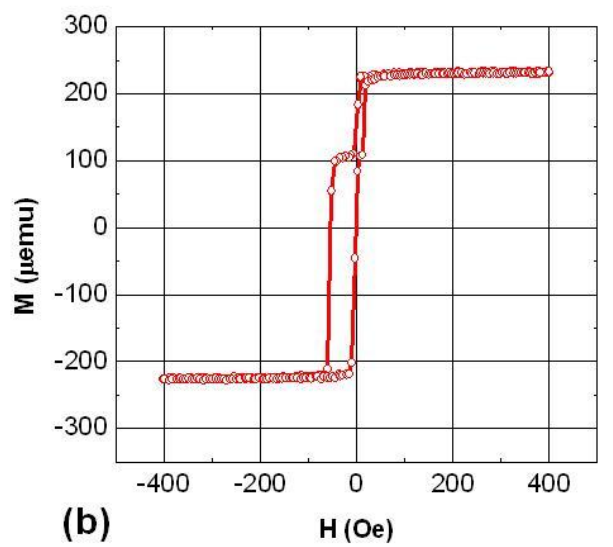

(b)

$\mathrm{H}(\mathrm{Oe})$

Figure 4.1 (a) Representative MTJ stack with exchange biased fixed layer; (b) M(H) hysteresis loop of the MTJ stack showing the separate switching of the free layer and exchange biased fixed layer

The mechanism of the EB anisotropy could be understood qualitatively by assuming an exchange interaction at the FM/AFM interface as illustrated in Figure 4.2 [26]. When a magnetic field is applied along the direction of the FM/AFM interface at the temperature $T_{N}<T<T_{C}$, the spins in the FM layer align with the field while the AFM spins remain random (Figure 4.2(i)) since the temperature is above the Néel temperature $\mathrm{T}_{\mathrm{N}}$ of the AFM material. When the bilayer system is cooled through $\mathrm{T}_{\mathrm{N}}$ with the presence of the external magnetic field, the AFM spins at the FM/AFM interface tend to align parallel to the spins inside the FM layer, assuming the ferromagnetic coupling between spins from AFM and spins from FM along the surface. Starting from the second layers, the spins inside the AFM material follow the AFM order so as to produce zero net magnetization as shown in Figure 4.2(ii). When the external filed becomes negative, firstly the spins in the FM layer start to rotate according to the external field. However, due to the induced EB anisotropy at the FM/AFM interface, the interfacial spins on the 
AFM side, which is usually very stable due to much larger AFM coupling within the AFM material, tend to align the interfacial spins on the FM side and keep them from rotating with the negative external field. In other words, the AFM spins at the interface exert a microscopic torque on the FM spins, to keep them in their original position (ferromagnetically aligned at the interface) as shown in Figure 4.2(iii) [26]. The microscopic torque due to the EB anisotropy keeps the FM spins in a single stable state, so the EB anisotropy is also called unidirectional exchange anisotropy, and the direction of the EB is determined by the external field direction in the initial cooling step. Therefore, compared with a single FM layer, the field required to switch a FM, sharing an interface with an AFM layer will be larger, since extra field is needed to overcome the microscopic torque due to the EB anisotropy.

With a larger field that is sufficient to overcome the EB anisotropy, the FM spins in the FM/AFM structure will be finally switched to the negative direction to align with the field, as shown in Figure 4.2(iv). This state, with the interfacial spins on the FM side antiferromagnetically coupled to the spins on the AFM side, is a high energy unstable state. Therefore, once the external field is switched back to the original positive direction, a much smaller field is needed to start the switching process of the spins in the FM layer, because the microscopic torque exerted on the FM spins is now in the same direction of the external field due to the antiferromagnetic coupling of the interfacial spins from both sides. The earlier switching due to the same unidirectional exchange coupling is illustrated in Figure 4.2(v). Overall, the FM/AFM bilayer system with EB anisotropy acts like if there is an extra (internal) biasing field [26], which effectively shift the hysteresis loop of the FM layer in the field axis as shown in Figure 4.2. 


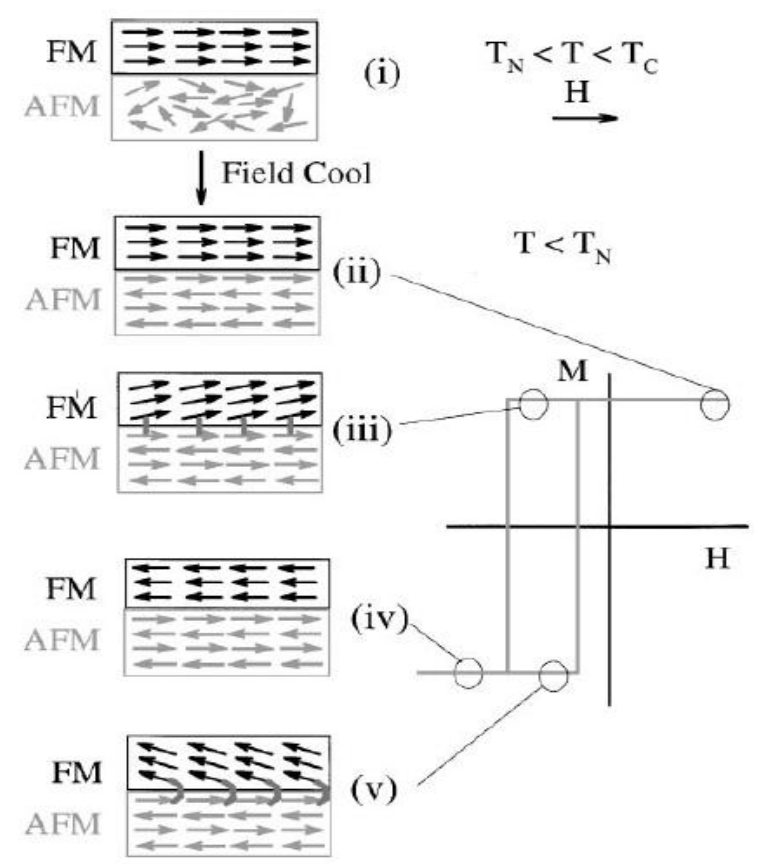

Figure 4.2 Schematic diagram of the spin configuration of an FM/AFM bilayer at different stage (i)(v) of an exchange biased hysteresis loop. Note that the spin configurations are just a simple cartoon to illustrate the effect of the coupling and they are not necessarily accurate portraits of the actual rotation of the FM or AFM magnetizations. (Adapted from Ref. [26])

\subsection{Exchange bias in $\mathrm{CoFe} / \mathrm{FeMn} / \mathrm{CoFe}$ trilayers}

\subsubsection{Introduction}

It is generally recognized that the EB phenomenon is an interfacial effect, in that the spins in the AFM material are regarded as static and rigid throughout the magnetic reversal process of the FM layer, as proposed by the simple theoretical models [49, 50]. In such models, the exchange interaction between the FM spins and the interfacial spins from the AFM gives rise to an effective static magnetic field $\mathrm{H}_{\mathrm{EB}}$, also called the EB field that shifts the hysteresis loop on the FM layer, in apparent agreement with the 
experimental results [51]. However, these simple models didn't explain the experimental results very well. For example, the simple models predicted enormous values of the EB field $\mathrm{H}_{\mathrm{EB}}$ that is several orders of magnitude larger than the observed values. Also, recent experiments studying the motion of the a single domain wall in a FM/AFM bilayer with a wedged FM layer showed an acute asymmetry between the magnetization reversal from positive to negative field and that from negative to positive field $[52,53]$. This asymmetric magnetization reversal was not expected in the simple theoretical models with static AFM spin structures. Furthermore, the EB field $\mathrm{H}_{\mathrm{EB}}$ was found to be strongly dependent on the thickness of the AFM layer even for very large thickness [54]. Also, the EB was found to exist even when a nonmagnetic spacer such as $\mathrm{Ag}, \mathrm{Au}$ or $\mathrm{Cu}$ was inserted in between the AFM and FM layer [55]. All of these experiments showed that the AFM bulk might also play a very important role in the EB phenomenon besides the interfacial AFM spins. So the static AFM spin model was not necessary correct and further investigations are needed to explore the underlying physics of this EB effect.

One good structure for the further exploration of underlying physics of the EB effect is the FM/AFM/FM trilayer system which has two FM/AFM interfaces that couple with each other. In FM/AFM/FM trilayers, a bulk characteristic of EB would imply that the $\mathrm{EB}$ at the FM/AFM interface and that from the AFM/FM interface are correlated and propagate from one to the other. However, recent studies on such trilayers gave quite different results depending on the material specifics and magnetic heat treatments. Using a field cooling (FC) procedure at the plateau field that separates the minor switching loops of the two FM layers in Py/FeMn/Co trilayers, Yang and Chien [51] observed that the top and bottom EB systems were coupled via a spiraling spin structure across the 
intervening FeMn layer. Leung and Blamire [56] later suggested that such a microscopic AFM spin spiral was due the specific FC treatment applied in the work, but not a universal feature of the EB trilayers. While no sign of bias propagation was observed in $\mathrm{NiFe} / \mathrm{FeMn} / \mathrm{Co}$ trilayers grown in a low field $(\sim 5 \mathrm{Oe})$ [57], it was seen in those that were field cooled in $1 \mathrm{kOe}$ from above the blocking temperature [56]. It was reported that no propagation of spin order was observed between the interfaces in $\mathrm{Co} / \mathrm{FeMn} / \mathrm{CuNi}$ structures grown in an external field of about 200 Oe.

Our work in a FM/AFM bilayer structure shows that there is a large difference in EB coupling magnitude between $\mathrm{FeMn} / \mathrm{CoFe}$ and $\mathrm{CoFe} / \mathrm{FeMn}$ structures due to the influence of the magnetized $\mathrm{CoFe}$ underlayer on the establishment of the AFM ordering of FeMn. Remarkably, it was observed that adding a seed $\mathrm{CoFe}$ thin layer to the $\mathrm{FeMn} / \mathrm{CoFe}$ bilayer significantly improved the bias field $\mathrm{H}_{\mathrm{EB}}$ of the top CoFe layer. On the other hand, deposition of a top CoFe on the CoFe /FeMn bilayer structure enhanced the $\mathrm{H}_{\mathrm{EB}}$ of the bottom CoFe layer as well. The top and bottom CoFe layers in the trilayers both showed an enhanced bias field $\mathrm{H}_{\mathrm{EB}}$ with decreasing thickness of the shared FeMn layer. These results support the presence of significant propagation of EB in the FeMn layer in the $\mathrm{CoFe} / \mathrm{FeMn} / \mathrm{CoFe}$ trilayers. Here the study of $\mathrm{CoFe} / \mathrm{FeMn} / \mathrm{CoFe}$ trilayers not only furthered our understanding of the EB mechanism, but also provided a robust trilayer system with enhanced EB effect that are used as the fixed reference layers in the MTJ stack fabrication and the investigation of MTJ properties. 


\subsubsection{Experiments}

The CoFe/FeMn, FeMn/CoFe bilayer structures and the symmetric $\mathrm{CoFe} / \mathrm{FeMn} / \mathrm{CoFe}$ trilayers were grown at room temperature (RT) using the BTIBD system introduced in section 3.1. An in situ magnetic field $~ 50$ Oe was applied along the substrate surface during the deposition to create the easy axis of the FM layers. The CoFe and FeMn target compositions are $\mathrm{Co}_{95} \mathrm{Fe}_{5}$ and $\mathrm{Fe}_{50} \mathrm{Mn}_{50}$, respectively. All of the samples were grown on Si wafers. Before the actual EB stack deposition, a seed layer of Ta (5 $\mathrm{nm}$ ) (for $\mathrm{CoFe} / \mathrm{FeMn}$ bilayer structures and trilayers) or $\mathrm{Cu}(5 \mathrm{~nm}$ ) (for $\mathrm{FeMn} / \mathrm{CoFe}$ bilayers) was deposited. After the deposition of the EB stacks, all samples were capped with a Ta $(5 \mathrm{~nm})$ layer except for the $\mathrm{CoFe} / \mathrm{FeMn}$ bilayers that used a cap of $5 \mathrm{~nm} \mathrm{Cu}$. The reason to use $\mathrm{Cu}$ capping layers was to promote the $\gamma$-fcc FeMn phase which is required for the formation of $\mathrm{EB}$. The samples subject to FC were heated from RT to 230 ${ }^{\circ} \mathrm{C}$ in $4.5 \mathrm{~min}$ and then cooled to RT in a magnetic field of $3 \mathrm{kOe}$ using our forming gas $\left(95 \% \mathrm{~N}_{2}+5 \% \mathrm{H}_{2}\right)$ furnace. The EB field $\mathrm{H}_{\mathrm{EB}}$, defined as $\mathrm{H}_{\mathrm{EB}}=\left|\mathrm{H}_{\mathrm{c} 1}+\mathrm{H}_{\mathrm{c} 2}\right| / 2$, was measured at $305 \mathrm{~K}$ for all the samples in both as-deposited and field-cooled states. Here, $\mathrm{H}_{\mathrm{c} 1}$ and $\mathrm{H}_{\mathrm{c} 2}$ are the coercivity values determined on the opposite field sweep directions that were measured at $\mathrm{M} / \mathrm{M}_{\mathrm{S}}=0$ for the bilayer structures and at $\mathrm{M} / \mathrm{M}_{\mathrm{S}}= \pm 0.5$ separately for the two CoFe layers in the trilayers.

\subsubsection{Results and discussions}

Typical $\mathrm{M}(\mathrm{H})$ loops of our bilayer structure and trilayers are presented in Figure 4.3 for $\mathrm{FeMn}(10) / \mathrm{CoFe}(4), \mathrm{CoFe}(4) / \mathrm{FeMn}(10)$, and $\mathrm{CoFe}(4) / \mathrm{FeMn}(10) / \mathrm{CoFe}(4)$ samples. 
First of all, as for as-deposited samples, it has been commonly observed that the EB is much stronger in the $\mathrm{CoFe} / \mathrm{FeMn}$ bilayers than in FeMn/CoFe structures. This result is clearly demonstrated in Figure 4.3(a) and Figure 4.3(b) where a large difference in $\mathrm{H}_{\mathrm{EB}}$ between $\mathrm{FeMn}(10) / \mathrm{CoFe}(4)\left(\mathrm{H}_{\mathrm{EB}}=44 \mathrm{Oe}\right)$ and $\mathrm{CoFe}(4) / \mathrm{FeMn}(10)\left(\mathrm{H}_{\mathrm{EB}}=190 \mathrm{Oe}\right)$ bilayers is observed. This effect seems to be caused by the changes in the micromagnetic structure due to the magnetic interactions rather than in the crystalline structure and texture of the FeMn layer, which is associated with the underlayer effect, since a $\mathrm{Cu}$ seeding layer was used to promote the $\gamma$-fcc FeMn phase. In the case of the CoFe/FeMn bilayer, since the CoFe layer was first deposited with an applied in situ field $\sim 50$ Oe, the CoFe film was already fully magnetized in its easy axis direction. Thus the strong polarizing field produced by the CoFe surface magnetization tends to compete against the formation of the AFM ordering. In this case, the uncompensated spins of the AFM FeMn layer at the interface were easily created when FeMn was grown on the saturated CoFe in a magnetic field. On the other hand, depositing CoFe on an already stable FeMn layer may have a minimal effect on its well established AFM ordering. In this case, a significantly weaker EB was created. A much higher in situ field might be required to create as strong an $\mathrm{EB}$ in $\mathrm{FeMn} / \mathrm{CoFe}$ bilayers as that in the $\mathrm{CoFe} / \mathrm{FeMn}$ structure in order to overcome the AFM ordering of the FeMn layer. Within this context, the concentration of uncompensated spins may be one factor that determines the exchange coupling between the AFM and FM layers across their interface. 


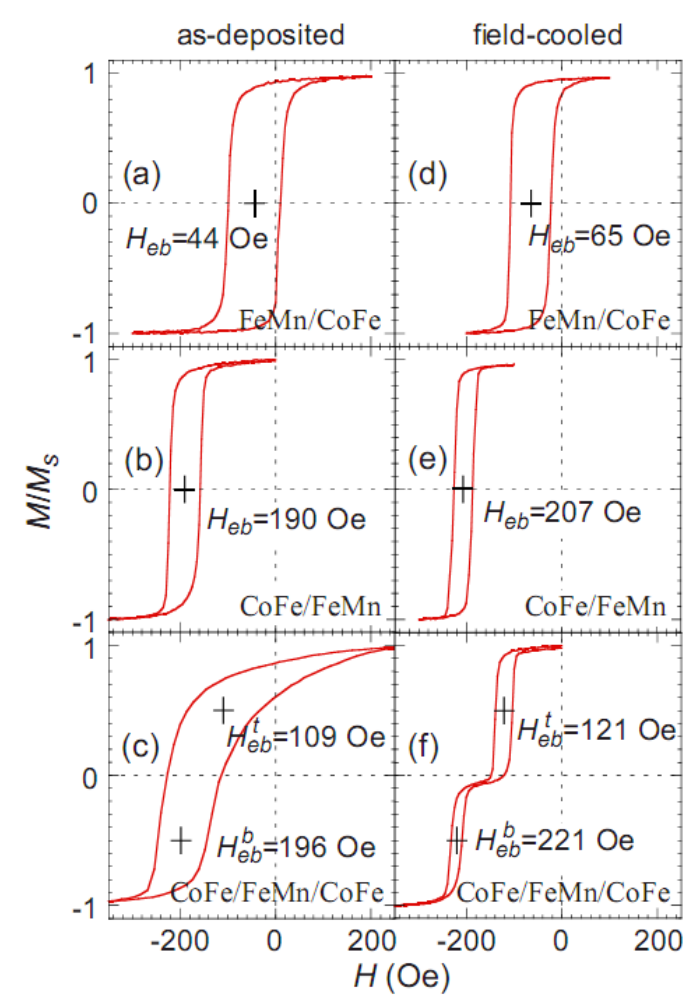

Figure 4.3 $\mathrm{M}(\mathrm{H})$ loops of the bilayer structure $\mathrm{Si} / \mathrm{Cu}(5) / \mathrm{FeMn}(10) / \mathrm{CoFe}(4) / \mathrm{Ta}(5)$ (a) and (d); $\mathrm{Si} / \mathrm{Ta}(5) / \mathrm{CoFe}(4) / \mathrm{FeMn}(10) / \mathrm{Cu}(5)(b)$ and (e); $\mathrm{Si} / \mathrm{Ta}(5) / \mathrm{CoFe}(4) / \mathrm{FeMn}(10) / \mathrm{CoFe}(4) / \mathrm{Ta}(5)$ trilayers (c) and (f). Samples (a) (b) and (c) were as-deposited, and samples (d) (e) (f) were field cooled. The crosses marked the center of the main or minor loops where $\mathbf{H}_{\mathrm{EB}}$ was determined.

For the trilayers, by varying the relative thickness of the two CoFe layers, the minor loops for the top and bottom CoFe layers could be easily identified. The hysteresis loop of the as-deposited $\mathrm{CoFe}(4) / \mathrm{FeMn}(10) / \mathrm{CoFe}(4)$ trilayers in Figure 4.3(c) shows only a slight increase in the EB field $H_{E B}^{b}$ of the bottom $\mathrm{CoFe}(4)$ layer (lower minor loop; $H_{E B}^{b}=$ 196 Oe), compared to the corresponding $\mathrm{CoFe} / \mathrm{FeMn}$ bilayer structure $\left(\mathrm{H}_{\mathrm{EB}}=190 \mathrm{Oe}\right)$ shown in Figure 4.3(b). In contrast, a huge increase of EB field is induced in the top CoFe layer. The broad magnetization reversals of the top CoFe layer (up minor loop) indicates that its exchange coupling with the FeMn layer is not uniform and partially 
randomized. A major part of the layer seems to switch its magnetic moments synchronously with the bottom CoFe layer. The nominal EB field of the top part $H_{E B}^{t}$ measured at $\mathrm{M} / \mathrm{M}_{\mathrm{S}}=0.5$ is $109 \mathrm{Oe}$, which is much higher than the value of $44 \mathrm{Oe}$ obtained for the corresponding $\mathrm{FeMn} / \mathrm{CoFe}$ bilayer structure shown in Figure 4.3(a). These results unambiguously indicate that the EB effect is strongly improved in the top CoFe layer in the presence of the bottom $\mathrm{CoFe}$ layer. It is unlikely that the bottom $\mathrm{CoFe}$ layer would cause a large improvement of the $\gamma$-fcc AFM phase in the FeMn layer than by a $\mathrm{Cu}$ underlayer. On the other hand, the presence of the top $\mathrm{CoFe}$ is not expected to make any influence on the crystalline structure of the FeMn layer underneath. Therefore, the improvements of the EB effect of both CoFe layers must be indicative of a magnetic coupling between the two $\mathrm{CoFe} / \mathrm{FeMn}$ and $\mathrm{FeMn} / \mathrm{CoFe}$ systems sharing the same intervening FeMn layer. 


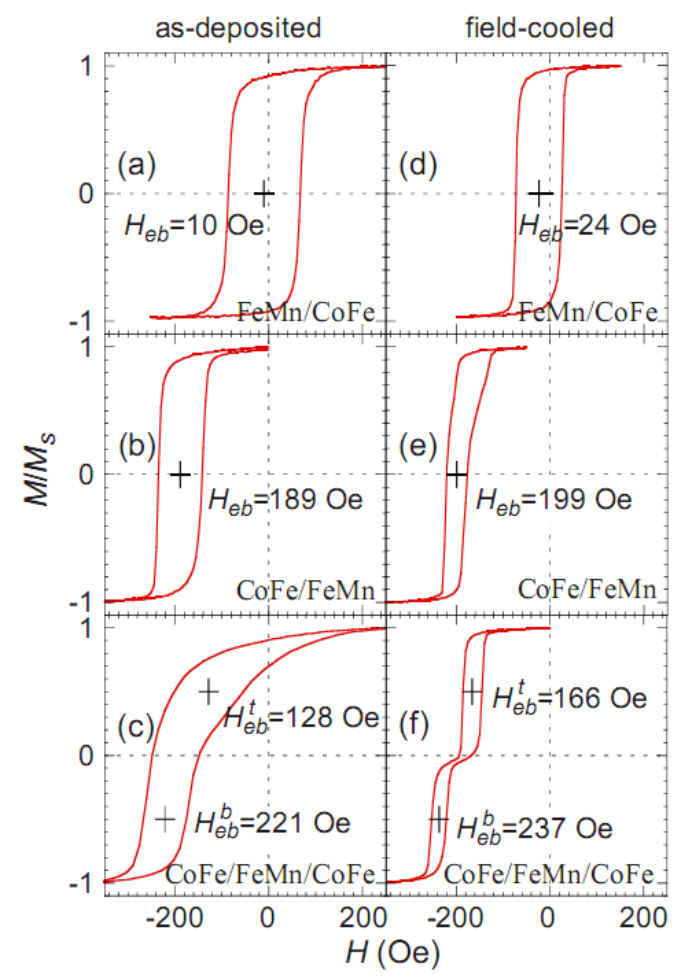

Figure 4.4 $\mathrm{M(H)}$ loops of the bilayer structure $\mathrm{Si} / \mathrm{Cu}(5) / \mathrm{FeMn}(6) / \mathrm{CoFe}(4) / \mathrm{Ta}(5) \quad$ (a) and (d); $\mathrm{Si} / \mathrm{Ta}(5) / \mathrm{CoFe}(4) / \mathrm{FeMn}(6) / \mathrm{Cu}(5)$ (b) and (e); $\mathrm{Si} / \mathrm{Ta}(5) / \mathrm{CoFe}(4) / \mathrm{FeMn}(6) / \mathrm{CoFe}(4) / \mathrm{Ta}(5)$ trilayers (c) and (f). Samples (a) (b) and (c) were as-deposited, and samples (d) (e) (f) were field cooled. The crosses marked the center of the main or minor loops where $H_{\mathrm{EB}}$ was determined.

Nevertheless, the increase of only 6 Oe (or 3\%) in the EB field of the bottom CoFe layer from bilayer structure to trilayers shown in Figure $4.3(\mathrm{~b}) \&$ (c) is somewhat too small to be conclusive. Therefore, another set of samples were deposited to further investigate the mechanism of EB propagation. The second set of samples with thinner FeMn middle layers were fabricated based on the fact that if the top and bottom EB systems are magnetically coupled, the EB field $H_{E B}^{t}$ and $H_{E B}^{b}$ would increase with decreasing the FeMn layer thickness $\mathrm{t}_{\mathrm{FeMn}}$. Figure 4.4 plots the hysteresis loops of similar bilayer structures and trilayers but with a thinner $(6 \mathrm{~nm})$ FeMn middle layer. As expected, 
when decreasing $\mathrm{t}_{\mathrm{FeMn}}$ from 10 to $6 \mathrm{~nm}, H_{E B}^{t}$ increased from 109 to 128 Oe and $H_{E B}^{b}$ from 196 Oe to 221 Oe when comparing Figure 4.3(c) and Figure 4.4(c). Moreover, $H_{E B}^{b}$ of the $\mathrm{t}_{\mathrm{FeMn}}=6 \mathrm{~nm}$ trilayers is now $32 \mathrm{Oe}$ (or 17\%) higher than that of the corresponding CoFe layer in the bilayer structure. It is worth noting here that while dipolar (or "orangepeel" type) coupling between the two CoFe layers may not be avoidable in our trilayers, it cannot be responsible for the increase of $H_{E B}^{b}$ with decreasing $\mathrm{t}_{\mathrm{FeMn}}$. Our data (not shown here) indicate that due to the dipolar coupling, $H_{E B}^{b}$ starts to decrease when $\mathrm{t}_{\mathrm{FeMn}}$ is decreased to below $6 \mathrm{~nm}$ (e.g., $H_{E B}^{b}=204$ and 173 Oe for $\mathrm{t}_{\mathrm{FeMn}}=5$ and $4 \mathrm{~nm}$, respectively). A huge increase in the EB is also obtained for the top CoFe layer in the trilayers shown in Figure 4.4(c) with reference to the bilayer structure in Figure 4.4(a). It is very interesting that as $t_{\mathrm{FeMn}}$ decreases, while the $\mathrm{EB}$ fields are reduced in both top and bottom CoFe bilayer structures, they are strongly increased in the corresponding trilayers. All of these facts convincingly suggest that there exists a mutual propagation of EB between the top and bottom interfaces through the intermediate FeMn layer. Even for $\mathrm{t}_{\mathrm{FeMn}}$ of up to $25 \mathrm{~nm}$, the top CoFe layer EB is still induced by the bottom one. Although the shape of the $\mathrm{M}(\mathrm{H})$ loops of the trilayers in Figure 4.3(c) and Figure 4.4(c) look rather similar to those observed by yang and Chien [51], our angular measurements indicate that both the CoFe layers have the same easy axis as that initially created by the external field applied during the deposition, thus avoiding any possibility of a spiraling magnetic structure in these samples.

As shown in Figure 4.3(d) - (f) and Figure 4.4(d) - (f), it is surprising that field cooling the samples from $230^{\circ} \mathrm{C}$ while applying an external field of $3 \mathrm{kOe}$ only slightly improves their EB fields. This indicates that the EB states established in the as-deposited 
samples were already close to equilibrium. The biggest change is observed for the top CoFe layers in both the FeMn(10 nm) and FeMn(6 nm) trilayers as shown in Figure 4.3(f) and Figure 4.4(f), respectively. In both cases, the EB becomes uniform and greatly improved, due to a realignment of the randomized uncompensated spins by the FC process. Qualitatively, the behaviors of the field-cooled samples are in general the same as that of the as-deposited ones. The $\mathrm{FC}$ temperature was also varied from 180 to $300{ }^{\circ} \mathrm{C}$ and no significant changes in terms of the EB hysteresis loops were observed. The large difference in the EB between the field-cooled $\mathrm{CoFe} / \mathrm{FeMn}$ and $\mathrm{FeMn} / \mathrm{CoFe}$ bilayer structures (and between the top and bottom interfaces in the trilayers as well) is a striking feature that would imply that the FC may have resulted in the coupling of CoFe layers and uncompensated spins without significantly increasing their concentration. It is therefore possible that $3 \mathrm{kOe}$ cooling field is still too low to bring about equal EB fields for the two EB systems whether in the bilayer structure or trilayers.

Regarding the shape of the $\mathrm{M}(\mathrm{H})$ curves of the as-deposited trilayers as shown in Figure 4.3(c) and 4.4(c), one would have concern that the apparent increase in $H_{E B}^{b}$ is probably an artifact of the large coercivity and broad loop of the top CoFe layer. However, a numerical deconvolution of the trilayers $\mathrm{M}(\mathrm{H})$ curves, using the loop of the $\mathrm{CoFe} / \mathrm{FeMn}$ bilayer structures as shown in Figure 4.3(b) and 4.4(b) as that of the bottom CoFe layers, give us unreasonably distorted loop for the top CoFe layer though less distortion is obtained when $\mathrm{H}_{\mathrm{EB}}$ of the $\mathrm{CoFe} / \mathrm{FeMn}$ bilayer structure is shifted toward $H_{E B}^{b}$. This would imply that the increase in $H_{E B}^{b}$ is an intrinsic change of the bottom CoFe layer in the trilayer structure, as is also supported by the results of field cooling as shown 
in Figure 4.3(f) and 4.4(f), where a substantial increase in $H_{E B}^{b}$ is observed while the top CoFe layer loop is sharp, narrow, and has $H_{E B}^{t}$ significantly below $H_{E B}^{b}$.

\subsubsection{Summary}

First of all, the study of the EB propagation furthered our understanding of the underlying physics involved in the EB phenomenon. Earlier studies relying on simple theoretical models largely regarded the EB as an interfacial phenomenon. However, in our study, it is found that although the uncompensated spins are created near the FM/AFM interface, they also spread over the AFM layer to the top AFM/FM interface, where the creation of uncompensated spins is less favored, leading to a strong improvement of the EB of the top FM layer. On the other hand, the top FM layer may also contribute a certain amount of uncompensated spins, resulting in an increase of the bottom FM layer EB. Our results here underline the importance of the concentration, as well as the distribution, of the uncompensated spins in the EB systems and demonstrated that there exists a propagation of EB within the AFM middle layer in the FM/AFM/FM trilayer structures.

Also, the investigation of $\mathrm{EB}$ in the bilayer structure and trilayers involving $\mathrm{CoFe}$ and FeMn has shown that the uncompensated spins are created favorably when an AFM is deposited on a magnetized FM layer. This finding is of valuable practical importance and guided us to the creation of FM/AFM/FM trilayers, which becomes a key component of our MTJ stacks deposited by the BTIBD system as the reference layer with fixed magnetization. 


\section{Oxidation of the ferromagnetic bottom electrode in magnetic tunnel junctions}

\subsection{Introduction}

Since the discovery of room temperature (RT) large tunnel magnetoresistance (TMR) in magnetic tunnel junctions (MTJs) [1, 2], intensive research has been carried out on this subject. This is due in large part to the various potential technological applications utilizing the high TMR performance [18], which is quickly approaching the theoretically predicted value of over $1000 \%$ in Fe/MgO/Fe MTJ structure [20]. Recently, a TMR value of $605 \%$ was reported in a pseudo spin valve annealed at high temperature [58], and a record high TMR value of $1056 \%$ was reported in a double barrier MTJ structure with $\mathrm{MgO}$ as the barrier material [59]. However, the performance of MTJs is well known to be very sensitive to fabrication conditions, and significant challenges remain for fabrication of reliable high quality MTJs.

Among these challenges, one critical step is the formation of oxide tunnel barrier. The barrier oxidation step needs precise control due to the thickness of the barrier, which is usually less than $2 \mathrm{~nm}$. If the barrier is under-oxidized, the un-oxidized metal could significantly diminish the tunneling spin polarization, even if only a few monolayers thick. On the other hand, if the barrier is over oxidized, oxidation of the ferromagnetic electrode could occur. This is also detrimental to the polarized spin tunneling efficiency through the electrode/barrier interface [27]. In the current stage of development, most ferromagnetic electrodes used in the MTJ structures are Co rich alloys like CoFe and CoFeB. In the case of barrier over-oxidation, the bottom electrode surface will oxidize 
and antiferromagnetic $\mathrm{CoO}$ will be formed at the bottom electrode/barrier interface. When such a system is cooled down in a magnetic field from RT through the Néel temperature of $\mathrm{CoO}(293 \mathrm{~K})$, a typical CoO/FM EB system will be formed $[49,50]$. In this study, the EB system is utilized as an indicator of barrier over-oxidation. Specifically, four parameters associated with the observation of EB as the detection parameters of barrier over-oxidation will be identified, including the coercivity, EB field, training effect and uncompensated spin density. The detection sensitivity of these four detection parameters will be investigated and discussed in detail, and the one with highest sensitivity will be picked for practical use. Also, the influence of annealing has on the barrier oxidation will be investigated using the detection parameters. Conclusions on oxygen migration during annealing can be drawn from this magnetic study. This conclusion is consistent with the results of previous reports using advanced spectroscopic methods [60]. It is proposed that the magnetic measurement developed in this study can serve as an easy and effective way of detecting the tunnel barrier oxidation quality during the MTJ fabrication process. In particular, it will be a useful tool for indentifying the process windows when exploring new oxide barrier materials other than the well established $\mathrm{Al}_{2} \mathrm{O}_{3}$ and $\mathrm{MgO}$.

\subsection{Experiment}

The partial MTJ stacks used in this study were deposited using our biased target ion beam deposition (BTIBD) system. The base pressure of the system was $2 \times 10^{-7}$ Torr, and the processing pressure was $\sim 7 \times 10^{-4}$ Torr with an $80 \mathrm{Sccm}$ Ar flow during deposition. A 
magnetic field of 50 Oe was applied parallel to the plane of the film in situ during the film growth. This preferentially sets the magnetic easy axis of the ferromagnetic (FM) layers. The stacks have the following structure as shown in the inset of Figure 5.1(b): substrate/Ta(6 nm)/FM(5 nm)/Mg(2.4 nm, with post-oxidation $) / \mathrm{Ta}(6 \mathrm{~nm})$. The substrate was thermally oxidized silicon, and $\mathrm{Co}_{95} \mathrm{Fe}_{5}$ or $\mathrm{Co}_{60} \mathrm{Fe}_{20} \mathrm{~B}_{20}$ was used as the FM bottom electrode materials. The $\mathrm{MgO}$ barrier was formed by $\mathrm{Ar} / \mathrm{O}_{2}$ plasma oxidation after deposition of a thin $\mathrm{Mg}$ metallic layer. For each of the two FM electrode materials, samples with different oxidation times were fabricated to study the sensitivity of various detection parameters. To investigate the influence of annealing on the magnetic properties, two different temperatures $\left(200^{\circ} \mathrm{C}\right.$ and $\left.350^{\circ} \mathrm{C}\right)$ with an external field of $3 \mathrm{kOe}$ were applied. The direction of the external field was parallel to the plane of the films along the same direction as was during the film deposition. The samples were protected from oxidation during annealing by a flowing forming gas $\left(95 \% \mathrm{~N}_{2}+5 \% \mathrm{H}_{2}\right)$. Magnetic properties were measured using a Quantum Design PPMS-6000 system at both room temperature $(\mathrm{RT})$ and $11 \mathrm{~K}$. For the low temperature measurement, all samples were field-cooled from RT with an applied magnetic field of $3 \mathrm{kOe}$ along their easy axes. The hysteresis loops of each sample were repeated three times at $11 \mathrm{~K}$ in order to study the training effect associated with the exchange bias (EB) effect. 


\subsection{Results and discussion}

\subsubsection{Parameters related to EB effect}

Figure 5.1 shows the magnetic hysteresis loops of two representative samples with bottom FM electrodes of $\mathrm{CoFe}$ (Figure 5.1(a)) and $\mathrm{CoFeB}$ (Figure 5.1(b)), respectively. Both samples were oxidized with $\mathrm{Ar} / \mathrm{O}_{2}$ plasma for 90 seconds after the $\mathrm{Mg}$ metal layer deposition in order to form the $\mathrm{MgO}$ barrier. Also shown in Figure 5.1 are the loops from the corresponding reference sample with no oxidation step was carried out for the $\mathrm{Mg}$ metal layers. In Fig. 1, the loops in red were measured at RT and they represent each of the two oxidized sample with $\mathrm{CoFe}$ and $\mathrm{CoFeB}$ bottom electrodes, respectively. The remaining three loops in the colors of green, blue and magenta are the three repeated loops of each sample after cooling down to $11 \mathrm{~K}$ with a $3 \mathrm{kOe}$ field along the film easy axis. Similar $11 \mathrm{~K}$ measurement with three cycling loops was carried out for the corresponding reference sample as well. These loops were marked in black color and they all overlap with each other. 


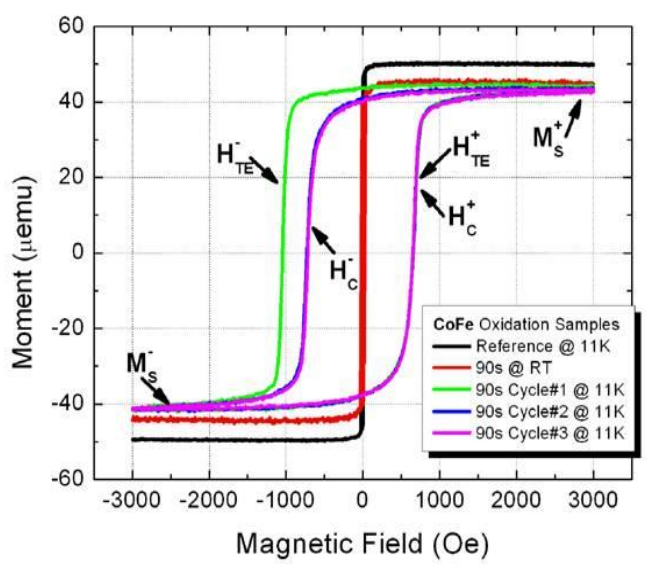

(a)

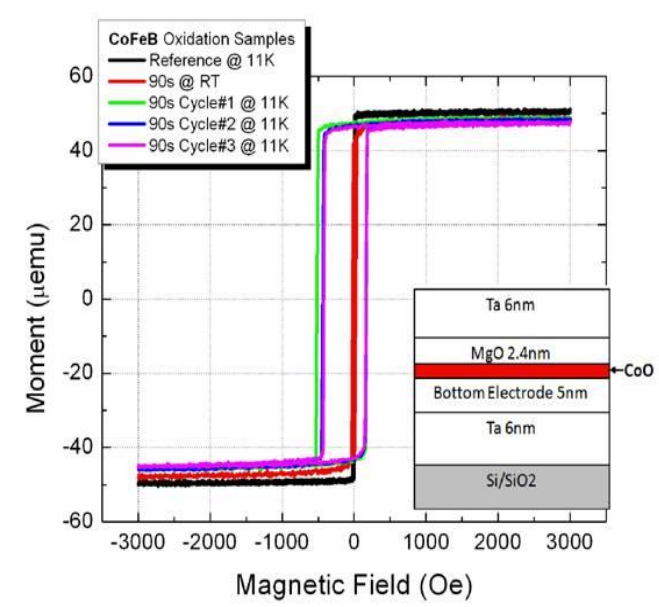

(b)

Figure 5.1 RT and $11 \mathrm{~K}$ hysteresis loop measurement of the sample with (a) CoFe (b) CoFeB bottom electrode and both with 90 seconds MgO barrier oxidation. Also shown is the corresponding reference sample (in black) with the same structure as the oxidized samples in (a) and (b) except no plasma oxidation of the Mg metal layer. The inset of (b) shows the general structure of the partial MTJ stack use in this study.

In Figure 5.1(a), all three loops of the reference sample in black color overlap with each other, and show very small coercivity (less than $20 \mathrm{Oe}$ ) when measured at $11 \mathrm{~K}$. This is in agreement with the typical value from the pure CoFe thin film with the same thickness. A similar small coercivity value was also observed from the oxidized sample measured at RT. However, the low temperature measurement of the oxidized sample shows significantly different hysteresis loops compared to the reference sample. First of all, the loop in green, which is the first of the three cycling loops, has a much larger coercivity compared with that of the reference sample. An irregular top left corner shape is also observed compared with the second and third loops shown in blue and magenta. 
The first irregular shaped loop with extremely large EB field and coercivity is known as due to the training effect of a typical EB system [61]. Based on the training effect, I define the Training Effect Ratio (TE) as follows to describe the magnitude of the training effect: $T E(\%)=\left(H_{t e}-H_{c}\right) / H_{c} \times 100 \%$, where $H_{t e}$ is defined as the coercivity measured from the first irregular hysteresis loop due to the training effect.

By cycling the hysteresis loop measurement, the training effect disappears after the first loop, and the loops go back to the regular shape and overlap with each other from the second cycle to the third. Based on the regular shaped loops the EB field is conventionally defined as $H_{e b}=-\left(H_{c}^{+}+H_{c}^{-}\right) / 2$, and the coercivity is defined as $H_{c}=\left(H_{c}^{+}-H_{c}^{-}\right) / 2$, where $H_{c}^{+}$and $H_{c}^{-}$are the positive and negative field values of the loop where saturation moments are zero. Another parameter, although not so easily observable from the hysteresis loop, is associated with the EB system and is called the uncompensated spin $(U C)$ density. In fact, the general understanding about the origin of $\mathrm{EB}$ is that it is the formation of uncompensated spins at the interface that causes the EB anisotropy [26]. Such uncompensated spins also cause the hysteresis loop to shift along the axis of magnetization, and is usually defined as $U C(\%)=\left(M_{S}^{+}-M_{S}^{-}\right) /\left(M_{S}^{+}+\right.$ $\left.M_{S}^{-}\right) \times 100 \%$, where $M_{S}^{+}$and $M_{S}^{-}$are the saturation magnetization of the samples at positive and negative field, respectively.

It was clearly observed that for the sample with the over-oxidized barrier, ignoring the first loop due to the training effect, the regular hysteresis loops still showed much larger coercivities compared to the reference samples. So the enhanced coercivity $H_{c}$, the training effect ratio $T E$, the $\mathrm{EB}$ field $H_{e b}$ and the uncompensated spin density $U C$ together make four good parameters for detecting the EB formation, hence the bottom 
FM electrode surface oxidation. Based on such an analysis, I concluded from Fig. 1 that the recipe of 90 seconds plasma oxidation of a $2.4 \mathrm{~nm} \mathrm{Mg}$ layer caused the overoxidation of the $\mathrm{Mg}$ layer, hence the surface oxidation of both $\mathrm{CoFe}$ and $\mathrm{CoFeB}$ electrodes for the two samples. This is due to the presence of the EB phenomenon identified with the training effect and enhanced coercivity of the hysteresis loop measurement at $11 \mathrm{~K}$. It is worth noting that the sample with the CoFe bottom electrode shows much larger values of those four detection parameters when compared to the sample with $\mathrm{CoFeB}$ electrode. This could be understood given the much higher Co concentration in the $\mathrm{CoFe}(95 \% \mathrm{Co})$ than $\mathrm{CoFeB}(60 \% \mathrm{Co})$ with the same film thickness and the same oxidation conditions.

To determine if the barrier layer is over-oxidized, i.e. the bottom electrode is partially oxidized, an apparent approach would be to compare the saturation magnetization value of the oxidized sample to the reference sample. In the case of a sample with a CoFe bottom electrode, if the surface of the CoFe layer gets oxidized due to the over-oxidation of the $\mathrm{MgO}$ barrier, the total magnetic moment of the CoFe layer will be reduced. Indeed, the reduced magnetic moment of the sample with over-oxidation of $\mathrm{MgO}$ layer (the red color loop in Fig. 1(a)), was observed when compared to the reference sample loop measured at RT (loop not shown). However, this parameter of reduced moment is not pursued in this study for two reasons. Firstly, in order to use the change of magnetic moment as a detection parameter, the sample size factor needs to be considered. The results could only be indirectly compared after the normalization of the surface area of different thin film samples. This makes the comparison less accurate and sensitive. Secondly, this parameter is not directly related to the EB phenomenon due to 
the bottom electrode surface oxidation. This makes the parameter less interesting in terms of physical significance.

\subsubsection{Sensitivity of the parameters}

For each type of FM electrode, one set of samples with the same structure specified in Figure 5.1(b) but with different $\mathrm{Mg}$ layer oxidation times were fabricated. Specifically, for samples with $\mathrm{CoFeB}$ electrode, $\mathrm{Mg}$ layer oxidation times were chosen at 90, 60, and 30 seconds; and with CoFe electrode, samples with Mg layer oxidation time 90, 60, 30, 20, 10, and 5 seconds were deposited for more detailed study. These two sets of samples, together with two reference samples, were measured at $11 \mathrm{~K}$ with $3 \mathrm{kOe}$ field cooling from RT. The hysteresis loop measurement was repeated three times in order to identify the training effect. Data from the second loop were taken for the calculation of the rest three parameters. 


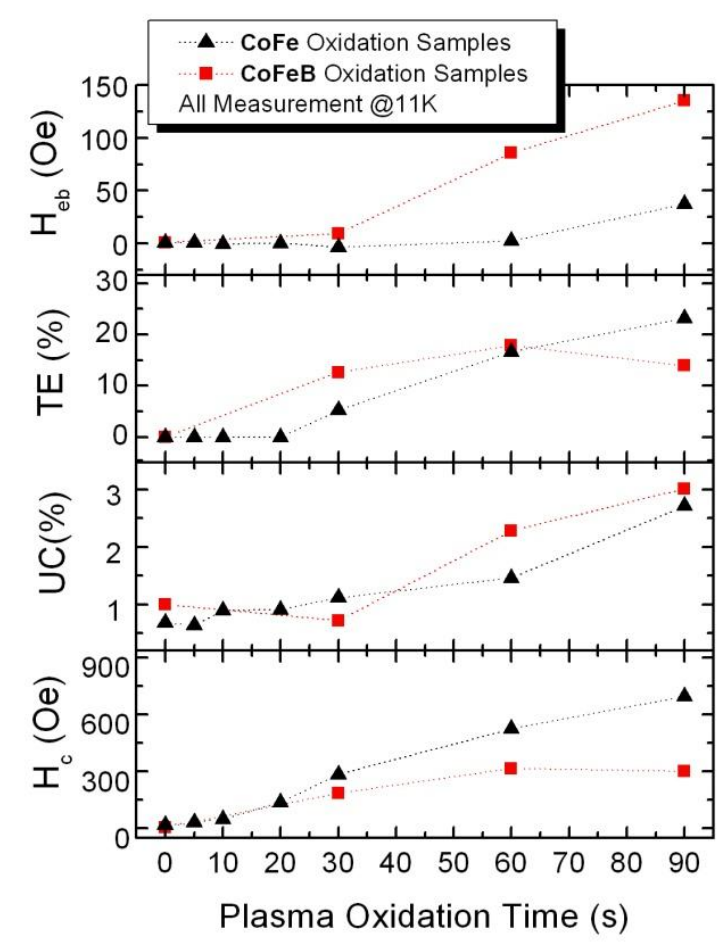

Figure 5.2 The oxidation time dependence of the four detection parameters $H_{e b}, H_{c}$, TE ratio and UC spin density. Points in black are from the samples with CoFe bottom electrodes and red points represent the data from samples with $\mathrm{CoFeB}$ electrodes. All measurements were done at $11 \mathrm{~K}$.

As shown in Figure 5.2, for the sample set with CoFe electrodes, as the oxidation time is decreased from 90 seconds, the $H_{e b}$ of corresponding samples starts to drop quickly and becomes almost zero for the sample with 30 seconds of oxidation. The training effect $T E$ starts to disappear on the sample with 20 seconds of oxidation. As to the third parameter, enhanced $U C$ compared to the reference sample is still observable even with only 10 seconds oxidation. The last parameter $H_{c}$ seems to be the most sensitive one, because the enhanced coercivity due to the EB could still be clearly observed even with only 5 seconds oxidation, when compared to the reference sample. 
This enhanced coercivity indicates that even with just 5 seconds oxidation, the $\mathrm{MgO}$ barrier was still over-oxidized, which led to the surface oxidation of the bottom CoFe electrode at the $\mathrm{MgO} / \mathrm{CoFe}$ interface and the formation of the $\mathrm{CoO} / \mathrm{CoFe} \mathrm{EB}$ system. The data from the oxidized samples with the $\mathrm{CoFeB}$ electrode presents a similar trend in terms of detection sensitivities among those four parameters in Figure 5.2. Study from both sets of samples showed that the enhanced coercivity $H_{c}$ is the most sensitive parameters to detect the existence of EB formation, followed by the uncompensated spin density $U C$ and the training effect $T E$. The EB field $H_{e b}$ seems to be least sensitive one among the four parameters. To measure these four parameters related to the EB effect, one can quickly examine the oxidation state of the $\mathrm{CoFeB}$ or other Co rich FM electrode adjacent to the oxide barriers. Consequently, the optimization of the tunnel barrier oxidation is feasible without characterizing the completed MTJ structure using a microfabrication process.

\subsubsection{Annealing effect on detection parameters}

For MTJs with an $\mathrm{MgO}$ barrier, it is necessary to anneal the samples in order to obtain crystallized $\mathrm{MgO}(001)$ for high spin filtering efficiency [23]. Previous study of $\mathrm{CoFeB} / \mathrm{MgO}$ bilayers using $\mathrm{x}$-ray photoemission spectroscopy (XPS) not only confirmed the process-dependent formation of $\mathrm{CoO}$ at the $\mathrm{CoFeB} / \mathrm{MgO}$ interface, which is similar to our findings using this over-oxidation detection method, but also unveiled the reduction of such oxidation during the vacuum annealing process [60]. To verify the existence of similar oxidation reduction in our sample structures and further study the properties of 
the four detection parameters after heat treatment, the sample set with $\mathrm{CoFeB}$ bottom electrodes, including both the reference sample and the oxidized samples, was annealed for one hour at two different temperatures $200^{\circ} \mathrm{C}$ and $350^{\circ} \mathrm{C}$, respectively. The same magnetic measurements were performed on the annealed samples as on the as-deposited ones, and the results are summarized in Figure 5.3.

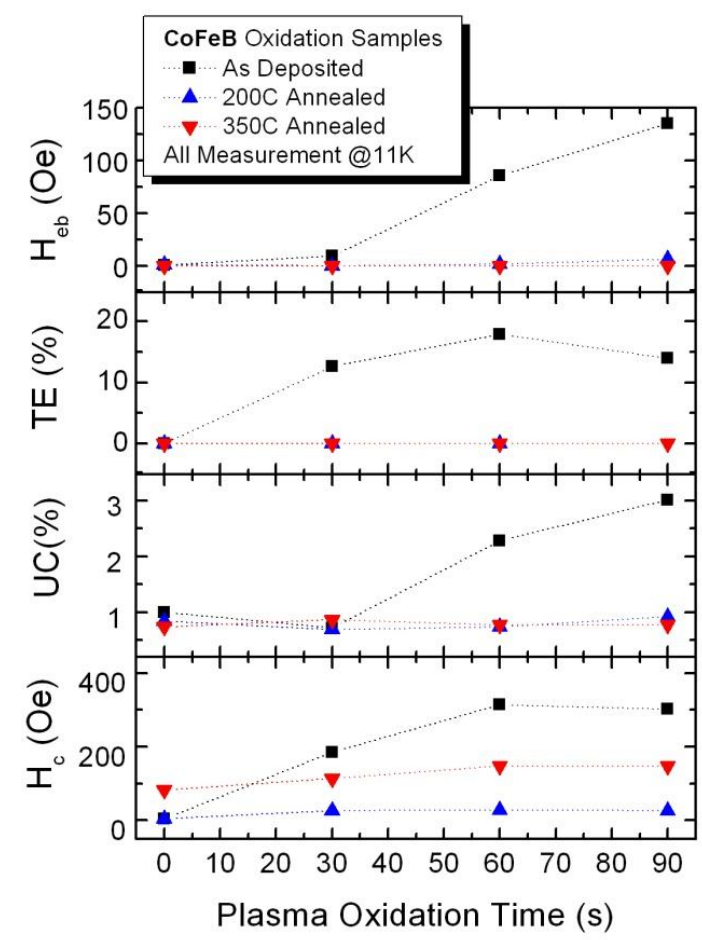

Figure 5.3 The annealing effect on the four detection parameters for sample sets with CoFeB bottom electrode. Data points in black are from the as-deposited samples, and blue and red points represent the data from samples annealing at $200^{\circ} \mathrm{C}$ and $350^{\circ} \mathrm{C}$, respectively. All measurement was done at 11 K.

Figure 5.3 shows that after the annealing treatment both at $200^{\circ} \mathrm{C}$ and $350^{\circ} \mathrm{C}$, except for the $H_{c}$ values, all other parameters become almost the same as those of the reference 
sample. The drop of $H_{e b}, T E$ and $U C$ values serve as a clear indicator of the reduced surface oxidation state of the bottom $\mathrm{CoFeB}$ electrode after the annealing. This is because during the annealing process, the oxygen atoms at the $\mathrm{CoFeB} / \mathrm{MgO}$ interface may diffuse back to the $\mathrm{MgO}$ barrier, and the decrease in the amount of $\mathrm{CoO}$ reduces the $\mathrm{CoO} / \mathrm{CoFeB}$ EB effect. This result is consistent with previous studies using XPS and similar magnetometry measurements $[60,62]$. One thing worth noting is the increase in the $H_{c}$ values of the samples annealed at $350^{\circ} \mathrm{C}$ compared to the results at $200^{\circ} \mathrm{C}$. The enhanced $H_{c}$ at $350^{\circ} \mathrm{C}$ is probably due to the crystallization of the CoFe films. However, it obscures the annealing effect on the underlying EB coupling and the oxidation state at the interface.

To interpret the $H_{c}$ data in a different manner, the change in $H_{c}$ was normalized by dividing the $H_{c}$ of each sample by its corresponding RT value. In Figure 5.4, the normalized $H_{c}$ data at different conditions shows clearly that the CoFeB surface oxidation is not completely reversed after the one hour annealing at $200^{\circ} \mathrm{C}$, and the EB coupling still exists in all of the oxidized samples. However, those oxidized samples with $350^{\circ} \mathrm{C}$ and $1 \mathrm{hr}$ annealing show almost identical $H_{c}$ values to the reference sample, indicating the disappearance of $\mathrm{EB}$ and $\mathrm{CoFeB}$ surface oxidation. This is because almost all of the oxygen atoms were absorbed by the $\mathrm{MgO}$ barrier after the higher temperature anneal. This conclusion is consistent with the annealing study of MTJs with $\mathrm{MgO}$ barriers. It was shown in that study that within certain temperature range, higher temperature annealing usually yields better TMR performances [63]. Again, this annealing effect study confirms that among the four detection parameters, the enhanced 
coercivity $H_{c}$ is the most sensitive one to detect the bottom electrode oxidation in the MTJ fabrication process.

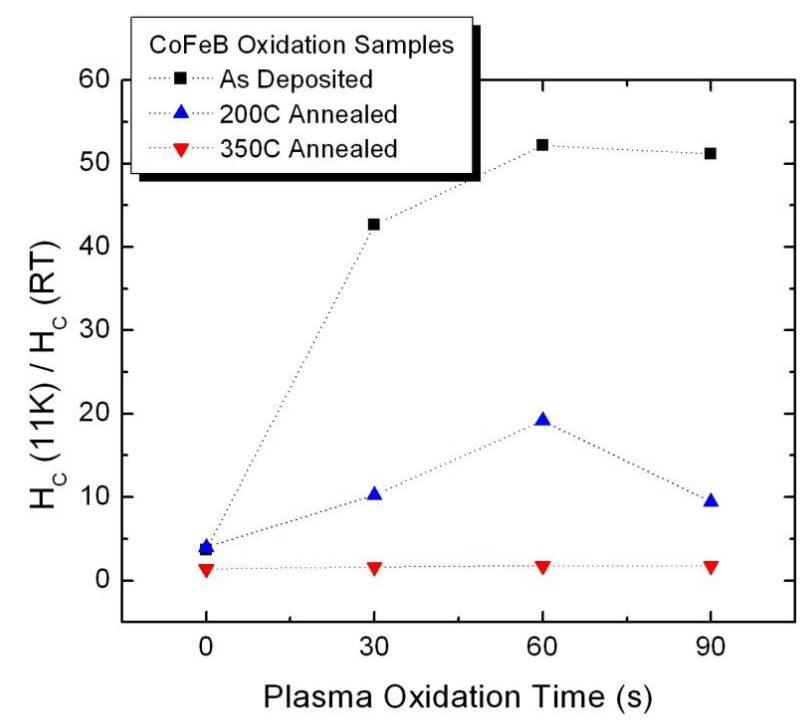

Figure 5.4 The normalized data of $\mathbf{H}_{\mathrm{c}}$ including the results after annealing for $1 \mathrm{hr}$ at two different temperatures $\left(200^{\circ} \mathrm{C}\right.$ and $\left.350^{\circ} \mathrm{C}\right)$, as well as the as-deposited data. The normalization was done by dividing the absolute values of $\mathrm{H}_{\mathrm{c}}$ by the corresponding values at RT.

\subsection{Summary}

In summary, a simple method of detecting oxidation of the bottom FM electrode was developed without going through the whole MTJ stack deposition and microfabrication process. This method could be used as a generic detection method and could be useful to quickly explore the oxidation process window of various barrier materials regardless of their types. Four different oxidation detection parameters were identified, among which 
the enhanced coercivity due to the exchange bias formation is most sensitive. By studying the annealing effect on these four parameters, the oxygen restoration to the barrier material with sufficient annealing treatment was confirmed, which is consistent with the results from previous studies with other more sophisticated experiments. 


\section{Interface engineering: effects of target bias voltage on magnetic tunnel junctions (MTJs)}

\subsection{Introduction}

For MTJ thin film stacks with layer thicknesses on the order of nanometers, it is well know that the layer surface roughness and intermixing between adjacent layers are among the key problems not only preventing the successfully switching of the free layer, but also resulting in reduced tunneling magnetoresistance (TMR) performance and unfavorable resistance-area (RA) product values. Others have studied the effects of deposition rate on the properties of single films [64] and multilayers $[65,66]$ to understand the dependence of film morphology on deposition rate, in hopes of reducing surface roughness and interlayer mixing for optimized growth conditions. Here, using a different approach, the effects of bias voltage (i.e. different adatom energies during deposition) on MTJ properties are studied via the investigation of the offset field (the distance of the loop center from the zero field origin) of the free layer due to its Néel coupling with the pinned layer, as well as the examination of the TMR performance. An optimization strategy based on the study to reduce both film surface roughness and intermixing is proposed. This study also demonstrates the BTIBD's capability of precise control over thin film growth and interface engineering at the atomic level. 


\subsection{Experiments}

As shown in Figure 3.1 of section 3.1.1, there're are two identical ion source guns installed in the BTIBD system, one for the main deposition and the other for ion beam assisted deposition and ion beam milling. In the work presented in this chapter, only the main deposition gun was used for fabricating all the MTJ samples studied here.

MTJ samples were deposited on clean thermally oxidized Si substrates, and the substrates were cleaned using the procedure described in section 3.2. The typical MTJ stack was $\mathrm{Ta}(5) / \mathrm{CoFe}(3) / \mathrm{FeMn}(6) / \mathrm{CoFe}(3) / \mathrm{AlO}_{\mathrm{x}}(2) / \mathrm{CoFe}(3) / \mathrm{Ta}(5)$ (all units in $\left.\mathrm{nm}\right)$, with the exchange bias trilayers described in chapter $\mathbf{4}$ being applied. The base pressure was better than $2 \times 10^{-7}$ Torr, and the working pressure was $7 \times 10^{-4}$ Torr during the deposition. An in-plane 50 Oe field was applied during the deposition of CoFe and FeMn layers to set the magnetic easy axis. A natural oxidation method was used to form the $\mathrm{AlO}_{\mathrm{x}}$ barrier, and the oxidation process was carried out in multiple steps to produce a better quality oxide barrier as suggested in Ref. [67]. For instance, a nominal thickness of $5 \AA$ Al was first deposited and naturally oxidized. This process was repeated four times to form the total $2 \mathrm{~nm} \mathrm{AlO}_{\mathrm{x}}$ barrier. The first $5 \AA \mathrm{Al}$ layer was oxidized in $500 \mathrm{mT}$ Torr $\mathrm{O}_{2}$ for 5 minutes and the next three $\mathrm{Al}$ layers were oxidized in 1 Torr $\mathrm{O}_{2}$ for 10 minutes. All of the oxidation steps were carried out by first transferring the wafer from the processing chamber to the transport chamber in the middle then introducing oxygen into the chamber. 


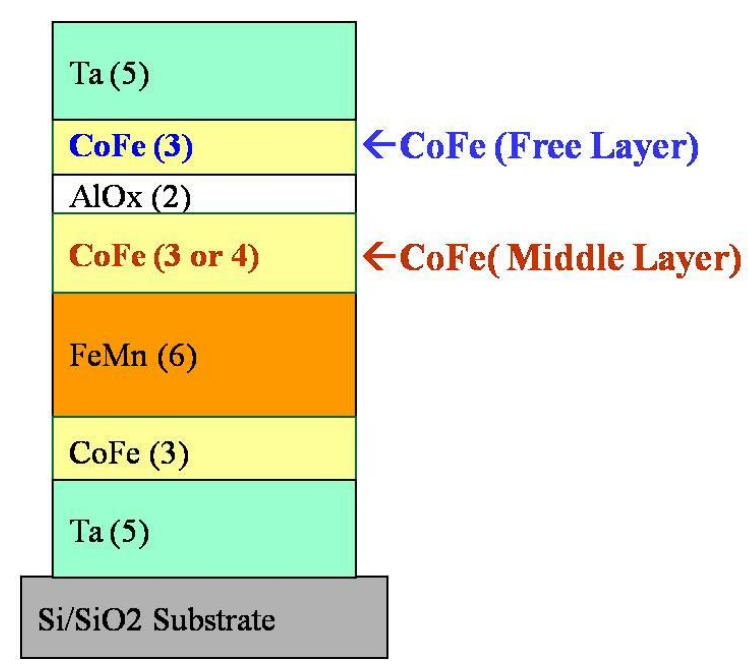

Figure 6.1 Schematic illustration of the typical MTJ stack

To study the effects of bias voltage on the MTJ properties, different bias voltages were applied for the deposition of the $\mathrm{CoFe}$ free layer on top of the $\mathrm{AlO}_{\mathrm{x}}$ barrier, and on this layer only in order to simply the investigation. Deposition rates were calculated from the film thicknesses measured by atomic force microscopy (AFM), and the deposition time was adjusted correspondingly so that the top CoFe free layer thickness was kept the same for different target bias voltage used. To create the unidirectional exchange anisotropy, the samples were quickly field-cooled from $250^{\circ} \mathrm{C}$ to room temperature in a 3 kOe external field and protected by a constant-flowing forming gas by using our forming gas furnace. Magnetic properties were measured with a Quantum Design PPMS-6000. The TMR data was obtained by the current-in-plane tunneling (CIPT) technique [68] of un-patterned blanket films. In this study, two sets of samples were made to investigate the target bias voltage effects on MTJ properties. The first set consisted of three samples with the same typical structure mentioned previously. All of the layers of the three samples were deposited with a bias voltage $600 \mathrm{~V}$ except for each of the top CoFe free 
layers, where the bias voltages of $300 \mathrm{~V}, 600 \mathrm{~V}$, and $900 \mathrm{~V}$ were used respectively. For the second set, all conditions were the same as the first except for the thickness of the middle CoFe layer. Instead of $3 \mathrm{~nm}$ used in the first set, I increased the thickness to $4 \mathrm{~nm}$ for all three samples in the second set.

\subsection{Results and discussions}

Figure 6.2 shows the simulated TMR data from the CIPT measurement of a MTJ sample with the nominal pre-oxidized Al layer thickness of $2 \mathrm{~nm}$ (the second sample in the second set). All the layers were deposited with a target bias voltage of $600 \mathrm{~V}$ and the middle CoFe layer thickness of $4 \mathrm{~nm}$. The TMR value of this sample is close to $20 \%$. This TMR value is not a record high value, partly because the CoFe target used has a composition of $\mathrm{Co}_{95} \mathrm{Fe}_{5}$, which was found not to be the optimal composition for producing high TMR [69].

Regarding the interlayer magnetic coupling between the two ferromagnetic electrodes of MTJ, previous studies have shown that two separate effects tend to produce extraneous magnetic field in the plane of free layer: magnetostatic coupling due to uncompensated poles near the sample edges and the Néel coupling due to interfacial roughness [66]. For un-patterned samples in our case, the magnetostatic coupling is negligible. Another requirement before we can assume that the Néel coupling is the only magnetic coupling affecting the free layer switching is that the tunnel barrier of the MTJ must be pinhole free. Without this condition, we cannot separate the indirect Néel coupling from the direct magnetic coupling through the pinholes. 


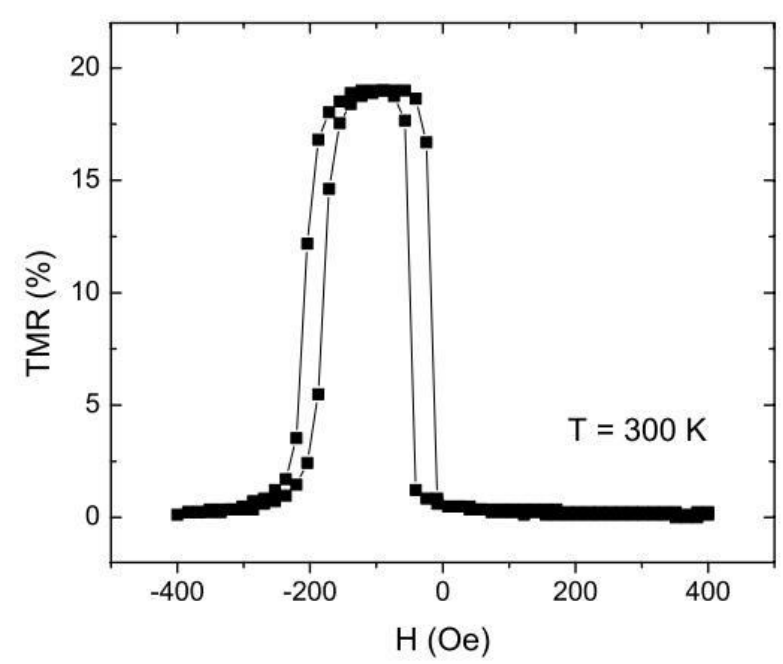

Figure 6.2 Representative TMR result from the CIPT measurement. (Adapted from Ref. [70])

To prove that the MTJ is pinhole free, I measured the temperature dependence of the $\mathrm{M}(\mathrm{H})$ hysteresis loop center shifts of both the free and pinned layers of all the MTJ samples, and compared the loop center shifts at different temperatures to the value at 300 K. The shift for a layer is defined as the magnetic field of the center of its minor $\mathrm{M}(\mathrm{H})$ loop that was shifted from zero field by exchange bias (for the pinned layer) or by the coupling with the pinned layer (for the free layer). Figure 6.3 shows a representative measurement for the second sample in the second set with a free layer deposited at $600 \mathrm{~V}$ and a $4 \mathrm{~nm}$ middle CoFe layer. We can see in Figure 6.3 an increasing loop center shift of the pinned layer as the temperature decreases. This is because the pinning strength of the antiferromagnetic FeMn gets larger at lower temperatures, which means the exchange bias of the pinned layer increases as the temperature decreases. However, the loop center shifts of the free layer remain almost constant regardless of the temperature change. If there were pinholes, the free layer would be directly coupled with the pinned layer. Then, 
as the pinned layer loop shifts with temperature, the direct magnetic coupling would force the free layer loop to shift as well. The result of the temperature dependent measurement suggests that, according to Ref. [71], the $\mathrm{AlO}_{\mathrm{x}}$ barrier in this sample is free of pinholes. Similar results indicating pinhole-free-barriers were also obtained for the other five samples in our investigation.

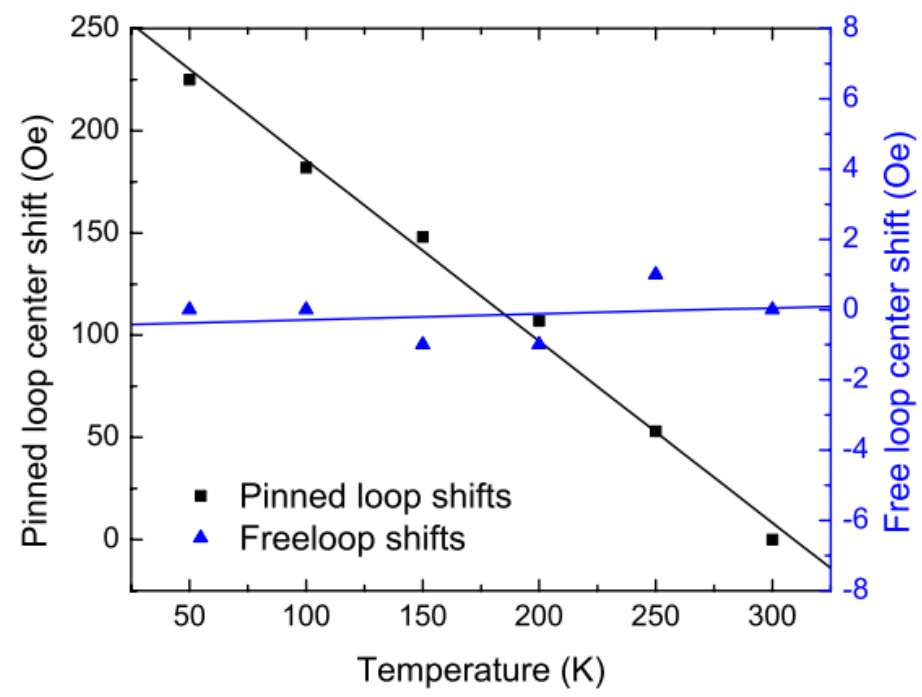

Figure 6.3 Representative loop center shifts of both free and pinned layers at different temperatures. (Adapted from Ref. [70])

According to Néel's model [66], Néel coupling is closely related to the film surface and interface morphology. In this model, a sinusoidal roughness profile is assumed, and the Néel coupling field is given by

$$
H_{N}=\frac{\pi^{2}}{\sqrt{2}}\left(\frac{h^{2}}{\lambda t_{F}}\right) M_{P} e^{\left(-\frac{2 \pi \sqrt{2} t_{s}}{\lambda}\right)}
$$


where $\lambda$ and $h$ are the amplitude and wavelength of the roughness profile, $t_{F}$ and $t_{s}$ are the thickness of the free layer and the barrier respectively as shown in Figure 6.4 , and $M_{P}$ is the magnetization of the pinned layer.

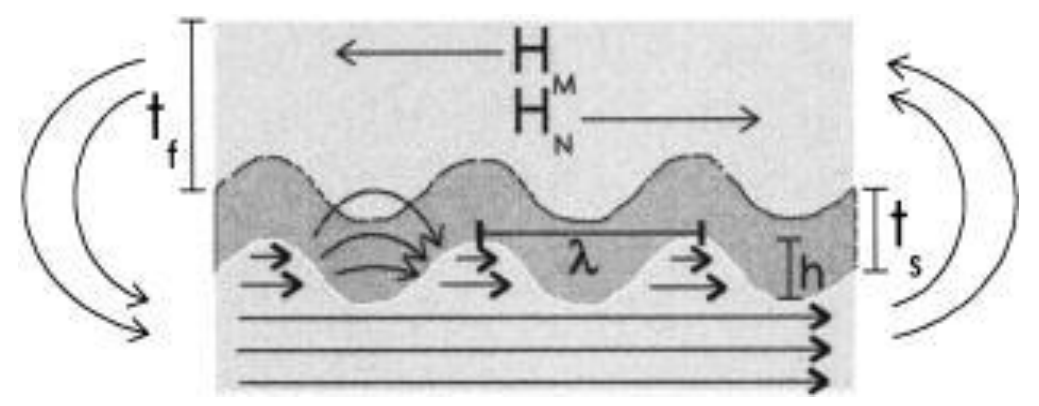

Figure 6.4 Schematic illustration of Néel coupling model. As the two dominant interlayer coupling mechanisms, $H_{M}$ and $H_{N}$ represent the magnetostatic coupling and the Néel coupling respectively. Here in our case of un-patterned films, $H_{M}$ is negligible and only $H_{N}$ needs to be considered. (Adapted from Ref. [66])

Since Néel coupling has been proven by the temperature dependent hysteresis loop measurement to be the only effect responsible for the free layer hysteresis loop center shift in our samples, we can extract the information about the interfacial mixing and film surface roughness by studying the Néel coupling field of the free layer. Figure 6.5 shows the free layer loop center shifts at room temperature for the two sets of samples described at the end of section 6.2. First, we compare the results of the loop center shifts between the two sets. The second set, with thicker middle CoFe layer (i.e. thicker pinned ferromagnetic layer with larger $M_{P}$ ), always shows a higher Néel coupling offset field for the free layer when compared to corresponding samples with the same bias voltage in the first set. This is qualitatively consistent with the Néel model presented in eq. 6.1. 
Additionally, Figure 6.5 shows that within each sample set, the Néel coupling field is stronger with increased target bias voltage for the free layer deposition.

There are two possible competing factors when considering the impact of high energy adatoms on the film morphology. One is that the high energy adatom of the free layer, when hitting the barrier surface, could redistribute the surface clusters and flatten the barrier surface. This would effectively increase the barrier thickness, so that the coupling field would be reduced according to Néel's model. Alternatively, adatoms with high energy could cause significant interlayer mixing between the top free CoFe layer and the barrier layer underneath, which effectively makes a rougher interface compared to the barrier surface before the free layer deposition. This leads to a stronger Néel coupling and therefore a larger loop center shift of the free layer hysteresis loop. From the above result in Figure 6.5, the stronger Néel coupling with increased bias voltage indicates at least the existence of an intermixing effect and it should be dominant even if the barrier surface is flattened during the free layer deposition. 


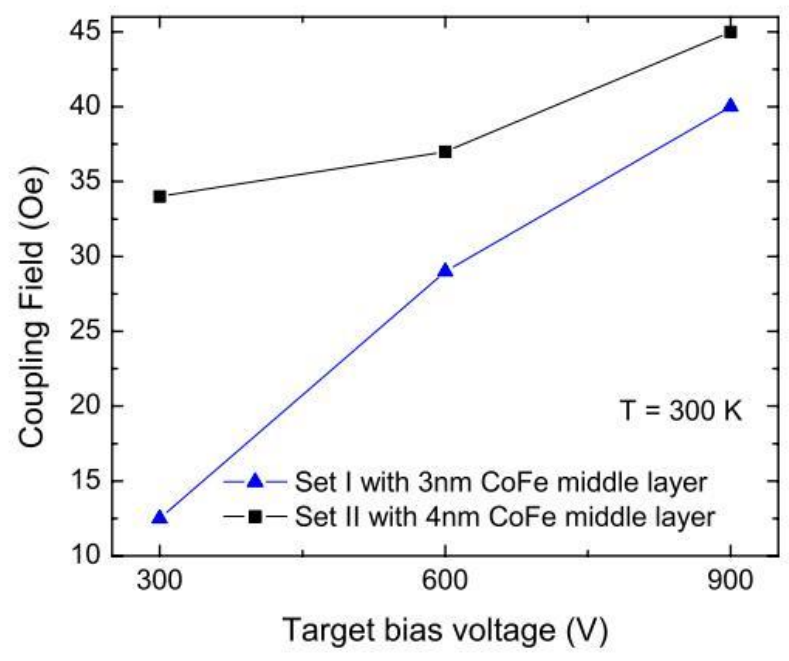

Figure 6.5 The dependence of free layer loop center shift on the target bias voltage used for the free layer deposition. (Adapted from Ref. [70])

To better study the two possible factors mentioned above due to the bias voltage effect, I also investigated the dependence of the TMR and RA values on the bias voltage. Figure 6.6 shows the TMR and RA values of the second sample set with different bias voltages applied for free layer deposition. The higher adatom energy causes more interlayer mixing between the tunnel barrier and the free layer. This intermixing could smear the thin barrier, causing stronger electron scattering at the interface, so a lower TMR value would be expected. Also such intermixing can effectively make the barrier thinner, so the RA value would decrease with higher bias voltage too. On the other hand, due to the impact of the same high energy adatom at the beginning stage of the deposition, the possible barrier surface flattening could effectively make the barrier thicker and sharper, so larger RA and TMR values would be expected. As shown in Figure 6.6, both RA and TMR values are maximized at $600 \mathrm{~V}$ compared with the values 
at $300 \mathrm{~V}$ and $900 \mathrm{~V}$. Such result indicates that both the factors of intermixing and surface flattening coexist and compete with each other. From $300 \mathrm{~V}$ to $600 \mathrm{~V}$, the initial barrier surface flattening due to higher adatom energy helps to get higher RA and TMR values. From $600 \mathrm{~V}$ to $900 \mathrm{~V}$, the high adatom energy makes the interlayer mixing the dominant effect, causing both reduced RA and TMR values.

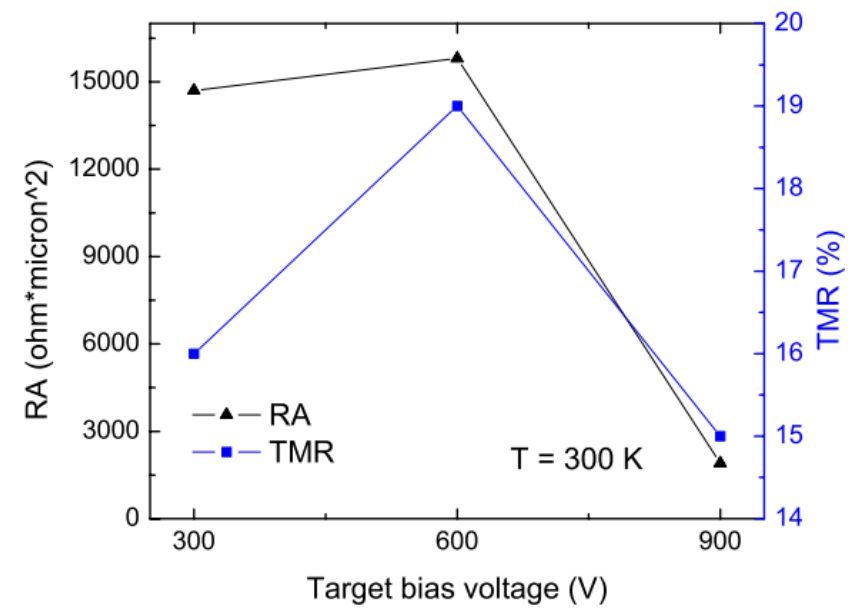

Figure 6.6 The dependence of TMR and RA values on the target bias voltage applied for the free layer deposition. (Adapted from Ref. [70])

Based on the above analysis, in order to improve the TMR performance of the MTJs, high adatom energy should be used for the deposition of the bottom ferromagnetic layer before the barrier deposition. This creates a smooth seeding surface for the following barrier layer deposition, which is particularly important considering the ultrathin barrier on the order of a just few nanometers. For the top ferromagnetic electrode deposition after the barrier layer, optimized intermediate adatom energy could prevent intermixing while at the same time flatten the barrier surface for best spin-polarized electron 
tunneling. To further optimize the film quality, during the deposition of each single layer film within the multilayer structure, low initial growth energy should be used to prevent intermixing at the interface. While In the late stage of the film layer deposition, relatively high adatom energy should be applied till the completion of the whole layer deposition to create a flatter seeding surface for the following layer. For applications of MTJs that require minimum Néel coupling, a low bias voltage would be preferred for the top ferromagnetic deposition.

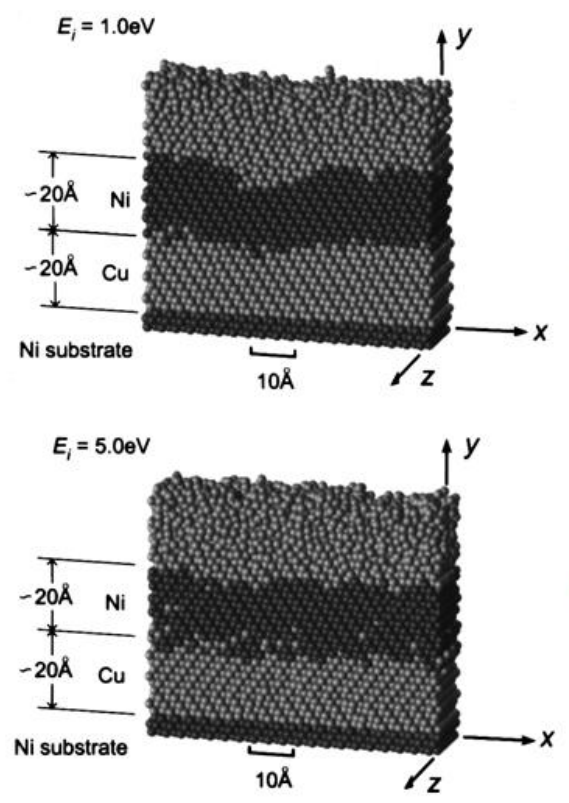

(a)

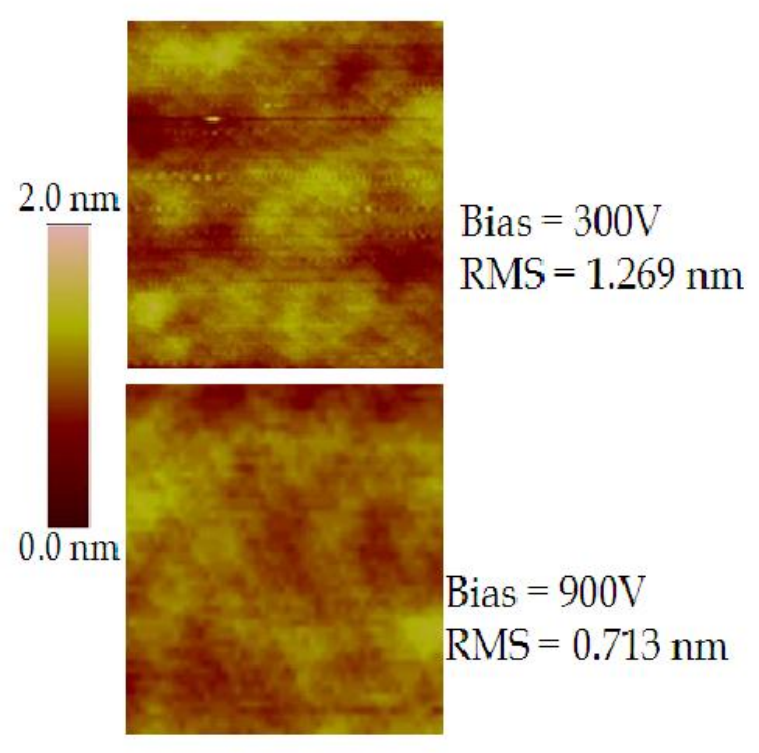

(b)

Figure 6.7 (a) Atomic configurations of $\mathrm{Cu} / \mathrm{Ni} / \mathrm{Cu}$ multilayers deposited with different incident adatom energies simulated by molecular dynamics. (Adapted from Ref. [41]) (b) AFM images of single CoFe layer deposited by BTIBD with different bias voltages. 
The above study focused mainly on the impact of the bias voltage on the interfacial properties, pointing out two main factors affecting the interfacial profile and properties of multilayer structures. Basically, if the adatom energy is too high, which is associated with high bias voltage, it will render films with relatively flat surface morphology. However, it will also cause a strong interlayer mixing. On the other hand, relatively low adatom energy, which is associated with low bias voltage, will create a relatively rough surface, but with much reduced interlayer mixing. These conclusions are consistent with the simulated atomic configuration of $\mathrm{Cu} / \mathrm{Ni} / \mathrm{Cu}$ multilayers deposited with different incident adatom energies by molecular dynamics [41] as shown in Figure 6.7 (a). In Figure 6.7 (b), are presented the atomic force microscopy (AFM) images of two single CoFe layers deposited by the BTIBD with the same nominal thickness of $10 \mathrm{~nm}$ but with different bias voltages of $300 \mathrm{~V}$ and $900 \mathrm{~V}$ respectively. The surface roughness is indicated by the root mean square (RMS) values obtained from the AFM data that were $1.269 \mathrm{~nm}$ and $0.713 \mathrm{~nm}$ for the $300 \mathrm{~V}$ and $900 \mathrm{~V}$ sample respectively. Again, this is consistent with the conclusion of the simulation that higher adatom energy deposition yields the smoother surface morphology.

\subsection{Summary}

In this chapter, I demonstrated the capability of depositing MTJs with $\mathrm{AlO}_{\mathrm{x}}$ barriers by the BTIBD system. The low energy source and target voltage bias as well as the unique design of the BTIBD make it well suited for multilayer film deposition for MTJs, which require atomic level interfacial engineering. The study of bias voltage effects on 
the interfacial and the film surface properties via Néel coupling facilitated our understanding of the adatom energy impact on both the film stack and the interface properties. A strategy, based on our study, for the best performance of MTJs would be to use high bias voltage for the bottom ferromagnetic electrode deposition to get a smooth seeding surface, and using optimized intermediate bias level for the free layer to get high TMR value. For applications that require minimum Néel coupling, low bias voltage would be preferred for the free layer deposition. 


\section{Magnetic tunnel junctions with MgO tunnel barrier by bias target ion beam deposition}

\subsection{Introduction}

When large tunneling magnetoresistance (TMR) over $10 \%$ at room temperature (RT) was first experimentally achieved in 1995, the tunnel barrier material used in the magnetic tunnel junction (MTJ) was $\mathrm{Al}_{2} \mathrm{O}_{3}[1,2]$. This discovery triggered great research interest in the subject. Almost 10 years later in 2004, the highest TMR record of $70 \%$ was obtained on $\mathrm{Al}_{2} \mathrm{O}_{3}$ based MTJs [19]. This record was achieved by optimizing the conditions for fabricating the $\mathrm{Al}_{2} \mathrm{O}_{3}$ and replacing the conventional $\mathrm{Fe}$ or $\mathrm{CoFe}$ ferromagnetic electrode with the new material candidate $\mathrm{CoFeB}$. The enhanced TMR performance was primarily due to the amorphous nature of the as-deposited CoFeB layer and the resulting smooth interface between the $\mathrm{CoFeB}$ electrode and $\mathrm{Al}_{2} \mathrm{O}_{3}$ barrier layer [72]. However, the 70\% record TMR from the $\mathrm{Al}_{2} \mathrm{O}_{3}$ based MTJs is still not high enough for many spintronic device applications. For example, next generation high density magnetic random access memory (MRAM) requires a TMR value higher than $150 \%$ [27]. Also, the resistance-area product of $\mathrm{Al}_{2} \mathrm{O}_{3}$ based MTJ is usually in the range of several thousand $\Omega \mu \mathrm{m}^{2}$, but for the spin torque transfer (STT) and next generation ultrahighdensity hard disk drive (HDD) application, the RA product value needs to be as low as several $\Omega \mu \mathrm{m}^{2}$. Grandis reported the record low RA value of $8.4 \Omega-\mu \mathrm{m}^{2}$ but at the cost of a low TMR ratio of only $9.5 \%$ [73].

$\mathrm{Al}_{2} \mathrm{O}_{3}$-based MTJs cannot meet the requirement for future technological applications, and the search continued for new material candidates to push the technology 
to the next level. In 2001, it was predicted by Butler et al. that the epitaxial MTJs with a crystalline $\mathrm{MgO}$ tunnel barrier would have a TMR ratio over 1000\% [20]. This prediction was partially confirmed in 2004 by the reported $200 \%$ TMR value from MgO based MTJs from two different groups independently [21, 22]. This giant TMR is due to the high spin polarized tunneling through the $\mathrm{Fe}(001) / \mathrm{MgO}(001)$ interface [20].

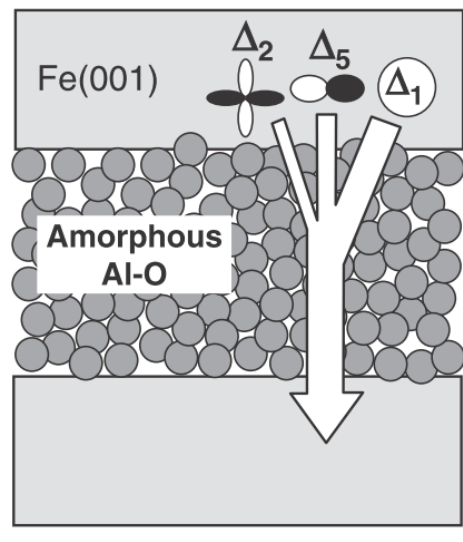

(a)

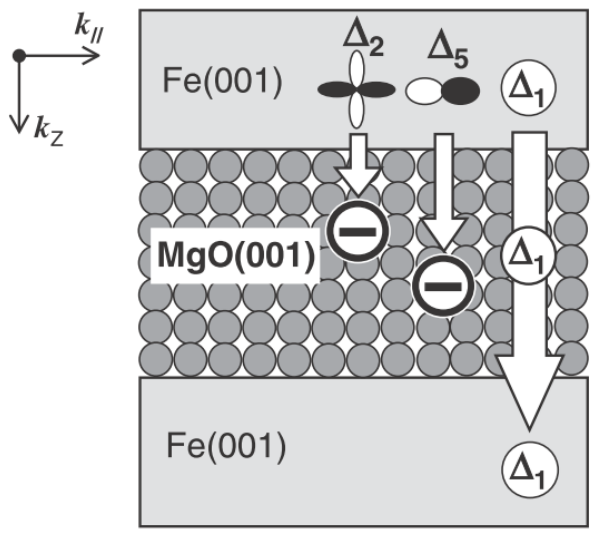

(b)

Figure 7.1 Schematic illustrations of spin polarized electron tunneling through (a) an amorphous $\mathrm{Al}_{2} \mathrm{O}_{3}$ barrier and (b) a crystalline $\mathrm{MgO}(001)$ barrier, reproduced from Ref. [27].

To further understand the different TMR performance between the $\mathrm{Al}_{2} \mathrm{O}_{3}$ and $\mathrm{MgO}$ based MTJs, here we compare the spin polarized tunneling process inside each structure as shown in Figure 7.1. Here we take $\mathrm{Fe}(001)$ as an example of the ferromagnetic (FM) electrode. Inside the $3 \mathrm{~d} \mathrm{FM} \mathrm{Fe(001),} \mathrm{there} \mathrm{are} \mathrm{spin} \mathrm{polarized} \mathrm{electrons} \mathrm{in} \mathrm{various} \mathrm{Bloch}$ states with different orbital symmetries. For example, the totally symmetric $\Delta_{1}$ Block state usually has a large positive spin polarization at the Fermi energy level $\mathrm{E}_{\mathrm{F}}$. Other states with lower symmetry like $\Delta_{2}$ have negative spin polarization at $\mathrm{E}_{\mathrm{F}}$ but also smaller 
polarization magnitude. This is why the net spin polarization of the 3d FM like Fe is still positive [27]. In the case of an amorphous $\mathrm{Al}_{2} \mathrm{O}_{3}$ barrier, there is no crystallographic symmetry in the tunnel barrier and at the barrier/electrode interfaces. So electrons in different Block state all have finite tunneling probabilities. The $\Delta_{1}$ Block state with larger positive spin polarization is considered to have larger tunneling probability than other states $[74,75]$. However, the total spin polarization gets much reduced after tunneling because of the negative spin polarized states like $\Delta_{2}$, which also has a finite tunneling probability due to the amorphous nature of the $\mathrm{Al}_{2} \mathrm{O}_{3}$ barrier. While in the case of a crystalline $\mathrm{MgO}(001)$ tunnel barrier, due to the crystallographic symmetry in the tunnel barrier and at the barrier/electrode interfaces, the $\Delta_{1}$ Block state continues to dominate with high spin polarization and large tunneling probability. However, the tunneling probability for the $\Delta_{2}$ state with a negative spin polarization decays quickly to zero with a $\mathrm{MgO}$ thickness of less than 5 monolayers [20]. So there are no negatively polarized states from $\Delta_{2}$ tunneling through the barrier. This explains the tunneling difference between the amorphous $\mathrm{Al}_{2} \mathrm{O}_{3}$ and crystalline $\mathrm{MgO}$ barrier, and the resulting large TMR ratio observed in the MTJs with $\mathrm{MgO}(001)$ crystalline tunnel barriers.

The most common fabrication technique used to grow good quality $\mathrm{MgO}$ barrier is using Radio Frequency Magnetron Sputtering directly from an $\mathrm{MgO}$ target [21]. Our BTIBD system, however, can only sputter from a conducting metal target because of the bias sputtering mechanism. In order to form the $\mathrm{MgO}$ barrier with a $\mathrm{Mg}$ metal deposition, several different approaches could be applied, including reactive sputtering in an oxygen environment and post-deposition plasma/natural oxidation. Among these techniques, the post-deposition natural oxidation proves to be the most suitable method, resulting in a 
high quality $\mathrm{MgO}$ barrier and smooth barrier/electrode interfaces [76]. In this chapter, we set the benchmark of $\mathrm{MgO}$ based MTJs by fabricating a multilayer stack using our Bias Target Ion Beam Deposition (BTIBD) system. The MgO barrier was formed by the postdeposition natural oxidation method. By tuning the MTJ structure parameters like the thickness of the tunnel barrier or the FM electrode, as well as the post-deposition annealing conditions, we were able to improve the magnetic properties of MTJs.

\subsection{Experiments}

\subsubsection{Single barrier MgO-MTJs}

The typical $\mathrm{MgO}$ based MTJs have the following structure: Ta6/V2/Ta6/CoFe6/FeMn10/CoFe1.5/CoFeB5/MgO1.8/CoFeB5/Ta6 (all units in nm). All the metal layers were deposited by sputtering the corresponding metal targets in the BTIBD system. The compositions of those alloys are $\mathrm{Co}_{95} \mathrm{Fe}_{5}, \mathrm{Fe}_{50} \mathrm{Mn}_{50}$ and $\mathrm{Co}_{60} \mathrm{Fe}_{20} \mathrm{~B}_{20}$, respectively. Instead of a single Ta $(6 \mathrm{~nm})$ seed layer, a Ta6/V2/Ta6 multilayer structure was chosen as the seed layer to create a smaller resistance for the bottom part of the MTJ stack. This is primarily due to the resistance requirement for the current in plane tunneling (CIPT) technique, which is a novel method to quickly measure the TMR ratio of the MTJs blanket film without going through the complicated microfabrication process [68]. Also, instead of simply depositing a thicker layer of Ta film with equivalent resistance, the Ta6/V2/Ta6 multilayer structure was used due to the improved surface smoothness. For thicker Ta films with thicknesses larger than $10 \mathrm{~nm}$, the partial crystallization of the Ta polycrystalline film become further enhanced, and this resulted 
in a much rougher surface morphology compared to the $\mathrm{Ta} / \mathrm{V} / \mathrm{Ta}$ multilayers examined by Atomic Force Microscopy (AFM).

For the exchange biased (EB) reference layer with fixed magnetization, the $\mathrm{CoFe} 6 / \mathrm{FeMn} 10 / \mathrm{CoFe} 1.5 / \mathrm{CoFeB} 5$ structure was used instead of the simple $\mathrm{FeMn} / \mathrm{CoFeB}$ bilayers. The reason to use $\mathrm{CoFeB}$ as the ferromagnetic (FM) electrode with a direct interface with the $\mathrm{MgO}$ barrier is due to the lattice matching between the two that allows high spin polarized tunneling as explained in section 7.1. However, due to the amorphous nature of the as-deposited $\mathrm{CoFeB}$, the EB coupling could not be established at the interfaces from either $\mathrm{CoFeB} / \mathrm{FeMn}$ or $\mathrm{FeMn} / \mathrm{CoFeB}$ structure [19]. Here I applied the $\mathrm{CoFe} / \mathrm{FeMn} / \mathrm{CoFe}$ trilayer structure with good $\mathrm{EB}$ coupling as developed in chapter 4 . When $5 \mathrm{~nm} \mathrm{CoFeB}$ was deposited directly onto the $1.5 \mathrm{~nm} \mathrm{CoFe}$, the magnetic measurements showed that $1.5 \mathrm{~nm} \mathrm{CoFe}$ was thick enough to ferromagnetically couple with the $5 \mathrm{~nm} \mathrm{CoFeB}$ layer, making the whole $\mathrm{CoFe} 6 / \mathrm{FeMn} 10 / \mathrm{CoFe} 1.5 / \mathrm{CoFeB} 5$ structure a good bottom electrode with fixed magnetization $\left(\mathrm{H}_{\mathrm{EB}} \sim 160 \mathrm{Oe}\right)$.

For the $\mathrm{MgO}$ tunnel barrier formation, post-deposition natural oxidation was chosen as the oxidation method. Specifically, after the metallic $\mathrm{Mg}$ layer deposition in the process module (PM) chamber of BTIBD, the samples were transferred to the middle transport module (TM) chamber, where pure oxygen was introduced to a certain controlled pressure for the natural oxidation process. It was found that when the thickness of the metallic $\mathrm{Mg}$ layer was larger than $1.2 \mathrm{~nm}$, it could not be fully oxidized even with very large oxygen pressure. As pointed out in the study of $\mathrm{Al}_{2} \mathrm{O}_{3}$ based MTJs, the leftover un-oxidized Al layer, even just a few monolayers, proved to be detrimental to the spinpolarized tunneling and greatly reduced the TMR values. That is why the two step natural 
oxidation method was developed to form the $\mathrm{Al}_{2} \mathrm{O}_{3}$ barrier with larger barrier thicknesses [73]. Here the two step natural oxidation method was applied to form the $\mathrm{MgO}$ barrier as well. Specifically, for any $\mathrm{MgO}$ barrier with thickness larger than $1.2 \mathrm{~nm}$, I first deposited the nominal $1.2 \mathrm{~nm} \mathrm{Mg}$ metal layer, and then the sample was transferred to the $\mathrm{TM}$ chamber for the first natural oxidation step. The oxygen pressure was controlled at 10 Torr and the oxidation time was kept to $10 \mathrm{~min}$ for the first $1.2 \mathrm{~nm}$ of $\mathrm{Mg}$. After the first oxidation step, the sample was transferred back to the PM chamber for the remaining $\mathrm{Mg}$ layer deposition. Then the sample was transferred back to the TM chamber again for the second natural oxidation step, with the oxygen pressure kept at 10 Torr and oxidation rate of $50 \mathrm{~s}$ per $\AA$ of $\mathrm{Mg}$ thickness. For example, in order to form a $1.8 \mathrm{~nm}$ thick $\mathrm{MgO}$ barrier, a $1.2 \mathrm{~nm} \mathrm{Mg}$ layer was deposited first and oxidized at 10 Torr for $10 \mathrm{~min}$. The sample was then transferred back to PM chamber for the remaining $0.6 \mathrm{~nm} \mathrm{Mg}$ deposition. The second oxidation step was carried out using 10 Torr oxygen pressure for $50 \times 6=300 \mathrm{~s}$.

Before characterization, the as-deposited full MTJ stacks were vacuum annealed to form the crystallized $\mathrm{MgO}$ and the $\mathrm{CoFeB} / \mathrm{MgO}$ interfaces. As explained in section 7.1, such crystallization is necessary for the high spin polarized tunneling across the $\mathrm{MgO}$ barrier and the $\mathrm{MgO} / \mathrm{CoFeB}$ interface. The base pressure of our home-built vacuum furnace was around $1 \times 10^{-6}$ Torr, which was proven to be low enough to prevent oxidation of the MTJs with $6 \mathrm{~nm}$ Ta capping layer. A small magnetic field $\sim 400$ Oe was provided for the annealing furnace with two permanent magnetic bricks. The magnetic field was arranged to be parallel to the easy axes of the FM electrode of the MTJs, which were set during the film deposition process with the applied magnetic field inside the PM chamber. As was mentioned earlier in this section, the TMR value was obtained by the CIPT 
technique that does not require the complicated patterning process of MTJ into small micron-size cell units [68].

\subsubsection{Double barrier MgO-MTJs}

Besides single barrier based MTJs, MTJs with double barriers were also fabricated. The basic structure of a double barrier magnetic tunnel junction (DBMTJ) is as follows: Substrate/Ta6/V2/Ta6/CoFe6/FeMn10/CoFe1.5/CoFeB5/MgO1.2/MgO1.2/CoFeB3/Mg

O1.2/MgO1.2/CoFe3/Ta1.5/CoFe6/FeMn10/Ta10 (all unites in nm). This DBMTJ material stack is also shown in the following Figure 7.2. There are two types of DBMTJs, depending on the relative orientation of the two EB pinned reference layers, with the two pinned layers either parallel or antiparallel to each other. Each configuration, as explained later in section 7.3.2, has its own advantages and disadvantages and is suited for different applications.

The fabrication of a DBMTJ with the two parallel pinned layers is relatively straightforward. After depositing all the layers on a thermally oxidized silicon substrate, the sample was put into the vacuum furnace for annealing at $400^{\circ} \mathrm{C}$ for $30 \mathrm{mins}$, with applied field around 400 Oe to create the EB coupling of both top and bottom pinned layers with the same pinning direction. However, to create antiparallel EB pinning directions for top and bottom pinned layers, the solution was more complicated. After the formation of the top $\mathrm{MgO}(2.4 \mathrm{~nm})$ barrier by the usual two-step natural oxidation , a thin layer of CoFe around $3 \mathrm{~nm}$ was deposited and then the stack was capped with Ta around $5 \mathrm{~nm}$. The vacuum was broken at this point and the partial structure was taken out of the 
processing chamber for annealing at $400^{\circ} \mathrm{C}$ for $30 \mathrm{~min}$ to set the $\mathrm{EB}$ pinning of the bottom pinned layers and to form two good crystallized $\mathrm{MgO}$ barriers above and below the shared CoFeB $(3 \mathrm{~nm})$ free layer. After the annealing treatment, the partial structure was put back into the BTIBD process chamber for further processing. The assist gun sitting on top of the process chamber was used to etch away part of the Ta capping layer by ion milling, and a nominal thickness of $1.5 \mathrm{~nm}$ Ta was left after the ion milling process. Then the rest of the top layers were deposited as shown in Figure 2, and an external field opposite to the direction of the bottom pinning was set during the processing of the top magnetic layers to create the EB coupling of the top pinned layers with the pinning direction antiparallel to the bottom pinned layers, so that DBMTJs with antiparallel pinned layers were created.

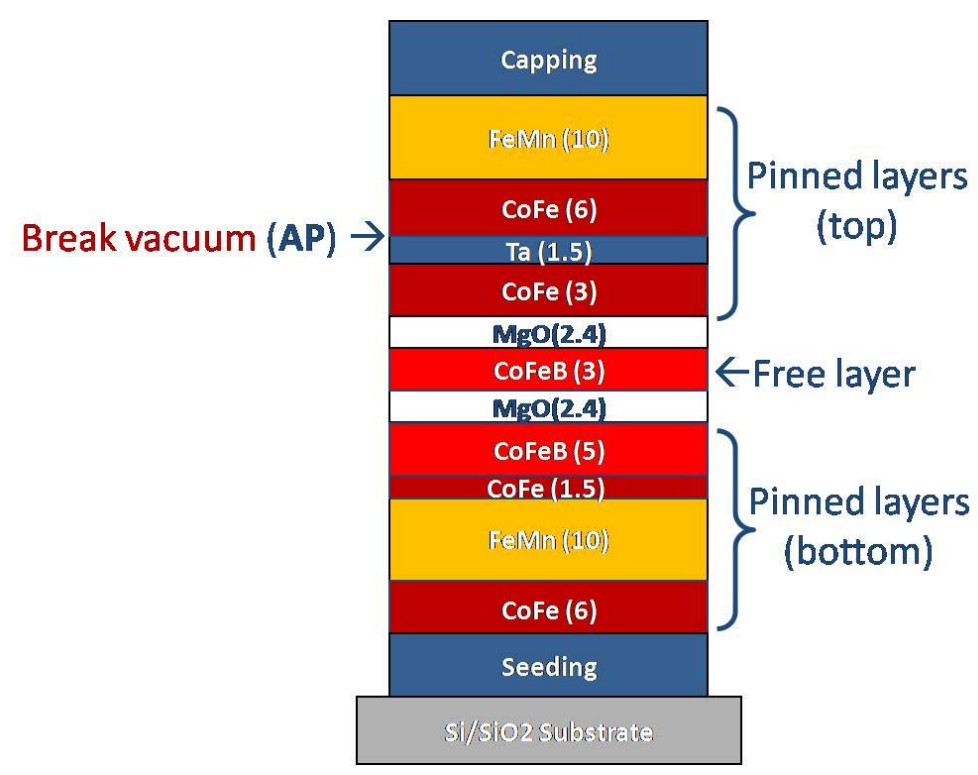

Figure 7.2 Material stack of double barrier MTJ with MgO barriers (units in nm). 


\subsection{Results and discussion}

\subsubsection{Single barrier MgO-MTJs}

\subsubsection{Barrier optimization for MgO-MTJs}

Figure 7.3 shows the $\mathrm{M}(\mathrm{H})$ loop of a typical single barrier $\mathrm{MgO}-\mathrm{MTJ}$ with structure as follows: Ta6/V2/Ta6/CoFe6/FeMn10/CoFe1.5/CoFeB5/MgO1.8/CoFeB5/Ta6 (nm). Clear magnetic loop separation was observed in the figure with minor loop centered around zero field and the $\mathrm{CoFe} 6 / \mathrm{FeMn} 10 / \mathrm{CoFe} 1.5 / \mathrm{CoFeB} 5 \mathrm{~EB}$ coupled pinned layer loop centered around 200 Oe. Figure 4 shows the corresponding TMR vs Field curve with data obtained from the CIPT simulation results. After annealing the sample at $350^{\circ} \mathrm{C}$ for $1 \mathrm{hr}$, a high TMR value of $70 \%$ was obtained from the sample deposited by our BTIBD system.

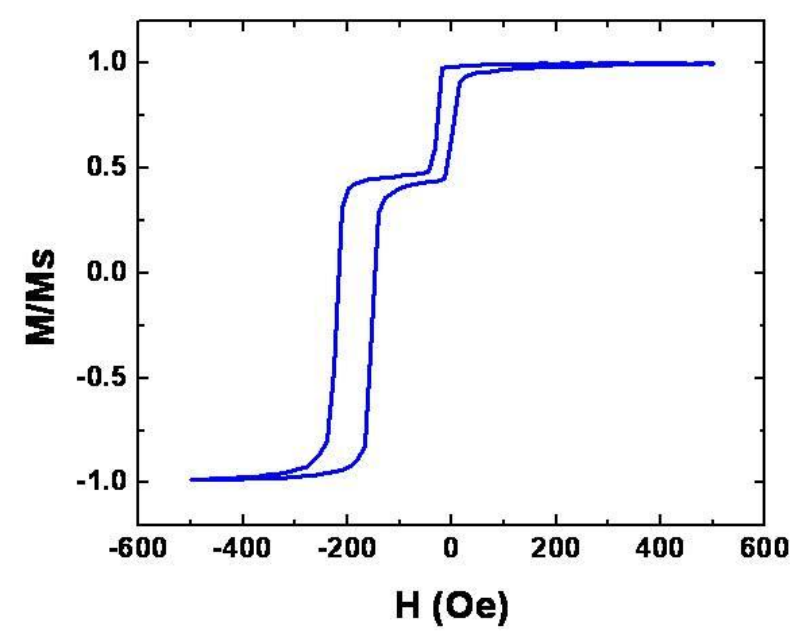

Figure 7.3 M(H) loop at RT of typical MTJ stack with MgO barrier. 


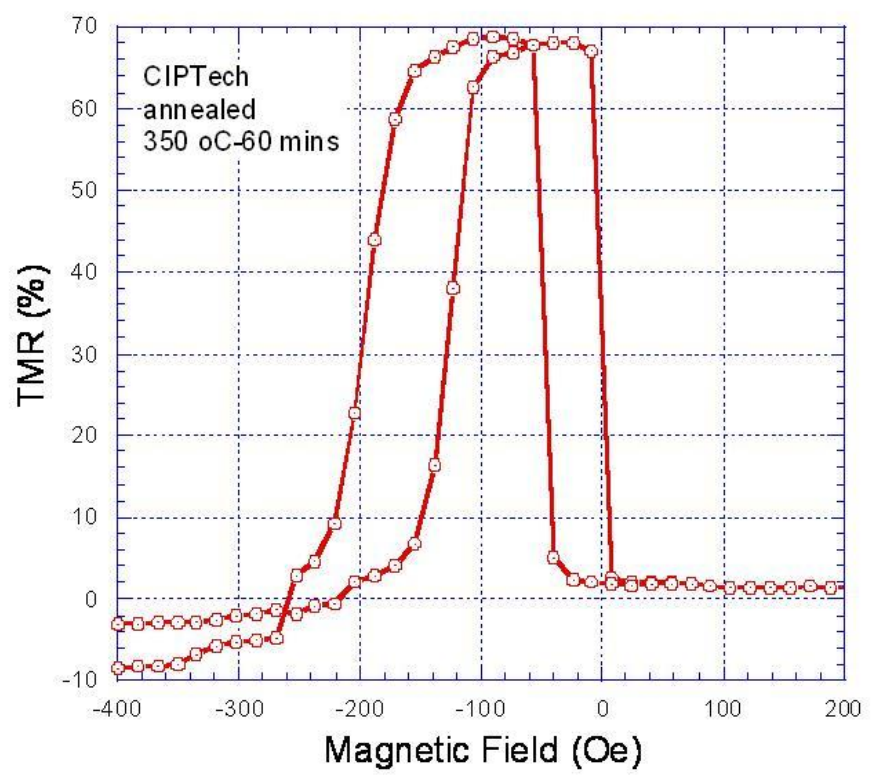

Figure 7.4 TMR vs magnetic field of a representative MgO-MTJ sample after 350C 1 hour annealing.

One of the first series of experiments was to study the barrier thickness dependence of the TMR based on the single barrier MgO-MTJs. The MTJ stack is as follows: Ta6/V2/Ta6/CoFe6/FeMn10/CoFe1.5/CoFeB5/MgO(1.8-3.2)/CoFeB5/Ta6 (nm). The thickness of the $\mathrm{MgO}$ barrier was varied from 1.8 to $3.2 \mathrm{~nm}$, each with $0.2 \mathrm{~nm}$ increase of the barrier thickness. The sample set was quickly field cooled at $280^{\circ} \mathrm{C}$ for $1 \mathrm{~min}$ to set the bottom EB pinning. The TMR was again measured by the CIPT method and the results were plotted below in Figure 7.5. The trend of barrier thickness dependence was very clear. When the barrier is relatively thin, the TMR decreases with smaller barrier thickness, which is probably due to the pinholes formed with thin barrier layer. With the increasing thickness of barrier, the pinholes were gradually filled and good insulating barrier was formed for better spin-polarized tunneling behavior. On the other hand, according to Simmons' expression [77], there exists an exponential dependence of the 
tunneling current with the barrier thickness inside the MTJ structure. So when the tunneling barrier thickness is large enough to form a good tunnel barrier, the corresponding tunneling current will decrease exponentially with an increase in barrier thickness, hence the TMR effect gets reduced correspondingly as well. The optimized barrier thickness was found to be around $2.4 \mathrm{~nm}$ to give the highest TMR with a $280^{\circ} \mathrm{C} 1$ min rapid thermal annealing condition.

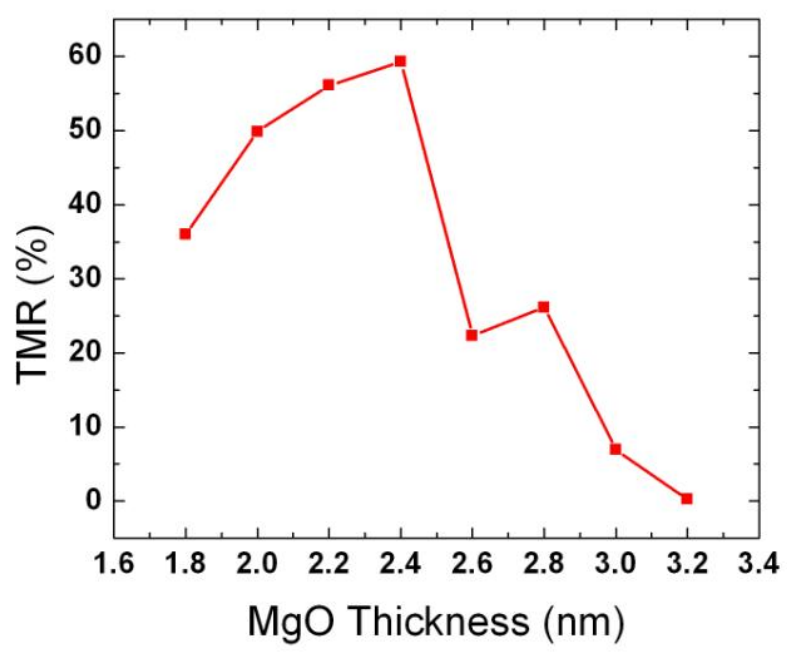

Figure 7.5 MgO barrier thickness dependence of TMR values. The MTJ sample set was quickly field cooled at $280^{\circ} \mathrm{C}$ for 1 min to set the bottom EB pinning.

After obtaining the optimized barrier thickness of $2.4 \mathrm{~nm}$ with the rapid thermal annealing condition at $280^{\circ} \mathrm{C}$ for $1 \mathrm{~min}$, the barrier layer oxidation conditions were also explored. The $\mathrm{MgO}$ barrier oxidation for all previous studies was carried out using 10 Torr oxygen for $10 \mathrm{~min}$ for each $1.2 \mathrm{~nm} \mathrm{Mg}$ metal layer, and an oxidization rate of $50 \mathrm{~s} / \AA$ was used for any $\mathrm{Mg}$ layer less than $1.2 \mathrm{~nm}$. To investigate the effects of different 
oxidation processes, a barrier layer was fixed at $2.4 \mathrm{~nm}$, and the two-step natural oxidation process was carried out for the barrier formation. For each $1.2 \mathrm{~nm} \mathrm{Mg}$ layers, the oxidation condition was summarized in the following Table 7-1.

Table 7-1 Effect of MgO barrier oxidation conditions on TMR value. Sample set was annealed at $350^{\circ} \mathrm{C}$ for $1 \mathrm{hr}$.

\begin{tabular}{|c|c|c|c|}
\hline Samples & Oxidation Pressure (Torr) & Oxidation Time (min) & TMR (\%) \\
\hline A & 10 & 10 & 34.15 \\
\hline B & 10 & 20 & 36.53 \\
\hline C & 100 & 10 & 51.59 \\
\hline D & 100 & 20 & 42.74 \\
\hline
\end{tabular}

From Table 7-1 we can see that for each $1.2 \mathrm{~nm} \mathrm{MgO}$ barrier formation, 10 Torr 10 min seems not strong enough to fully oxidize the $1.2 \mathrm{~nm} \mathrm{Mg}$ layer in each oxidation process. The TMR keeps increasing with longer annealing conditions among sample A, B and $\mathrm{C}$, with better quality $\mathrm{MgO}$ barrier formed with longer oxidation times. However, the TMR starts to drop with the oxidation condition of 100 Torr for 20 min for sample D. This is probably because the $\mathrm{MgO}$ barrier was over oxidized so that the surface of the underlying $\mathrm{CoFeB}$ got oxidized. Such an oxidized layer at the interface, even as thin as a few monolayers, is detrimental to the spin-polarized tunneling through the barrier because it causes significant random electron scattering and depolarize the electrons, and hence reduces the TMR performance. 


\subsubsection{Annealing optimization for MgO-MTJs}

As we know, the annealing treatment is an essential step to obtain high TMR value. This is because high quality crystalline $\mathrm{MgO}$ sharing a good interface with crystallized bcc-CoFeB is the key to obtain highly spin-polarized tunneling through an MgO-MTJ structure [23], The only effective way to obtain crystallized $\mathrm{MgO}$ and $\mathrm{CoFeB}$ around the interface is through the annealing treatment. Optimization of the annealing condition is known to be necessary to obtain high TMR values. On one hand, if the annealing treatment is too weak, the $\mathrm{MgO}$ barrier won't be fully crystallized to achieve high spinpolarized tunneling performance. On the other hand, if the annealing condition is too strong, in practical MTJs with FeMn, IrMn or PtMn antiferromagnetic layers, the active Mn atoms will obtain enough energy to diffuse across the whole MTJ stack, and cause problems including the failure of separate magnetic switching, or degraded TMR performance due to the contamination of the $\mathrm{MgO}$ barrier with Mn diffusion[27] .

To study the annealing effect on the MTJ properties, first two sets of identical samples were deposited, each containing four samples with the following structures: Ta6/V2/Ta6/CoFe6/FeMn10/CoFe1.5/CoFeB5/MgO(1.8-2.4)/CoFeB5/Ta6 (nm), and the only difference among these samples was the thickness of the $\mathrm{MgO}$ barrier. These two sets of identical samples were annealed using two different annealing conditions to examine the annealing impact. One set was annealed at $280^{\circ} \mathrm{C}$ for $1 \mathrm{~min}$ to quickly set the EB pinning, while the other set was annealed at $350^{\circ} \mathrm{C}$ for $1 \mathrm{hr}$ to study the effect of long annealing. The TMR values for samples with different barrier thicknesses and two distinct annealing conditions were shown in the following Figure 7.6. 


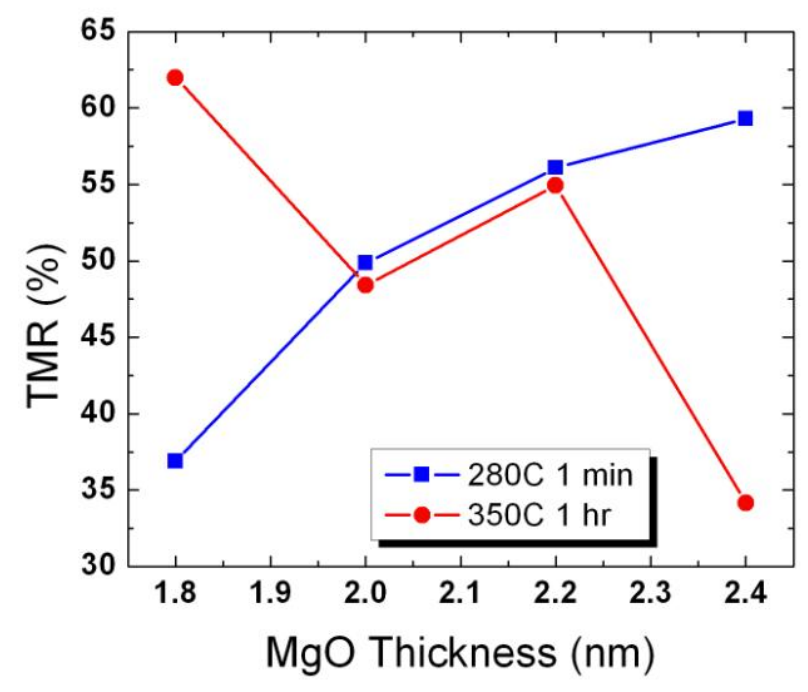

Figure 7.6 Annealing effect of MgO-MTJs with different barrier thickness.

Two different trends of TMR dependence on the $\mathrm{MgO}$ barrier thickness could be observed from Figure 7.6. For samples with quick field cooling at $280^{\circ} \mathrm{C}$, higher TMR was obtained with larger $\mathrm{MgO}$ barrier thickness. This was explained in the previous section 7.3.1.1. With an optimized barrier thickness around $2.4 \mathrm{~nm}$, this set of samples was deposited with relatively thin barriers. The thinner the barrier becomes, more pinholes and other defects would lead to low TMR performance with the same annealing condition. However, with the stronger annealing condition at $350^{\circ} \mathrm{C}$ for $1 \mathrm{hr}$, MTJs with larger barrier thickness actually showed lower TMR. This opposite trend indicates that somehow after a $350^{\circ} \mathrm{C} 1 \mathrm{hr}$ anneal, the optimized $\mathrm{MgO}$ barrier thickness seems to be shifted to the lower end. This is probably because such strong annealing conditions help to fully crystallize both the $\mathrm{MgO}$ barrier and the $\mathrm{CoFeB}$ electrodes as well. Better spinpolarized tunneling efficiency could be obtained with a highly crystallized $\mathrm{CoFeB} / \mathrm{MgO} / \mathrm{CoFeB}$ structure, compared to the situation with $\mathrm{CoFeB}$ only partially 
oxidized with a few monolayers at the interfaces after quick field cooling. This explains why the MTJ sample with a $1.8 \mathrm{~nm} \mathrm{MgO}$ barrier shows much higher TMR after a $350^{\circ} \mathrm{C}$ $1 \mathrm{hr}$ anneal compared to the sample with a $280^{\circ} \mathrm{C} 1$ min quick field cooling. Since the optimized barrier thickness shifts to lower values using MTJs with a strong anneal. This explains why with larger barrier thicknesses, the TMR starts to drop among samples with $350^{\circ} \mathrm{C} 1 \mathrm{hr}$ annealing. The conclusion we can draw here is that the annealing step is essential to obtain high TMR for MTJs, and the optimized barrier thickness would actually change depending on the different annealing conditions.

To further optimize the TMR performance, another important parameter of MgOMTJs, the thickness of the $\mathrm{CoFeB}$ layers, was also varied. One of the motivations for optimizing the thickness of $\mathrm{CoFeB}$ layers, both as the free layer and as a part of the pinned reference layer, is to minimize the Néel coupling field when using the MTJ as part of the hard disk drive (HDD) recording head, so that the sensitivity of the read head could be improved. One way to reduce the Néel coupling field is to use thinner CoFeB layers as indicated by equation 6.1. However, if the CoFeB FM layers are too thin, they won't be able to detect enough spin polarized current, consequently the TMR value will be reduced. Here the thicknesses of the two $\mathrm{CoFeB}$ layers inside the MTJ stacks was varied and different annealing conditions were applied to investigate the impact of both annealing and $\mathrm{CoFeB}$ thickness on TMR performance. Three identical sets of MgO-MTJ samples were deposited. Within each set of five samples, the only difference is thickness of the two $\mathrm{CoFeB}$ layers above and below the $\mathrm{MgO}$ barrier layer. The MTJ stack is as follows: $\quad$ Ta6/V2/Ta6/CoFe6/FeMn10/CoFe1.5/CoFeB(X)/MgO(2.4)/CoFeB(X)/Ta6 $(\mathrm{nm})$. Here the thickness of the two CoFeB layers $\mathrm{X}$ was varied from $4.5 \mathrm{~nm}$ to $2.5 \mathrm{~nm}$ 
with a step of $0.5 \mathrm{~nm}$. Three different annealing conditions were carried out, with annealing temperature at 200,250 , and $350^{\circ} \mathrm{C}$ while keeping the same annealing time at 1 hr. The results of TMR values of samples with different CoFeB thickness and three distinct annealing conditions were shown in the following Figure 7.7.

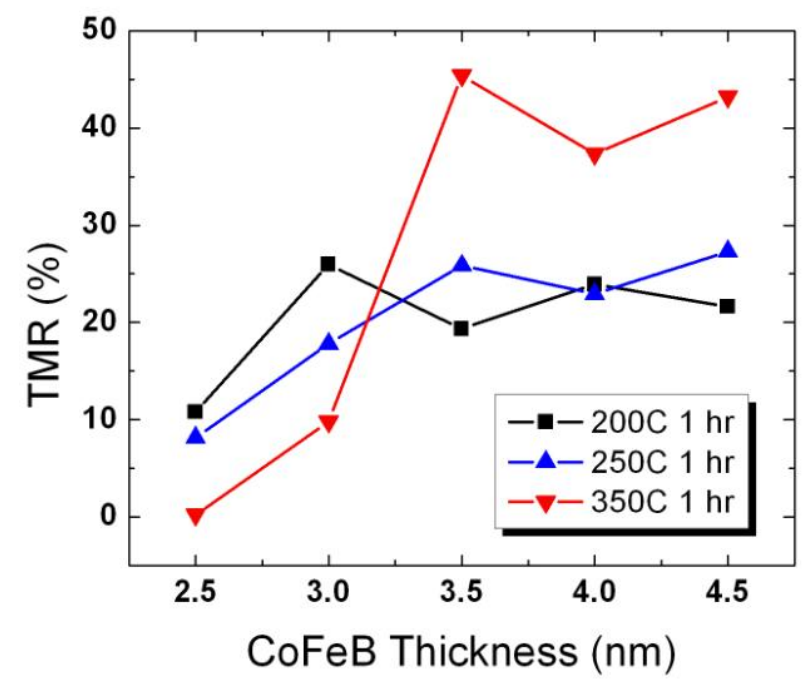

Figure 7.7 Annealing effect of MgO-MTJs with different CoFeB layer thickness.

Figure 7.7 shows that there is a critical thickness of CoFeB layers around $3.5 \mathrm{~nm}$, below which thinner CoFeB results lower TMR values. This observation is obvious for the sample sets annealed at $250^{\circ} \mathrm{C}$ and $350^{\circ} \mathrm{C}$. For the sample set annealed at $200^{\circ} \mathrm{C}$, the TMR value with $\mathrm{CoFeB}$ thickness at $3.0 \mathrm{~nm}$ shows an abnormally high value, which is not yet understood. But the lowest TMR value at $2.5 \mathrm{~nm}$ for all three sets with different annealing conditions confirms that if the $\mathrm{CoFeB}$ layers below or above the $\mathrm{MgO}$ barrier are too thin, they would not be able to provide or detect enough spin-polarized current to achieve large TMR values. Above the critical thickness of $3.5 \mathrm{~nm}$, the TMR values seem 
to be insensitive to the $\mathrm{CoFeB}$ thickness with the same annealing condition. Therefore, for best TMR performance with minimum Néel coupling interference, $3.5 \mathrm{~nm}$ would be the optimized CoFeB thickness for MTJs for HDD reader sensor applications. To further investigate the annealing effect on TMR, the samples with extreme CoFeB thickness were examined. On one hand, for the samples with the thinnest $\mathrm{CoFeB}(2.5 \mathrm{~nm})$ layers, higher annealing temperature results in lower TMR values. This is probably because it is relatively easy for thin $\mathrm{CoFeB}$ layers to crystallize and higher annealing temperature would actually cause more diffusion of Mn across the whole film stack, which degrades TMR performance. On the other hand, for samples with thick $\mathrm{CoFeB}(4.5 \mathrm{~nm})$ layers, higher annealing temperature turned out to be necessary to form higher quality $\mathrm{CoFeB}$ and $\mathrm{MgO}$ barriers as well as the $\mathrm{CoFeB} / \mathrm{MgO} / \mathrm{CoFeB}$ interfaces, which is essential for high TMR performance. Also, thicker $\mathrm{CoFeB}$ layers would be more effective in reducing or even blocking the diffusion of Mn atoms during the annealing process. This explains why above the critical thickness of $3.5 \mathrm{~nm}$, higher TMR values were generally obtained for all three annealing conditions.

\subsubsection{Double barrier MgO-MTJs}

The motivation for making double barrier MTJs (DBMTJs) is either to enhance the TMR ratio performance with two exchange bias (EB) pinned layers parallel to each other, or to reduce the critical switching current in the STT switch mode $[78,79]$ when the two pinned layers are configured to be anti-parallel to each other. Our preliminary results 
showed that by applying different fabrication procedures, DBMTJs with the two EB pinned layers in either parallel or antiparallel configurations could be fabricated.

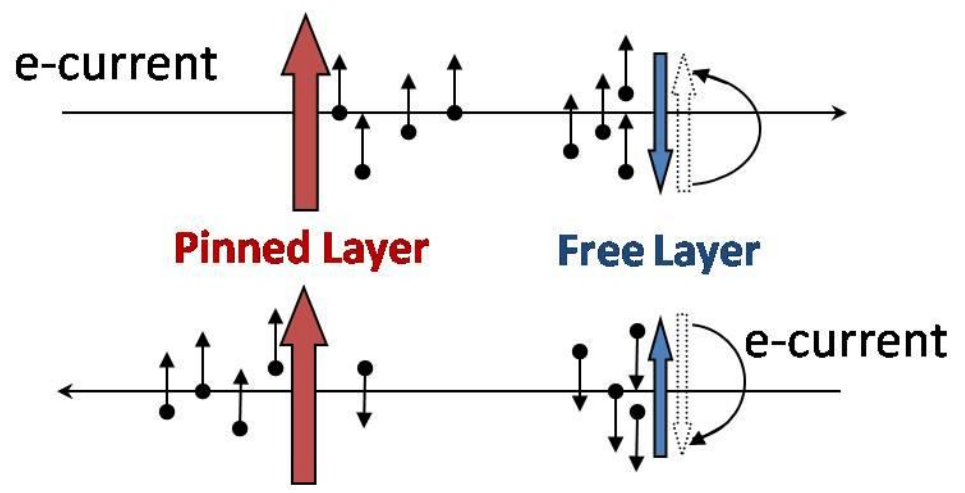

Figure 7.8 Illustration of spin torque transfer (STT) switching mechanism for single barrier MTJ with two different initial states, with the pinned layer and free layer either parallel or antiparallel to each other before the STT switching.

To understand the benefit of DBMTJs, the mechanisms of Spin Torque Switching (STT) in both single barrier MTJ and DBMTJ are compared as follows. Figure 7.8 shows the Spin Torque Switching mechanism of a single barrier MTJ. If the initial state of the two magnetizations of the pinned land free layers is antiparallel, we inject electron from pinned layer to the free layer. The pinned layer acts like the spin polarizer and only electrons with up spins get passed through the pinned layer. When such up spin electrons enter the free layer with opposite magnetic alignment, the free layer will receive spin torque and eventually flip its magnetization, as shown in the top part of Figure 7.9. On the other hand, if the initial state of the two magnetizations is parallel, we need to inject electrons from the free layer to pinned layer. In this case, the pinned layer acts as spin filter. The spin-up electrons will pass through the pinned layer freely while the spin-down electrons get reflected from the pinned layer. The reflected spin down electrons start to 
accumulate near the free layer surface. Eventually, the free layer receives enough spin torque from those spin down electrons and its magnetization gets flipped, as shown in the bottom part of Figure 7.8 .

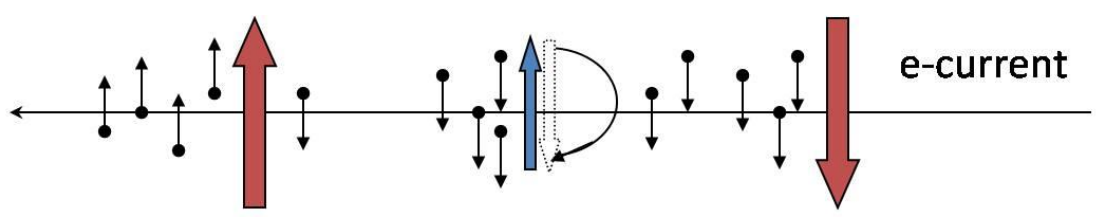

Pinned Layer $\quad$ Free Layer Pinned Layer

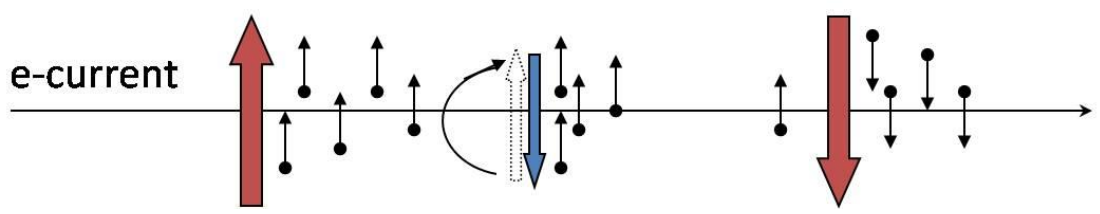

Figure 7.9 Illustration of STT switching mechanism for double barrier MTJ (two pinned layers are configured antiparallel to each other) with two different initial states.

In the case of DBMTJ, there are two configurations with the two pinned layer either parallel or antiparallel to each other. For STT-RAM applications, the antiparallel configuration is the preferred state with the advantage of reducing the critical switching current density. As shown above in Figure 7.9, when the free layer moment is aligned up, we inject electrons from right to left. In this case, the right pinned layer acts like the spin polarizer, providing electrons with down spins that could exert torque on the free layer with up magnetization. While the left pinned layer acts like the spin filter, allowing spin up electrons to pass through and reflecting electrons with down spins. So the free layer receives spin torques from the spin down electrons at both sides. That is why the critical switching current density could be lowered. The disadvantage of this configuration is that there always exists an antiparallel configuration of the free layer and one of the pinned 
layers, so very little tunneling magnetoresistance (TMR) value would be expected unless we change the tunnel barrier layer thickness to create asymmetric tunneling on the two sides of the free layer. That is why there is always the need to balance of the reduced TMR value with a lowered critical switching current. The priority of engineering depends on which parameter is more important in actual applications.
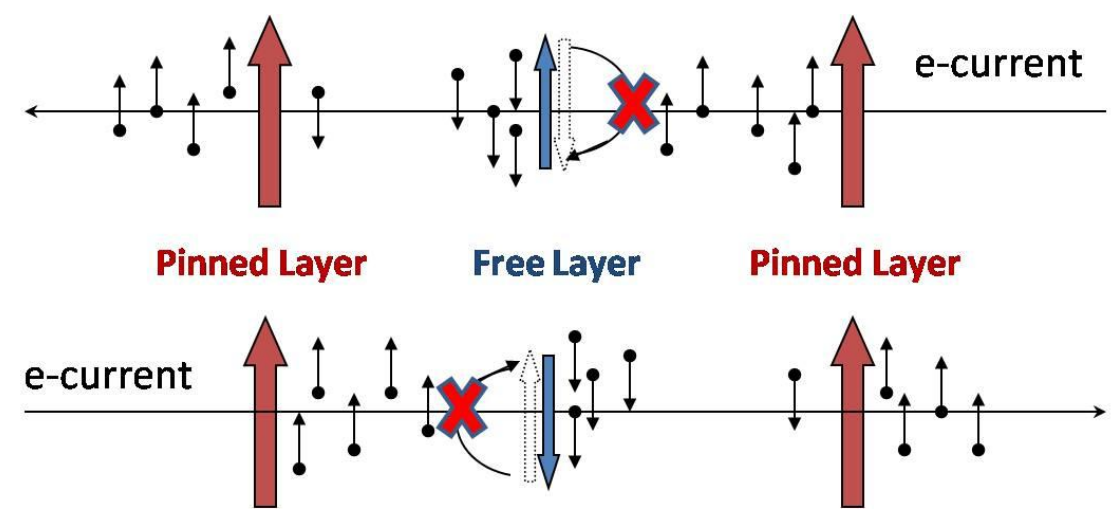

Figure 7.10 Illustration of STT switching mechanism for double barrier MTJ (two pinned layers are configured parallel to each other) with two different initial states.

In another configuration when the two pinned layers are parallel to each other, as shown in Figure 7.10, in either case when the free layer magnetization is up or down, the spins of electrons accumulated on each side of the free layer are in opposite directions, so no effective spin torque will be exerted on the free layer to flip its magnetizations. That is why this configuration is not suited for STT-RAM application which requires low switching current density. However, this configuration has its own advantage when used as the read head sensor in HDD applications. In this case external fields from the magnetic bits in the hard disk cause the free layer moment to rotate. Thus during the read operation, a sense current will be passed through the MTJ structure to read out the 
resistance high or low state. Due to the thermal fluctuations, even a small sensing current could occasionally rotate free layer magnetization due to the STT switching effect, which will cause error during the read operation [80]. DBMTJ with two parallel pinned layers will have minimized STT switching effects due to the two opposite contributions with current flowing in either direction, and will also have enhanced TMR as we just explained. That is why DBMTJs with parallel pinned layers are well suited for the read head sensor in HDD applications.

As we explained in the experimental part in section 7.2.2, the basic structure of a DBMTJ deposited with two pinned layer either parallel or antiparallel is as follows: Substrate/Ta6/V2/Ta6/CoFe6/FeMn10/CoFe1.5/CoFeB5/MgO1.2/MgO1.2/CoFeB3/Mg O1.2/MgO1.2/CoFe3/Ta1.5/CoFe6/FeMn10/Ta10 (all units in nm).

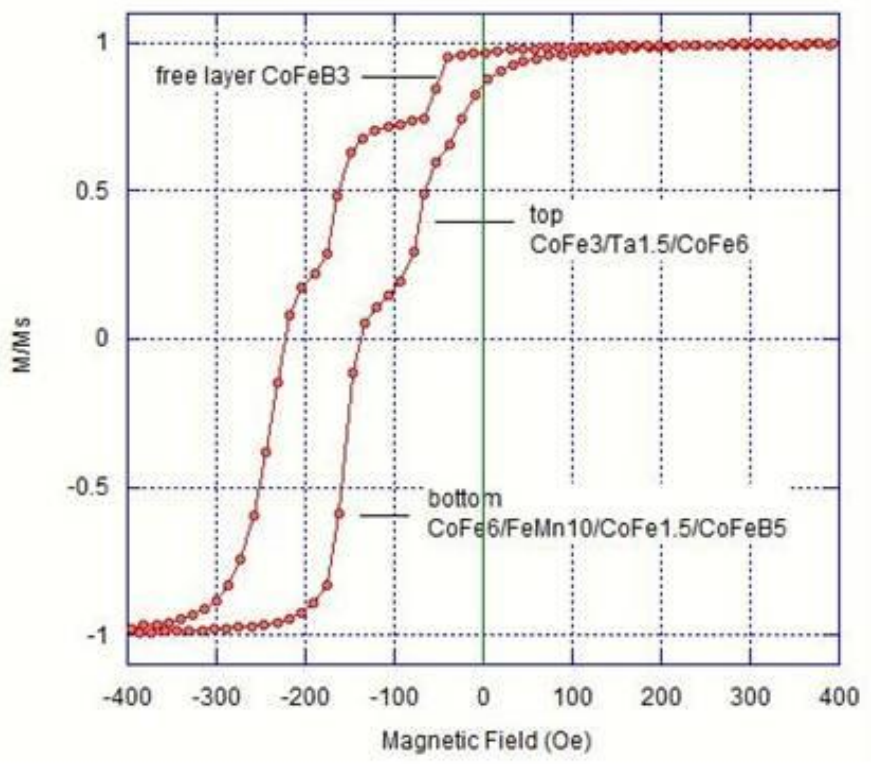

Figure 7.11 M(H) loop of DBMTJ with two parallel pinned layers. 
Figure 7.11 shows the $\mathrm{M}(\mathrm{H})$ loop of a MgO-DBMTJ with two parallel pinned layers. When the magnetic field sweeps from positive to negative, the free layer switches first with the free loop center shifted a bit toward the negative field. This is mainly due to the Néel coupling with both pinned layers sitting above or below, both of which are negatively exchange biased. We can see from Figure 7.11 that the top $\mathrm{CoFe} / \mathrm{Ta} / \mathrm{CoFe}$ composite layers still couples together even with a $1.5 \mathrm{~nm}$ Ta insertion layer. The top EB pinned layer $\mathrm{CoFe} / \mathrm{Ta} / \mathrm{CoFe} / \mathrm{FeMn}$ has a relatively smaller $\mathrm{EB}$ field compared to the bottom EB pinned layer, based on the two clearly separated EB loops from Figure 7.11.

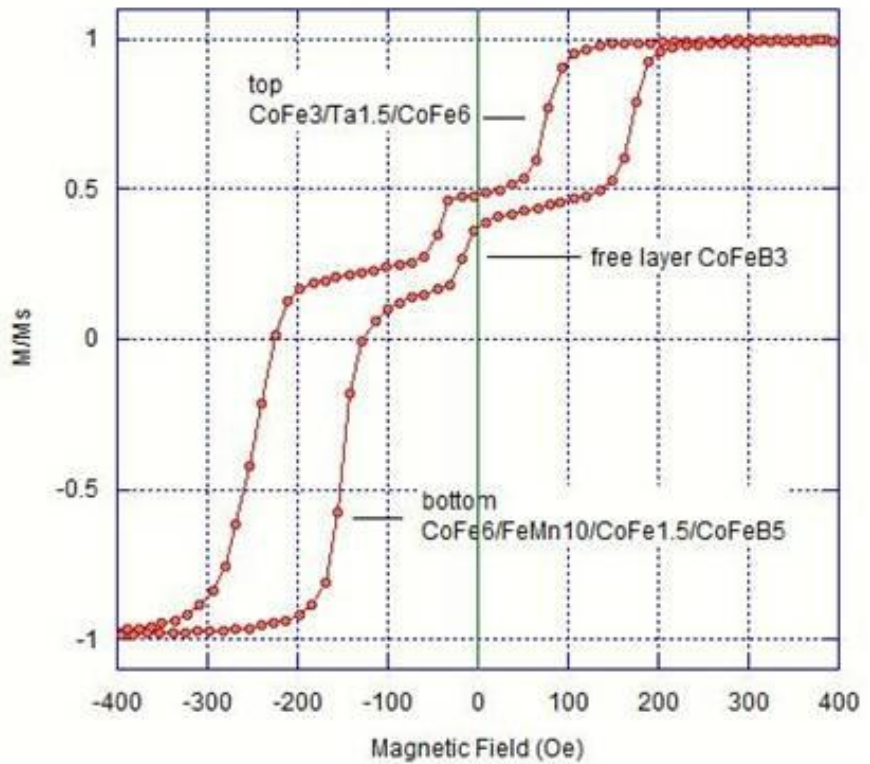

Figure 7.12 M(H) loop of DBMTJ with two antiparallel pinned layers.

An $\mathrm{M}(\mathrm{H})$ loop of an MgO-DBMTJ with two antiparallel pinned layer is shown in Figure 7.12. The top pinned layer switches first due to the positively EB coupling set during the deposition process. Then the free layer switches around zero field again with a 
slight loop center shift towards the negative field. This is because the thicker bottom pinned layer provides stronger Néel coupling to the free layer compared to the one induced with the top pinned layers. The bottom pinned layer switches at negative field around 200 Oe, a little bit larger than the top EB field, which is consistent with the DBMTJ with parallel pinned layers. Due to the complexity of the BDMTJ structure, it is difficult to measure its TMR directly with the traditional CIPT method, which is equipped with an algorithm for single barrier MTJ only. After the successful deposition of DBMTJs with pinned layers either parallel or antiparallel, our next step is to send the blanket films to our collaborators at NIST for photolithographic patterning before carrying out further electrical and magnetic characterizations.

\subsection{Summary}

In this chapter, the deposition of MgO-MTJs with the BTIBD system was benchmarked. Optimization work was carried out by varying material stack parameters, processing conditions as well as the post-deposition annealing conditions. By varying the $\mathrm{MgO}$ barrier layer thickness, the highest TMR value was achieved with an $\mathrm{MgO}$ thickness around $2.4 \mathrm{~nm}$ under rapid thermal annealing conditions at $280^{\circ} \mathrm{C}$ for $1 \mathrm{~min}$. By further improving the oxidation conditions during the barrier formation step, a higher TMR value was obtained. Also, an annealing treatment was found to be an essential step in getting a high quality crystallized $\mathrm{MgO}$ barrier and $\mathrm{CoFeB}$ FM electrodes for good spin-polarized tunneling. The optimum barrier thickness was found to be dependent on annealing conditions, and smaller optimized $\mathrm{MgO}$ thickness was found with stronger 
annealing conditions. The CoFeB FM electrode thickness was optimized to minimize the Néel coupling effect while maintaining high TMR, which is suitable for HDD read head sensor applications. Lastly, some preliminary results on DBMTJs show that DBMTJs with two pinned layer either parallel or antiparallel could be successfully fabricated, and both DBMTJs with either configuration are suited for various technological applications. 


\section{Exploration of $\tau-\mathrm{MnAl}$ as electrodes for MTJs}

\subsection{Introduction}

The ferromagnetic $\tau$ phase of the MnAl binary alloy was first discovered by Kono in 1958[81], followed by extensive investigation of its structure and magnetic properties mainly on the bulk alloy samples instead of thin films. It is well known that the $\tau$ phase of $\mathrm{MnAl}$ with $\mathrm{Mn}$ atomic concentrations around $50 \sim 60 \%$ is metastable and possesses excellent hard magnetic properties even without traditional ferromagnetic elements like Co, Fe and Ni. According to the initial study from Kono, this $\tau$ phase, which is the only magnetic phase of $\mathrm{MnAl}$, has a tetragonal structure with lattice constant $\mathrm{a}=2.79 \AA$ and $\mathrm{c}$ $=3.57 \AA$ and lattice positions $(0,0,0)$ and $(1 / 2,1 / 2,1 / 2)$ with a preference for $\mathrm{Mn}$ atoms in one of these positions and $\mathrm{Al}$ atoms in the other. Figure 8.1 shows the structure and partial phase diagram of MnAl taken from Ref. [82]
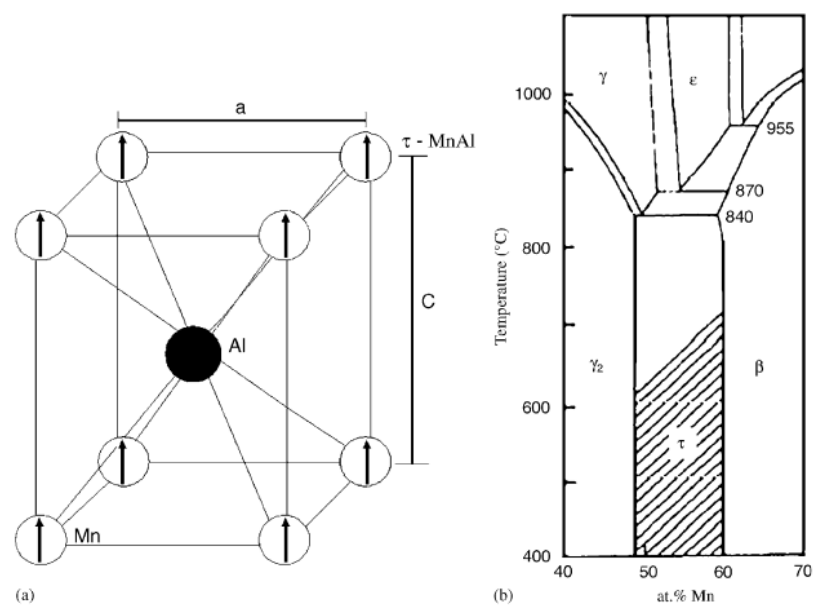

Figure 8.1 (a) Schematic of $\tau$-MnAl structure, the magnetic moments of the Mn atoms are parallel to the c-axis of the MnAl, (b) part of the Mn-Al phase diagram showing the $\gamma, \gamma_{2}, \tau$, $\beta$ and $\varepsilon$-phases. 
The occurrence of ferromagnetism originates from the distorted distance between neighboring Mn atom pairs with a value of $2.79 \AA$. This value is larger than the interatomic distance of $2.57 \AA$, where exchange coupling between $3 \mathrm{~d}$-electrons of adjacent Mn atoms changes from antiferromagnetic to ferromagnetic based on the BetheSlater-curve[81]. The ferromagnetic $\tau-\mathrm{MnAl}$ has remarkable magnetic properties such as a high $\mathrm{K}_{\mathrm{u}}$ about $10^{7} \mathrm{ergs} / \mathrm{cm}^{3}$ and a saturation magnetization around $490 \mathrm{emu} / \mathrm{cc}[83]$. All these values fall into the right range of parameters desired for improving the properties of the spin torque transfer (STT) switching behavior of magnetic tunnel junctions (MTJs) for next generation STT-RAM applications. Also, the advantage of perpendicular anisotropy compared with in-plane magnetization lies in the fact that there is an extra $2 \pi M_{S}$ term that the spin torque needs to overcome in the in-plane case, while it doesn't contribute to the thermal stability [37]. Therefore, less current would be required to switch the free layer with perpendicular anisotropy. Various thin film deposition techniques were applied for the fabrication of ferromagnetic $\tau$-MnAl, including magnetron sputtering[48, 84], molecular beam epitaxy (MBE)[85] and pulsed laser deposition (PLD)[86]. The successful growth of $\tau-\mathrm{MnAl}$ with perpendicular magnetic anisotropy (PMA) was realized by MBE and the proper choice of substrate template, while most films made by sputtering were polycrystalline and lacked the correct crystal orientation. However, sputtering is a much more desired method to fabricate such magnetic thin films for industrial applications, so here I explored the ferromagnetic $\tau$ phase MnAl thin film synthesis by using Reactive Bias Target Ion Beam Deposition (RBTIBD). 
It is well known that the $\tau$ phase in the MnAl binary alloy system is metastable and cannot be obtained without special heat treatment such as quenching and annealing[48]. As shown in Figure 8.2, the widely accepted mechanism states that during the annealing treatment, the high temperature nonmagnetic $\varepsilon$ phase (hcp) transforms into a nonmagnetic $\varepsilon$ ' phase (orthorhombic) by an ordering reaction, then transforms into a metastable ferromagnetic $\tau$ phase (fct) by a martensitic transition [87]. Sputtering is one of the superquenching processes, but still the as-deposited $\mathrm{MnAl}$ thin films show almost no magnetic behavior if not deposited using elevated temperature. For films deposited at ambient temperature, the ferromagnetic $\tau$ phase can only be obtained with proper postdeposition annealing treatment. That is why the annealing treatment becomes so important in the formation of the $\tau$ phase.

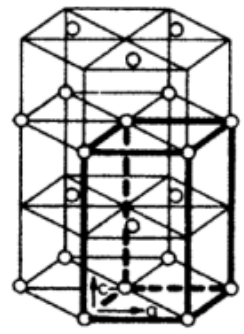

A3 ( $(\varepsilon)$

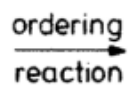

reaction

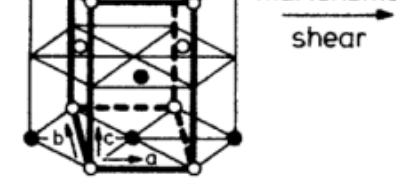

B19 ( $\left.\varepsilon^{\prime}\right)$

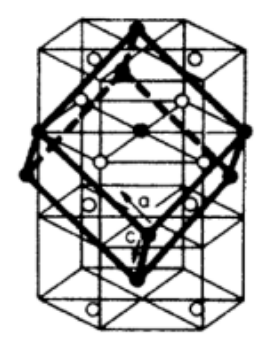

$\mathrm{L1}_{0}(\tau)$

Figure 8.2 Proposed phase transformation sequence and the lattice relationship during the formation of $\tau$ phase MnAl according to Ref. [87].

In this chapter, I systematically studied the growth conditions and the postdeposition annealing effects on the structural and magnetic behaviors of $\tau$-MnAl thin films, as well as the dependence of film thickness and the effects of various interfaces on 
the structural and magnetic properties of $\tau-\mathrm{MnAl}$, with the purpose of optimizing the properties of $\tau-\mathrm{MnAl}$ for its future incorporation into MTJs for potential STT-RAM applications.

\subsection{Experiments}

As I mentioned before when I introduced RBTIBD in chapter 2, during the cosputtering process of more than one target, the relative amounts of the different target materials deposited onto the substrate are controlled by varying the target pulse frequency and the positive pulse period. For all the MnAl thin film samples discussed in this chapter, I set the pulse frequency of the $\mathrm{Mn}$ and $\mathrm{Al}$ targets to be the same i.e. $50 \mathrm{kHz}$, which leads to a total pulse period of $20 \mu \mathrm{s}$. The parameters that I varied were the positive pulse widths applied to the $\mathrm{Mn}$ and $\mathrm{Al}$ targets, which were controlled separately by two different pulse generators. When I chose $4 \mu \mathrm{s}$ as the input for the positive pulse width for the Mn target, the negative pulse width became $16 \mu \mathrm{s}$, which was the actual Mn target sputtering duration. Similarly, for $3 \mu$ s as the positive pulse width for Al target, the actual negative biased sputtering duration was $17 \mu$ s for $\mathrm{Al}$ metal sputtering. By varying the relative positive pulse width on these two targets, the atomic compositions of $\mathrm{Mn}$ and Al could be controlled very precisely.

Here I describe briefly the typical procedures of making $\tau$-MnAl thin films for our study. First, the ultrasonically cleaned sample substrates were mounted on the wafer carrier using silver paint before I fixed the wafer carrier onto the sample stage in the load lock chamber. The load lock was then pumped down by the mechanical pump to 90 
mTorr before the isolation gate valve between load lock chamber and the high vacuum processing chamber was opened. After the sample stage was pushed into the processing chamber I waited until the whole system vacuum went down below $6 \times 10^{-8}$ Torr. I started the purge of the cathode with $10 \mathrm{sccm}$ argon for $30 \mathrm{~min}$ followed by another 30 min-degas of the cathode filament. Then I turned on the ion source by applying voltages to both cathode and anode guns. The stage shutter was then opened to expose the sample substrates to the plasma for 5 min gentle ion milling to further clean the sample surfaces before the actual film deposition. Next, the stage shutter was closed and the voltage pulses for the $\mathrm{Mn}$ and $\mathrm{Al}$ targets were turned on. I used a negative $600 \mathrm{~V}$ and positive 20 $\mathrm{V}$ as the sputtering voltages to pre-clean the targets before film deposition. After a 10 min pre-clean, the bias voltages were turned off while the ion source was kept in the idle state for 10 min to cool down the whole system. Even though the targets are water cooled by running water through the backing plates, a significant amount of heat could still be generated and would heat up the target surfaces and also would heat the neighboring region in the deposition chamber if the targets are sputtered for a long period of time. This could cause the system parameters like gun voltage, current, bias current, etc. to be unstable and ultimately would affect the deposition rate which I assume constant throughout the whole sputtering process. Such a pre-cleaning and pre-sputtering protocol as well as the idle time steps was strictly followed for all film depositions to ensure the consistency of film qualities.

For most MnAl thin films made in this study, an approximate $6 \mathrm{~nm}$ Ta capping layer was usually deposited to prevent the surface oxidation of MnAl, especially during the post-deposition annealing process that is an essential step to create the ferromagnetic $\tau$ 
phase of MnAl. The following Table 8-1 shows the typical process conditions for MnAl and Ta films deposited with the RBTIBD:

Table 8-1 Typical process conditions for MnAl and Ta film deposition by RBTIBD

\begin{tabular}{|c|c|c|}
\hline Growth Parameters / Film ID & $\mathrm{MnAl}$ & $\mathrm{Ta}$ \\
\hline Negative Bias Voltage (V) & 600 & 300 \\
\hline Negative Bias Current (mA) & 103 & 84 \\
\hline Positive Bias Voltage (V) & 27.4 & 27.2 \\
\hline Positive Bias Current (A) & 1.01 & 0.65 \\
\hline Cathode Ar Flow (sccm) & 10 & 10 \\
\hline Cathode Voltage (V) & 7.2 & 3.1 \\
\hline Cathode Current (A) & 5.04 & 7.06 \\
\hline Anode Ar Flow (sccm) & 50 & 70 \\
\hline Anode Voltage (V) & 32.1 & 37.0 \\
\hline Anode Current (A) & 2.51 & 6.55 \\
\hline Target \#1 Frequency $(\mathrm{kHz})$ & $(\mathrm{Mn}) 50$ & (Ta)71.43 \\
\hline Target \#1 Positive Pulse Width ( $\mu$ s) & 4 & 3 \\
\hline Target \#2 Frequency $(\mathrm{kHz})$ & (Al) 50 & N/A \\
\hline Target \#2 Positive Pulse Width $(\mu s)$ & 3 & N/A \\
\hline Substrate Temperature $\left({ }^{\circ} \mathrm{C}\right)$ & Ambient & Ambient \\
\hline Process Pressure (mTorr) & 0.75 & 0.98 \\
\hline
\end{tabular}

Several different annealing methods were used to investigate the annealing effects on the structural and magnetic behaviors of the $\tau$-MnAl thin films in order to optimize the film properties. In chapter 3 I introduced the two annealing furnaces that were used for the heat treatment. One is a vacuum furnace with a base pressure around $5 \times 10^{-6}$ Torr and an optional fixed magnetic field around $400 \mathrm{Oe}$, and the other is equipped with a forming 
gas (Ar and $\mathrm{H}_{2}$ mixture with 5 at. $\% \mathrm{H}_{2}$ ) and strong adjustable external field up to $5 \mathrm{kOe}$. Another annealing option was to use the heating lamp in the RBTIBD for in situ annealing. This option was very useful when I explored the properties of $\tau$-MnAl with different interfaces. In particular, when I tried to make MnAl thin films without any capping, the in situ annealing became essential to obtain the $\tau$-MnAl without taking the samples out of the deposition chamber and then transferring to another annealing furnace. In this case the surface oxidation during annealing wasn't much of a concern, thanks to the high vacuum in the RBTIBD, around $5 \times 10^{-8}$ Torr.

The magnetic hysteresis loops were measured using a Lakeshore vibrating sample magnetometer (VSM) which has a rotating sample holder so both in-plane and out-ofplane loops could be measured. Also the attached VSM option of a Physical Properties Measurement System (PPMS) by Quantum Design was used to measure the in-plane loops to double check the measurement accuracies. The results showed that the data obtained from the Lakeshore VSM and PPMS were very consistent and agreed with each other very well. X-ray Diffraction (XRD) measurements were carried out using the Rigaku Smart Lab system and the detailed data analysis work was finished based on collaboration with colleagues at College of William \& Mary.

\subsection{Results and discussions}

\subsubsection{Selection of substrate templates}

The proper choice of substrate as the growth template is crucial in determining the structural and magnetic properties of thin film materials. For example, $\mathrm{MgO}(001)$ is 
widely used as the preferred substrate for growing $\mathrm{L} 1_{0} \mathrm{FePt}$ thin films with perpendicular anisotropy (PMA). The $\mathrm{L1}_{0} \mathrm{FePt}$ films have a very similar structure to the $\tau$-MnAl. The lattice constant of $\mathrm{MgO}$ is $4.21 \AA$, and the a axis of FePt is around $3.86 \AA$, so the $9.07 \%$ lattice mismatch helped to expand the a axis of FePt and shrink the $\mathrm{c}$ axis and this favors the $\mathrm{L} 1_{0}$ ordering even at a relatively low growth temperature[88]. In the case of MBE growth of $\tau$-MnAl, $\{100\} \mathrm{GaAs}$ was chosen as the template due to only a $2 \%$ lattice mismatch between the a axis of $\mathrm{MnAl}$ and the $\mathrm{a} / 2$ of GaAs[85].

The motivation for the $\tau-\mathrm{MnAl}$ thin film exploration is to utilize its unique magnetic properties as electrodes for MTJs for a possible STT-RAM application. In particular, due to the relatively low magnetic anisotropy and coercivity of $\tau$-MnAl compared with other perpendicular electrode candidates like FePt, it is best suited as the free layer of MTJs. The goal would be to achieve low current STT switching. As I mentioned in the previous chapters, crystallized $\mathrm{MgO}(001)$ is so far the best tunnel barrier material with high spin filtering efficiency and a record high tunneling magnetoresistance (TMR), so $\mathrm{MgO}(001)$ as the substrate template for studying $\tau-\mathrm{MnAl}$ thin film properties becomes a very natural choice. Nevertheless, as the initial step of this investigation, ten different types of substrates available in our lab were picked and mounted on the sample stage under the same deposition conditions to check the effect of different substrates on the magnetic properties of the MnAl thin films.

As I pointed out in the introductory part of this chapter, the formation of $\tau$-MnAl is very sensitive to the relative composition of $\mathrm{Mn}$ and $\mathrm{Al}$, thus by choosing the proper positive pulse width for $\mathrm{Mn}(3 \mu \mathrm{s})$ and $\mathrm{Al}(4 \mu \mathrm{s})$, I were able to obtain the largest saturation magnetization, i.e. the largest amount of $\tau$ phase MnAl with ambient 
temperature growth. The details of the composition dependence of $\tau$-MnAl properties will be discussed in the following section. For this set of samples labeled L100409, ambient temperature growth was used, which means the heating lamp under the sample stage of RBTIBD was not turned on during the deposition process. The deposition time for the $\mathrm{MnAl}$ film layer of the sample set was set for $1 \mathrm{hr}$ with nominal thickness around $120 \mathrm{~nm}$, and $6 \mathrm{~nm}$ Ta was deposited following the MnAl deposition as the capping layer. All of the as-deposited films showed very weak magnetic signals beyond the sensitivity of our VSM system, so annealing was necessary to facilitate the above mentioned phase transformation from $\varepsilon \rightarrow \varepsilon^{\prime} \rightarrow \tau$. As shown on Table 8-2, the saturation magnetization of all ten samples with different substrates were measured after these samples were annealed using our vacuum furnace at $400^{\circ} \mathrm{C}$ for $30 \mathrm{~min}$ without applying external magnetic field.

The results turned out to be very encouraging. The $\mathrm{MnAl}$ thin film on the $\mathrm{MgO}(001)$ substrate showed a significantly higher value of the saturation magnetization than any other types of substrate used in this study. This confirms our motivation for using $\tau$-MnAl as the electrode for MTJs with $\mathrm{MgO}(001)$ tunnel barriers and the $\tau$ - $\mathrm{MnAl} / \mathrm{MgO}(001)$ interface, since $\mathrm{MgO}(001)$ seems to be a good choice of seed layer for the successful growth of $\tau$-MnAl. Based on this preliminary result, I concluded that an $\mathrm{MgO}(001)$ template produced the largest amount of $\tau$ phase $\mathrm{MnAl}$ with the highest saturation magnetization.

Table 8-2 Saturation magnetization of MnAl (1hr deposition) on different substrates. Here MgO(BTIBD) refers to $6 \AA$ A of Mg deposited by BTIBD on a thermally oxidized Si substrate, followed by natual oxidation when exposed in air before being put in the RBTIBD as a growth template; $\mathrm{SiO}_{2}$ 
refers to a thermally oxidized $\mathrm{Si}$ substrate with a $\sim 3000 \AA \mathrm{SiO}_{2}$ oxidation layer. All other substrates are commercially available products from various suppliers.

\begin{tabular}{|c|c|c|c|c|c|c|c|c|c|c|}
\hline Substrate type & $\begin{array}{l}\mathrm{Al}_{2} \mathrm{O}_{3} \\
(0001)\end{array}$ & $\begin{array}{l}\mathrm{SrTiO}_{3} \\
(100)\end{array}$ & $\begin{array}{l}\mathrm{TiO}_{2} \\
(001)\end{array}$ & $\begin{array}{l}\mathrm{TiO}_{2} \\
(100)\end{array}$ & $\begin{array}{l}\mathrm{TiO}_{2} \\
(110)\end{array}$ & $\begin{array}{l}\mathrm{TiO}_{2} \\
(011)\end{array}$ & $\begin{array}{l}\mathrm{MgO} \\
(111)\end{array}$ & $\begin{array}{l}\mathrm{MgO} \\
(001)\end{array}$ & $\begin{array}{l}\mathrm{MgO} \\
\text { (BTIBD) }\end{array}$ & $\mathrm{SiO}_{2}$ \\
\hline $\mathrm{M}_{\mathrm{S}}(\mu \mathrm{emu})$ & 55 & 215 & 20 & 20 & 20 & 20 & 170 & 900 & 40 & 90 \\
\hline Crystal Structure & hexagonal & cubic & \multicolumn{4}{|c|}{ Tetragonal } & \multicolumn{2}{|l|}{ cubic } & unknown & amorphous \\
\hline $\begin{array}{l}\text { Lattice } \\
\text { constant }(\AA)\end{array}$ & $\begin{array}{l}\mathrm{a}=4.79 \\
\mathrm{c}=12.99\end{array}$ & $a=3.91$ & \multicolumn{4}{|c|}{$\begin{array}{l}a=4.95 \\
c=2.96\end{array}$} & \multicolumn{3}{|c|}{$\mathrm{a}=4.21$} & N/A \\
\hline
\end{tabular}

The substrate denoted as "SiO2" on Table 8-2 was a thermally oxidized Si substrate with a SiO2 thickness about $3000 \AA$. The saturation magnetization $\sim 30$ emu/cc could be calculated from the total moment of $90 \mu \mathrm{emu}$, sample size $5 \times 5 \mathrm{~mm}$ and thickness about $120 \mathrm{~nm}$, which is only a fraction of value of bulk $\tau-\mathrm{MnAl}(\sim 490 \mathrm{emu} / \mathrm{cc}[83])$. It means that only a very small percentage (less than $10 \%$ ) of MnAl was $\tau$ phase even after the annealing treatment. As we know from its properties in the bulk, $\tau$-MnAl has a tetragonal structure with $\mathrm{a}=2.79 \AA$ and $\mathrm{c}=3.57 \AA$, and this causes a huge lattice mismatch with the a-axis of $\mathrm{TiO}_{2}$ which has the value of $4.95 \AA$, so $\mathrm{MnAl}$ films grown on $\mathrm{TiO}_{2}$ templates with different orientations showed a very weak magnetic signal, even smaller than that of the $\mathrm{MnAl}$ on the amorphous $\mathrm{SiO}_{2}$ substrate, which implies that such templates favored other non-magnetic phases of MnAl. Similar analysis could be applied to the case of the $\mathrm{Al}_{2} \mathrm{O}_{3}$ substrate with a relative much larger a-axis value of $4.79 \AA$.

Figure 8.3 shows the $\mathrm{MvsH}$ hysteresis loops measured both in-plane with magnetic field parallel to the film surfaces and out-of-plane with the field perpendicular to the film plane for four different substrates. Figure 8.3 (a) and (b) show the $\mathrm{MnAl}$ films on $\mathrm{SiO}_{2}$ and the naturally oxidized $\mathrm{MgO}(\mathrm{BTIBD})$ film on the $\mathrm{SiO}_{2}$ substrate, respectively. In 
Figure 8.3 (b), the Mg layer was first deposited by our BTIBD system and the film was exposed to air before being put into the RBTIDB system. This was how the naturally oxidized $\mathrm{MgO}$ served as the $\mathrm{MnAl}$ growth template. The crystal structure of the $\mathrm{MgO}(\mathrm{BTIBD})$ layer was not certain but most likely was amorphous. Due to the possible inferior surface smoothness and uniformity compared with the thermally oxidized $\mathrm{Si}$ substrate, the $\mathrm{MnAl}$ grown on $\mathrm{MgO}(\mathrm{BTIBD})$ might have more defects and dislocations which caused the even smaller amounts of magnetic $\tau$-MnAl compared to that on $\mathrm{SiO}_{2}$. On the other hand, as shown in Figure 8.3 (c), MnAl grown on $\mathrm{SrTiO}_{3}$ (001) substrates showed much higher magnetic signal than on most other substrates. This is probably due to the relatively smaller lattice mismatch between $\mathrm{a}=3.91 \AA$ of $\mathrm{SrTiO}_{3}(001)$ and $\mathrm{c}=$ $3.57 \AA$ of $\tau$-MnAl. This also explains why the hysteresis loop showed sharp in-plane loop switching, indicating an in-plane easy axis was observed compared to the more linear hard-axis response of the out-of-plane hysteresis loop. In the tetragonal structure of $\tau$ $\mathrm{MnAl}$, the magnetization of the $\mathrm{Mn}$ atoms is along the c-axis, making the c-axis the magnetic easy axis. So in this case the c-axis of $\tau$ - $\mathrm{MnAl}$ on $\mathrm{SrTiO}_{3}$ lies in plane due to the close lattice match with the $\mathrm{SrTiO}_{3}(001)$ substrate. 

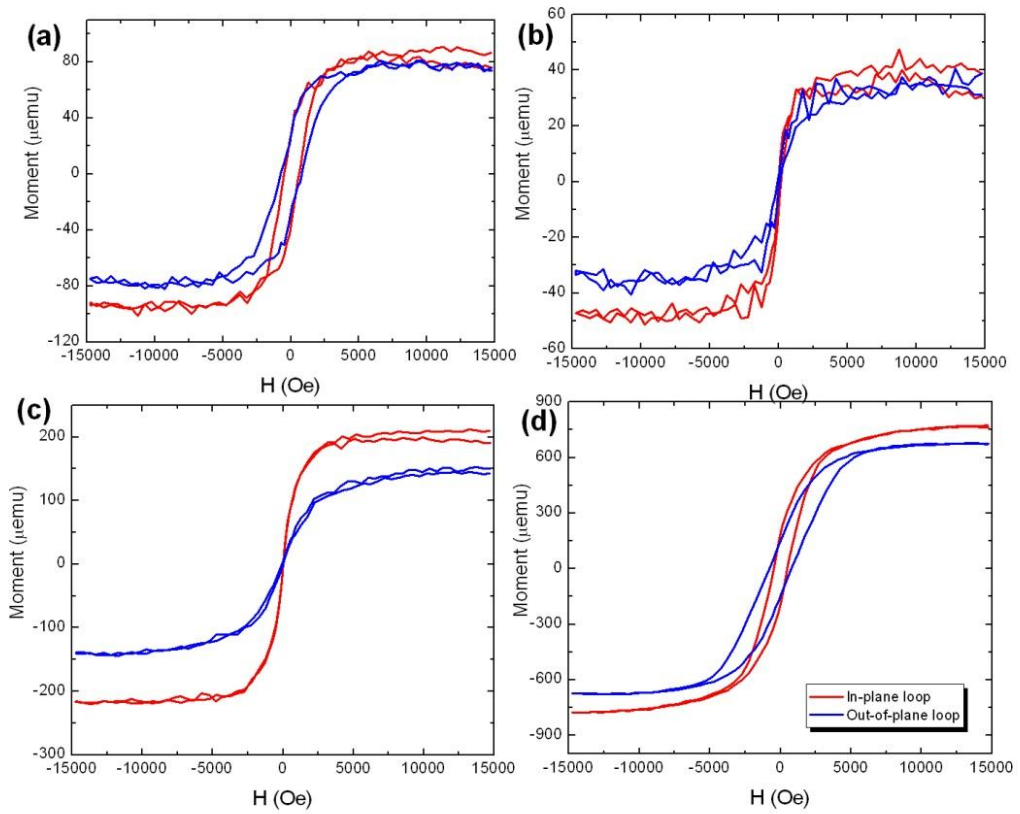

Figure 8.3 MvsH loops for $1 \mathrm{hr}$ deposition of MnAl thin films on different substrates: (a) $\mathrm{SiO} 2$ (thermally oxidized Si wafer) (b) $\mathrm{MgO}(\mathrm{BTIBD})$ (c) $\mathrm{SrTiO3}$ (100) (d) $\mathrm{MgO}(001)$

Finally, in Figure 8.3 (d), the $\mathrm{MnAl}$ grown on the $\mathrm{MgO}(001)$ substrate showed the highest saturation magnetization among all substrates, with a rough estimate of the saturation magnetization of $\sim 300 \mathrm{emu} / \mathrm{cc}$, over $60 \%$ of the standard bulk value. This means $60 \%$ of the $\mathrm{MnAl}$ film grown on the $\mathrm{MgO}(001)$ substrate after annealing was the ferromagnetic $\tau$-phase. Also both in-plane and out-of-plane coercivity of $\tau$-MnAl on $\mathrm{MgO}(001)$ were much larger than those grown on other substrates, indicating that a much more ordered $\tau-\mathrm{MnAl}$ crystal structure was formed in this case. I believe that the main contribution to this successful growth of $\tau-\mathrm{MnAl}$ was the close lattice match between the $\mathrm{MgO}(001)$ substrate and the $\tau$-MnAl. A more detailed analysis will be discussed in the 
following section, mainly based on the results from X-Ray Diffraction (XRD) measurements.

\subsubsection{Epitaxial growth of $\tau$-MnAl on $\mathrm{MgO}(001)$}

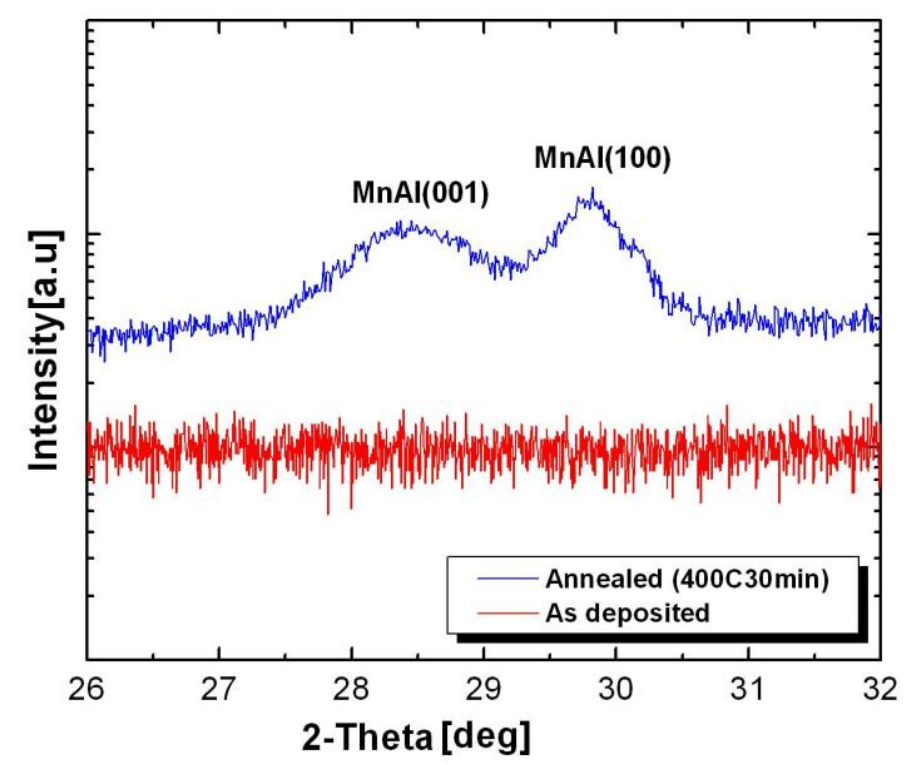

Figure 8.4 XRD 2-Theta/Omega scan of both as-deposited MnAl sample L100409 on MgO(001) substrate and the same sample after annealing $\left(400^{\circ} \mathrm{C} 30 \mathrm{~min}\right)$

Figure 8.4 shows the XRD 2-Theta/Omega scan of the MnAl sample L100409 on a $\mathrm{MgO}(001)$ substrate both as-deposited and after annealing at $400 \mathrm{C}$ for $30 \mathrm{~min}$. The saturation magnetization value of the annealed $\tau-\mathrm{MnAl}$ thin film sample is $\sim 300 \mathrm{emu} / \mathrm{cc}$ (over $60 \%$ of the bulk $\tau$-MnAl value). The $\tau-\mathrm{MnAl}(001)$ and (100) peaks that are clearly observed confirm the existence of the $\tau$-MnAl phase, both with in-plane (100) and out-ofplane (001) orientations. The co-existence of both orientations was also confirmed by the 
in-plane and out-of-plane hysteresis loops, which had similar loop shape and coercivity with similar magnitude. The calculated lattice constants from the (100) $(2$-Theta $=$ $\left.29.7636^{\circ}\right)$ and $(001)\left(2-\right.$ Theta $\left.=28.3962^{\circ}\right)$ peaks are $\mathrm{a}=3.00 \AA$ and $\mathrm{c}=3.14 \AA$ respectively. These values are slightly different from the bulk values of $\tau-\mathrm{MnAl}(\mathrm{a}=2.79$ $\AA$ and $\mathrm{c}=3.57 \AA$ ). However, if I assume the epitaxial growth relationship between $\tau$ $\mathrm{MnAl}$ and the $\mathrm{MgO}(001)$ template with cubic structure, the lattice matching between $\mathrm{Mn}$ (or $\mathrm{Al}$ ) atoms to the neighboring $\mathrm{Mg}$ (or Oxygen) atoms would lead to the conclusion that the lattice distance between $\mathrm{Mn}$ or $\mathrm{Al}$ atoms should be close to $\mathrm{a}_{\mathrm{MgO}} / \sqrt{2}=4.21 / \sqrt{2}=2.98$ $\AA$, and there would be a $45^{\circ}$ offset between the crystal orientations of $\mathrm{MgO}$ and $\tau$-MnAl. The lattice matching was confirmed by the measured values of $\tau-\operatorname{MnAl}(\mathrm{a}=3.00 \AA$ and $\mathrm{c}$ $=3.14 \AA$ ). The best analogy reflecting the $45^{\circ}$ offset the epitaxial growth relationship is presented in Figure 8.5, which shows the well studied $\mathrm{Fe}(001) / \mathrm{MgO}(001)$ system within the magnetic tunnel junction system. The lattice constant of the bcc structured $\mathrm{Fe}(001)$ is $2.87 \AA$, and it is very close to that of $\mathrm{MgO}(001)$, with $\mathrm{a}_{\mathrm{MgO}} / \sqrt{2}=2.98 \AA$.

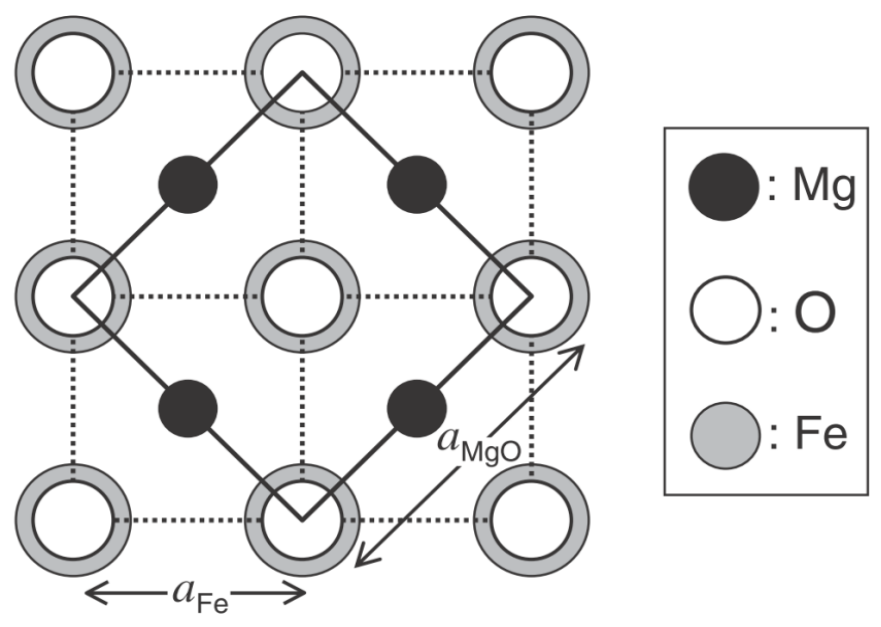

Figure 8.5 The epitaxial growth of $\mathrm{Fe}(001)$ on $\mathrm{MgO}(001)$ template due to close the lattice matching. It also shows clearly the $45^{\circ}$ offset of the growth orientation between the two structures, from Ref. [27] 
To further confirm the assumption of epitaxial growth of $\tau$-MnAl on $\mathrm{MgO}(001)$, I rotated the $\chi$ angle of the sample for $45^{\circ}$, so that the (100) orientation of $\tau$-MnAl became the (110) direction. I located the (110) peak using a 2-Theta/Omega scan with the peak 2Theta value around $42.9608^{\circ}$. This corresponds to a lattice constant value $\mathrm{a}_{\mathrm{MnAl}}=2.975$ $\AA$, very close to direct value of $3.00 \AA$ from the $\operatorname{MnAl}(100)$ peak. I fixed the 2 theta and omega angle of the (110) peak and did the $360^{\circ} \Phi$ scan to get the four (110) peaks due to the four-fold symmetry of the tetragonal structure of $\tau$-MnAl. Similarly, the same $\chi$ angle rotation of $45^{\circ}$ also transferred the $\mathrm{MgO}(200)$ peak to (220). By locating the $\operatorname{MgO}(220)$ peak and performing a $360^{\circ} \Phi$ scan around the fixed (220) direction, four (220) peaks were obtained due to the four-fold symmetry of the $\mathrm{MgO}$ cubic structure. As shown in Figure 8.6, the four peaks of MnAl (110) maintains the rotation offset of $\Delta \Phi=45^{\circ}$ compared to the $\mathrm{MgO}(220)$ peaks. This clearly indicates the epitaxial growth relationship between the $\tau$ - $\mathrm{MnAl}$ film and the $\mathrm{MgO}(001)$ substrate template with matching lattice constants and $45^{\circ}$ rotation offset between the two crystal structures, similar to the $\mathrm{Fe} / \mathrm{MgO}(001)$ growth relationship illustrated above in Figure 8.5.

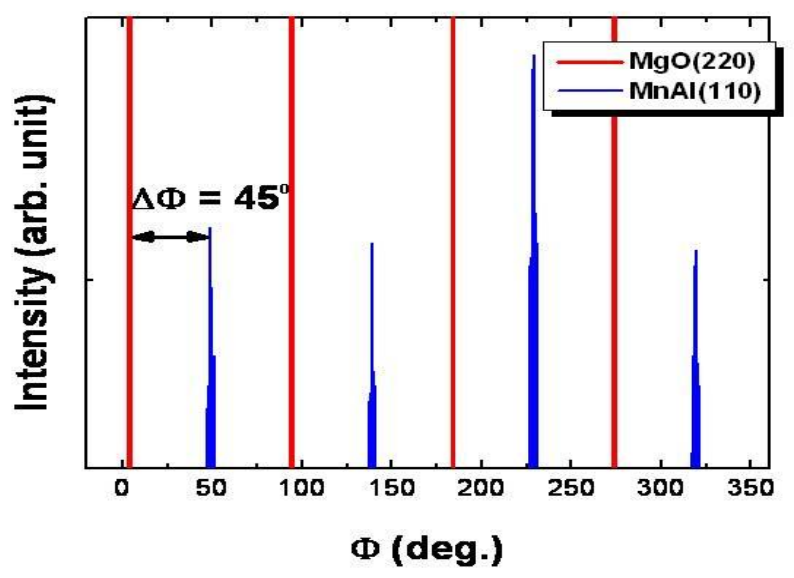

Figure 8.6 360 ${ }^{\circ} \Phi$ scan of $\mathrm{MgO}(220)$ and $\mathrm{MnAl}(110)$ peaks measured by XRD, showing the $45^{\circ}$ offset 


\subsubsection{Composition dependence of $\tau$-MnAl properties}

As we know, apart from the special heat treatment the processing window in terms of $\mathrm{Mn}$ and $\mathrm{Al}$ composition ratio is very small to obtain the $\tau$-MnAl. As we can see from the phase diagram of $\mathrm{MnAl}$ alloy in Figure 8.7, the $\tau-\mathrm{MnAl}$ in the bulk form only exists when the atomic concentration of $\mathrm{Mn}$ is within the range of 48 to $60 \%$ [82]. For thin films, as reported by Huang et al. [84], the ferromagnetic $\tau$ phase appeared at the composition range between 40 to 60 at. $\%$, and the maximum $\mathrm{M}_{\mathrm{S}} \sim 400 \mathrm{emu} / \mathrm{cc}$ was obtained for the $\mathrm{Mn}_{50} \mathrm{Al}_{50}$ films.

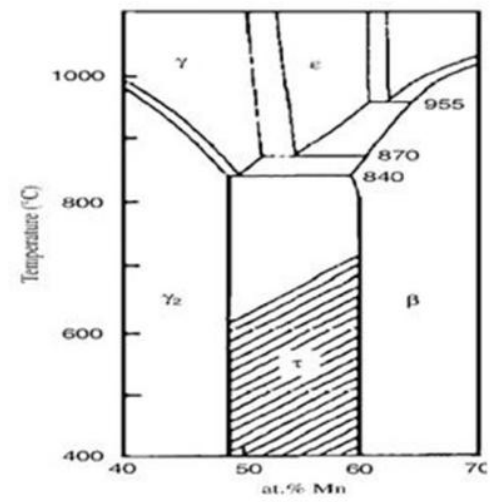

(a)

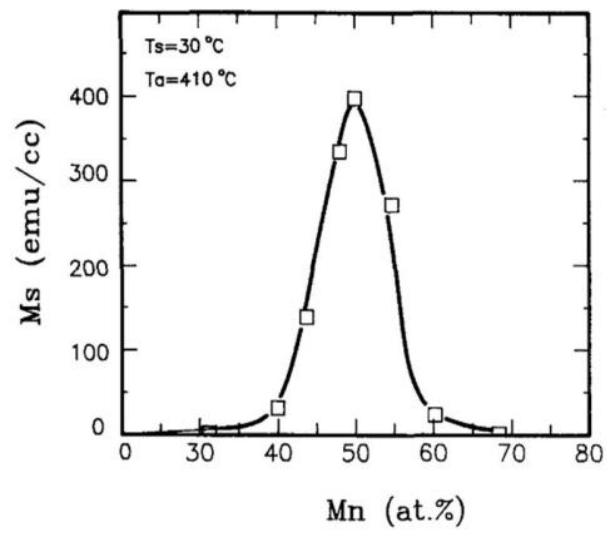

(b)

Figure 8.7 (a) Taken from Ref. [82], the phase diagram of MnAl alloy in the bulk form. The $\tau$ phase MnAl can only exist with Mn atomic concentration from 50 to $60 \%$. (b) Taken from Ref. [84], the saturation magnetization dependence on the Mn atomic concentration, with highest $M_{S} \sim 400 \mathrm{emu} / \mathrm{cc}$ obtained with a Mn concentration around $50 \%$.

To study the compositional dependence of the $\tau$-MnAl thin films prepared by our RBTIBD system, I deposited a series of MnAl samples by controlling the relative pulse 
width on the Mn and $\mathrm{Al}$ targets during the co-sputtering of the two targets. Specifically, I set the frequency of the DC pulses of both the $\mathrm{Mn}$ and $\mathrm{Al}$ targets to be $50 \mathrm{kHz}$, which corresponds to a total pulse period of $20 \mu \mathrm{s}$. I fixed the positive pulse width on the $\mathrm{Al}$ target to be $3 \mu \mathrm{s}$, and varied the positive width on the Mn target from 1 to $5 \mu \mathrm{s}$. As mentioned in the equipment introduction part, the smaller positive width means larger negative pulse duration and longer effective sputtering time, because only at negative pulse periods will the target actually be sputtered. Here with $1 \mu$ s positive pulse width, the Mn concentration measured within the MnAl alloy is the highest value of $54.89 \%$; while with $5 \mu$ s positive pulse width, a low boundary value of $49 \%$ was obtained for the Mn concentration. All of the films were deposited at ambient temperature for $60 \mathrm{~min}$ and vacuum annealed at $400^{\circ} \mathrm{C}$ for $30 \mathrm{~min}$ before the magnetic measurements. The detailed $\mathrm{M}_{\mathrm{S}}$ dependence on $\mathrm{Mn}$ concentration from our experimental data is plotted in Figure 8.8. It shows that the $\tau$-MnAl could be formed with Mn concentrations from 49 to $55 \%$ by the RBTIBD technique and post-deposition annealing treatment. The highest $\mathrm{M}_{\mathrm{S}} \sim 300$ emu/cc was obtained with a Mn concentration at 50.6\%. Our results are consistent with previous studies using other thin film growth techniques. 


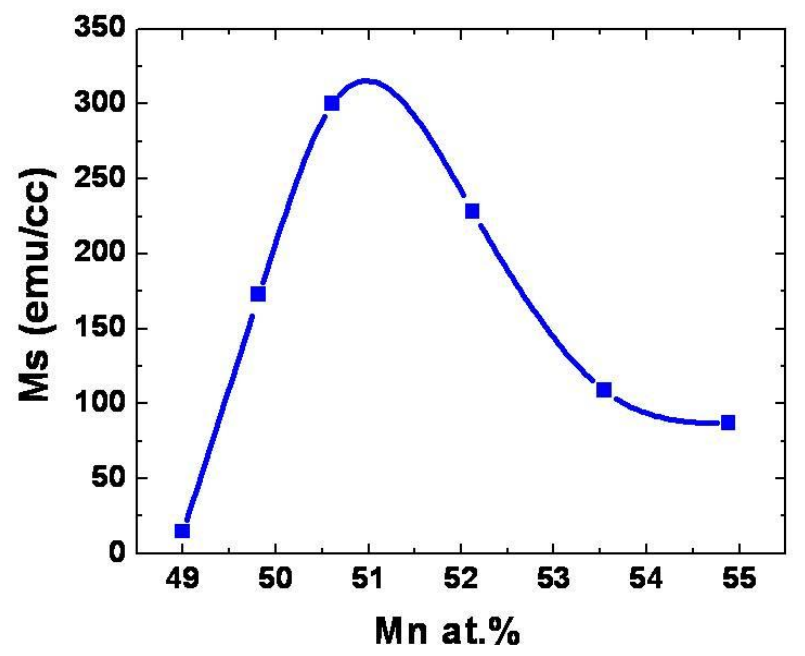

Figure 8.8 The saturation magnetization dependence on Mn atomic concentration from the MnAl samples prepared by RBTIBD. Samples were annealed at $400^{\circ} \mathrm{C}$ for 30 min before the measurement.

\subsubsection{Correlation between chemical ordering and saturation magnetization}

Figure 8.9 shows the normalized hysteresis loops of a representative sample (sample ID: L092409) with both in-plane and out-of-plane measurement. This sample was deposited at ambient temperature on an $\mathrm{MgO}(001)$ substrate for $20 \mathrm{~min}$, with a nominal thickness around $40 \mathrm{~nm}$ measured by $\mathrm{X}$-ray reflectivity. The optimized positive pulse width on the Mn target $(4 \mu \mathrm{s})$ was chosen to achieve the highest $\mathrm{M}_{\mathrm{S}}$ with a Mn atomic concentration $\sim 50.6 \%$ as shown in Figure 8.8. Post-deposition vacuum annealing at $400^{\circ} \mathrm{C}$ for 30 min was carried out before the characterization. 


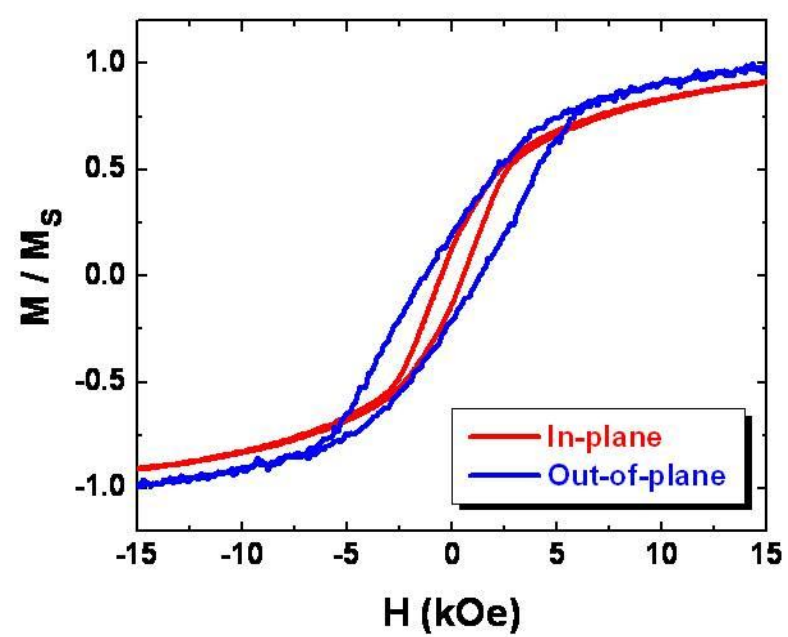

Figure 8.9 Hysteresis loops of sample L092409 with both in-plane and out-of-plane measurement.

The in-plane and out-of-plane coercivities extracted from the above hysteresis loops are 520 and 1300 Oe, respectively. Based on previous studies, we know that the moments of the $\mathrm{Mn}$ atoms responsible for the magnetic behavior of $\tau-\mathrm{MnAl}$ are magnetized along the c-axis of the tetragonal structure. The much larger out-of-plane coercivity compared to the in-plane one indicates that the majority of the moments are pointing out-of -plane, i.e., the direction of c-axis is perpendicular to the thin film surface. This conclusion could also be confirmed by the high resolution XRD scan with the Ge(220)x2 monochrometer filter. As shown in Figure 8.10, the fact that only $\mathrm{MnAl}(001)$ and $\mathrm{MnAl}(002)$ peaks could be observed suggested again that the majority of the crystal structures had the (001) orientation with the c-axis perpendicular to the film plane. There might be a small portion of the (100) orientation with easy axis in-plane, as indicated by the in-plane hysteresis. However, the fraction is too small for the high-resolution XRD scan to pick up the signals of the fundamental (200) peak and the superstructure (100) peak. 


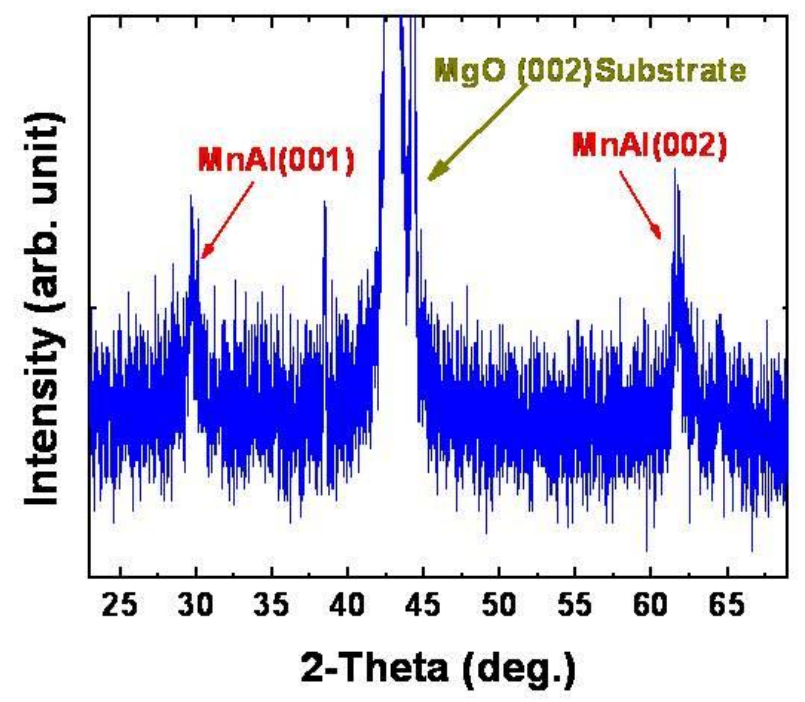

Figure 8.10 High resolution XRD scan of MnAl sample L092409

Another important parameter that we could obtain from the XRD scan is the chemical ordering parameter $\mathrm{S}$. Inside the tetragonal $\mathrm{L} 1_{0}$ phase, this parameter describes how well the alternating layered $\mathrm{Mn}$ and $\mathrm{Al}$ atoms are arranged within their own lattice planes. As I described in Figure 8.1 at the beginning of this chapter, if all the Mn atoms are sitting in their own position at the corners of the tetragonal structure and $\mathrm{Al}$ atoms in the body center $(1 / 2,1 / 2,1 / 2)$, this ideal configuration refers to the perfect chemical ordering state with $\mathrm{S}=1$. If the $\mathrm{Mn}$ and $\mathrm{Al}$ atoms change their positions and sit in each other's site, the chemical ordering will get reduced to some value smaller than 1, depending how disordered the $\mathrm{Mn}$ and $\mathrm{Al}$ atoms become. Basically, the $\mathrm{S}$ value is proportional to the intensity ratio of the superstructure (001) and fundamental (002) peaks. The detailed calculation method for the chemical ordering can be found in Ref. [89]. High quality $\tau$ - 
MnAl with good chemical ordering is essential for its technological applications, since the anisotropy and coercivity values are directly related to the chemical ordering state [90]. The other key parameter of $\tau-\mathrm{MnAl}$ thin films that is critical for its practical application is the saturation magnetization value $\mathrm{M}_{\mathrm{S}}$. As mentioned in the spin transfer torque background theory part, the $\mathrm{M}_{\mathrm{S}}$ value affects the switching current directly according to the equation 2.6. So here I would like to investigate the two key parameters ( $\mathrm{S}$ and $\mathrm{M}_{\mathrm{S}}$ ) by studying their film thickness dependence to further our understanding, and I believe this study will give us more insight about how to tailor the key properties of $\tau$ MnAl for its technological applications.

In this study, a set of three samples with different thickness were prepared by our RBTIBD system. The three films were deposited with same process conditions except the deposition time of 5, 10 and 20 min respectively, with the corresponding nominal thickness around 12, 22 and $40 \mathrm{~nm}$ by X-ray reflectivity measurement. Before any characterization, all three samples were annealed at our vacuum furnace at $400^{\circ} \mathrm{C}$ for 30 min without applying external field. The $\mathrm{M}_{\mathrm{S}}$ values were extracted from the VSM loop measurements, and the chemical ordering value $S$ values were calculated based on the intensity ratio of the $\mathrm{MnAl}(001)$ and (002) peaks from the XRD scans. The dependences on film thickness of the $S$ and $M_{S}$ values are plotted here in Figure 8.11. 


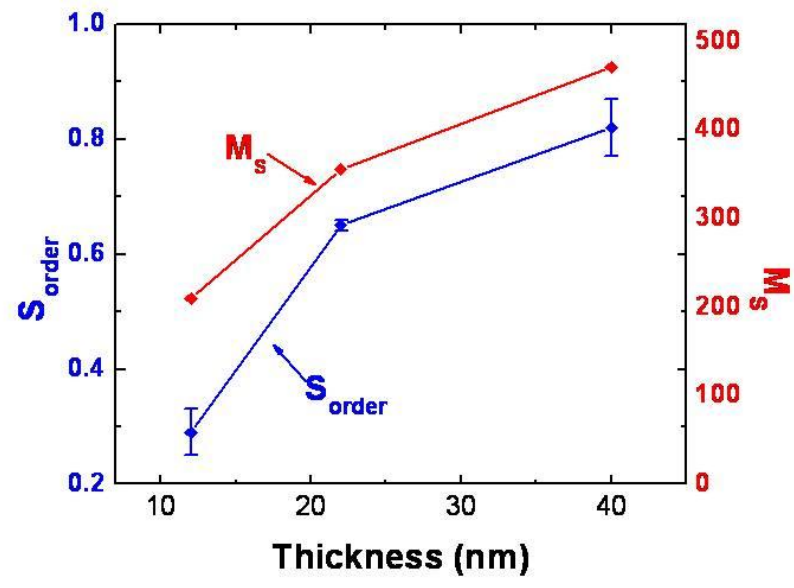

Figure 8.11 The chemical ordering $S$ and saturation magnetization $M_{S}$ dependence on the $\tau$-MnAl

\section{film thickness}

The blue curve in Figure 8.11 represents the chemical ordering $\mathrm{S}$ dependence on the film thickness. It clearly shows that when increasing the film thickness, the corresponding chemical ordering also gets increased. This is probably due to the strain relaxation of the films with larger thickness. Basically, the thinner the film is, the more significant the strain effect becomes. The larger strain effect will cause more intermixing of the $\mathrm{Mn}$ and $\mathrm{Al}$ atoms, which leads to the degradation of the chemical ordering. With larger thickness, the strain effect becomes less significant and more $\mathrm{Mn}$ and $\mathrm{Al}$ atoms will sit at their own sites. This makes more $\mathrm{Mn}$ and $\mathrm{Al}$ atoms to be confined in the layer by layer $\mathrm{L} 1_{0}$ superlattice structure with higher chemical ordering.

The red curve in Figure 8.11 represents the $\mathrm{M}_{\mathrm{S}}$ dependence on the film thickness. It shows a very similar trend as the chemical ordering dependence, with thicker film showing higher $\mathrm{M}_{\mathrm{S}}$ values. So a correlation between the chemical ordering and the saturation magnetization could be established here. To understand this correlation 
qualitatively, let us go back to the tetragonal crystals structure of $\tau$-MnAl, shown here again in Figure 8.12. As I mentioned before, the perfect chemical ordering refers to the state where $\mathrm{Mn}$ and $\mathrm{Al}$ atoms sit in their own position with a layer by layer structure. However, in reality, factors like the external strain or defects inside the crystal structure cause some random $\mathrm{Mn}$ and $\mathrm{Al}$ atoms to swap their positions. As illustrated in Figure 8.11, the $\mathrm{Mn}$ atoms sit on the positions originally occupied by $\mathrm{Al}$ and will be antiferromagnetically coupled with the rest of Mn atoms on their original positions. With such a configuration, the magnetization of $\tau$-MnAl not only loses the contribution from the Mn sitting at the corner, but also gets further reduced by the negative moment from the antiferromagnetically coupled $\mathrm{Mn}$ atom in the body center. This explains why the decrease of chemical ordering always comes together with the reduction of the saturation magnetization.
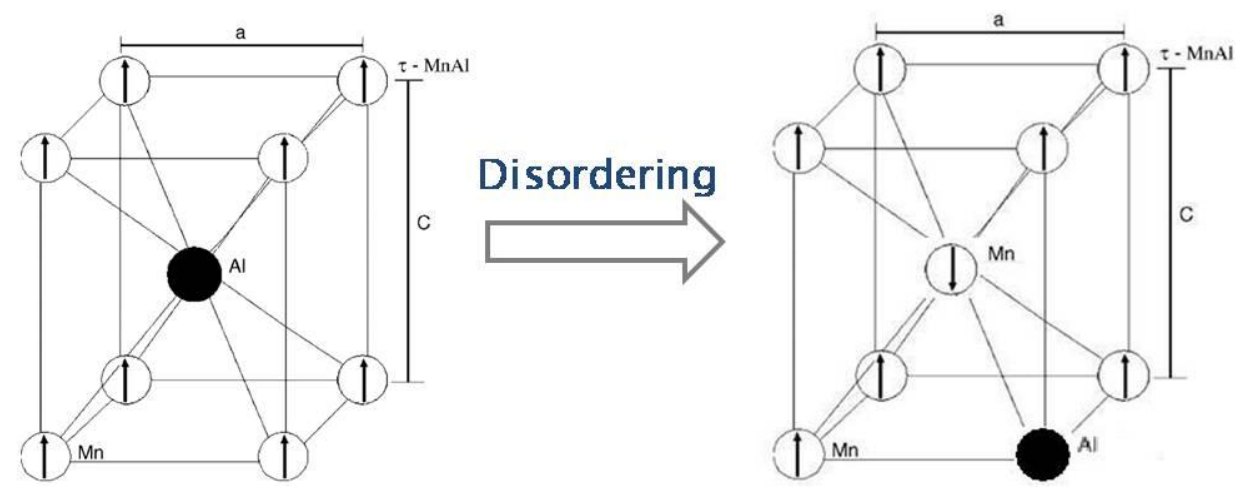

Figure 8.12 The illustration of the disordering process of $\tau-\mathrm{MnAl}$ by swap of the $\mathrm{Mn}$ and $\mathrm{Al}$ atom positions, with the wrong positioned Mn antiferromagnetically coupled with the rest Mn matrix. 


\subsubsection{Annealing effects on $\tau-\mathrm{MnAl}$ properties}

The previous study showed that for ambient temperature growth of thin film MnAl, the post-deposition heat treatment is essential to obtain the strong magnetic $\tau$ phase [84]. Our own experiments verified this conclusion by showing in Figure 8.12 a representative MnAl sample both in as-deposited state showing no magnetic signal at all and the strong magnetic $\tau$-MnAl state after vacuum annealing the same sample at $400^{\circ} \mathrm{C}$ for $30 \mathrm{~min}$.

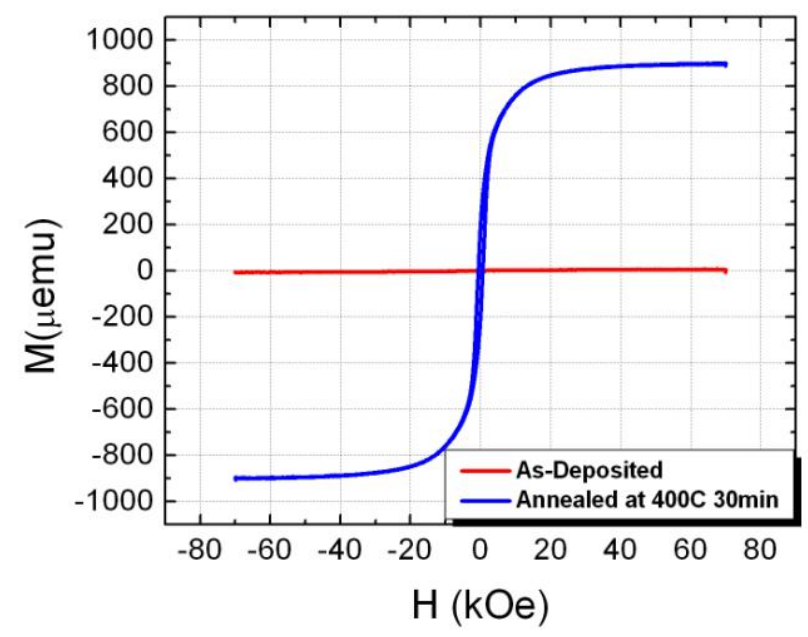

Figure 8.13 $\mathrm{M}(\mathrm{H})$ loops of a representative $\mathrm{MnAl}$ thin film sample both before and after $400^{\circ} \mathrm{C} 30$ min annealing treatment

At the initial stage of our study, all the post-deposition annealing was carried out in our home-built regular vacuum furnace. To further our study of the annealing effect, I also applied another annealing method called Rapid Thermal Annealing (RTA). Our regular annealing furnace has a maximum temperature ramping rate around $5^{\circ} \mathrm{C} / \mathrm{min}$, and the natural cooling down rate is even slower. Usually it takes more than 3 hours to 
cooling down the furnace from $400^{\circ} \mathrm{C}$ to RT. While in the other hand, the RTA furance features much higher heating and cooling rate, and the specific parameters of the two different furnaces are summarized in the following table.

Table 8-3 Annealing parameters of two vacuum furnace (A) regular (B) rapid thermal annealing

\begin{tabular}{|l|c|c|}
\hline Annealing Parameters & (A) Regular & (B) RTA \\
\hline Heating Rate & $5^{\circ} \mathrm{C} / \mathrm{min}$ & $2400^{\circ} \mathrm{C} / \mathrm{min}$ \\
\hline Cooling Rate & $2{ }^{\circ} \mathrm{C} / \mathrm{min}$ & $100{ }^{\circ} \mathrm{C} / \mathrm{min}$ \\
\hline Base Pressure (Torr) & $1 \times 10^{-5}$ & $8 \times 10^{-6}$ \\
\hline
\end{tabular}

To compare the annealing effect of the regular annealing and RTA, I selected two representative samples with different thickness and applied the same annealing conditions. The annealing conditions and the measured saturation magnetization value $\mathrm{M}_{\mathrm{S}}$ are summarized in the following table:

Table 8-4 Comparison of the two annealing methods (regular annealing and rapid thermal annealing) on two representative samples with different thickness

\begin{tabular}{|l|c|c|}
\hline Annealing Parameters & (A) Regular & (B) RTA \\
\hline Time (min) & 30 & 30 \\
\hline Temperature $\left({ }^{\circ} \mathrm{C}\right)$ & 400 & 400 \\
\hline $\mathrm{M}_{\mathrm{S}}(\mathrm{emu} / \mathrm{cc})(12 \mathrm{~nm}$ sample $)$ & $\mathbf{2 1 0}$ & $\mathbf{2 0 0}$ \\
\hline $\mathrm{M}_{\mathrm{S}}(\mathrm{emu} / \mathrm{cc})(120 \mathrm{~nm}$ sample $)$ & $\mathbf{3 0 0}$ & $\mathbf{5 6 3}$ \\
\hline
\end{tabular}


When keeping the same annealing time and temperature for the two different annealing methods, the major difference left is the different heating/cooling rate. The much slower heating and cooling rate means that the amount of total effective annealing energy absorbed by the samples under regular annealing would be much larger compared to the case of RTA. From the above Table $8-4$ we can see that for the thin sample ( $12 \mathrm{~nm})$, the regular annealing and RTA resulted in very close $\mathrm{M}_{\mathrm{S}}$ values, and both of them are much lower than the that of the thicker samples $(\sim 120 \mathrm{~nm})$. This might indicate that for the thin film samples, $30 \mathrm{~min}$ at $400^{\circ} \mathrm{C}$ annealing might be more than enough to complete the phase transformation to the magnetic $\tau$ phase of MnAl. After reaching the optimized state in $\tau$ phase of MnAl, the continued annealing caused more inter-diffusion of $\mathrm{Mn}$ and $\mathrm{Al}$ atoms to each others' crystal planes within the tetragonal structure. This led to the degradation of the $\tau$-MnAl chemical ordering. As I pointed out in section 8.3.4, the chemical ordering is correlated with the $\mathrm{M}_{\mathrm{S}}$ value, so after such strong annealing, in both cases, the MnAl films reached a certain type of saturated state with low chemical ordering and small $\mathrm{M}_{\mathrm{S}}$ values, and such degraded states were not sensitive to the heat treatment any longer.

While for thicker MnAl samples ( $120 \mathrm{~nm}$ ), much higher $\mathrm{M}_{\mathrm{S}}$ values were obtained for the sample under RTA than regular annealing. The reduced $\mathrm{M}_{\mathrm{S}}$ with regular annealing, which essentially means larger amount of annealing energy, tells us that again that $30 \mathrm{~min}$ annealing at $400^{\circ} \mathrm{C}$ is more than enough for the phase transformation to the magnetic $\tau$ phase of $\mathrm{MnAl}$, and the excessive heating with regular annealing compared with RTA caused more chemical disordering in the thick MnAl sample as well. That is why RTA yielded high $\mathrm{M}_{\mathrm{S}}$ on the thick $\mathrm{MnAl}$ sample. This initial study also leads to our 
argument that the optimum annealing condition is very sensitive to the sample volume. So for sample with different thicknesses, different annealing conditions need to be applied to achieve the optimized $\mathrm{M}_{\mathrm{S}}$ value.

To study the annealing effect systematically, I prepared two sets of samples with two different thicknesses (12 $\mathrm{nm}$ and $120 \mathrm{~nm})$ and both the annealing temperature and time dependence were investigated. Since RTA has much quicker heating/cooling rate, it was chosen as the annealing method in this study so that I will have better control of the annealing time and do not have to worry about the inaccuracy due to excessive heating caused by the slow ramping rate.

First I studied the annealing temperature dependence of samples with two different thicknesses by fixing the RTA time at $1 \mathrm{~min}$. The measured $\mathrm{M}_{\mathrm{S}}$ dependence on the RTA temperature is summarized in the following Figure 8.14. Two totally opposite trends could be observed for the two sample sets with different thickness. For thin samples $\sim 12$ $\mathrm{nm}$, higher annealing temperature led to lower $\mathrm{M}_{\mathrm{S}}$ values. This is probably because for such thin films, even $1 \mathrm{~min}$ annealing at $300^{\circ} \mathrm{C}$ is sufficient to complete the $\mathrm{MnAl}$ transformation to its magnetic $\tau$ phase from other nonmagnetic phases. Higher annealing temperature will presumably cause more intermixing of $\mathrm{Mn}$ and $\mathrm{Al}$ atoms, thus resulting in lower chemical ordering as well as reduced $\mathrm{M}_{\mathrm{S}}$ values. While for the thicker films ( $120 \mathrm{~nm}$ ), the opposite may indicate that $1 \mathrm{~min}$ annealing even at $500^{\circ} \mathrm{C}$ is not enough for the full transformation to the magnetic $\tau$ phase across the whole film. With higher annealing temperature, more amount of $\tau$-MnAl gets formed showing larger magnetic signal. The continuing increase of $\mathrm{M}_{\mathrm{S}}$ even at $500^{\circ} \mathrm{C}$ indicates that through further tuning of the annealing temperature or time duration, it would reach an optimum point where the 
phase transition to $\tau$-MnAl for such thick film becomes fully completed and an even higher $\mathrm{M}_{\mathrm{S}}$ value would be expected.

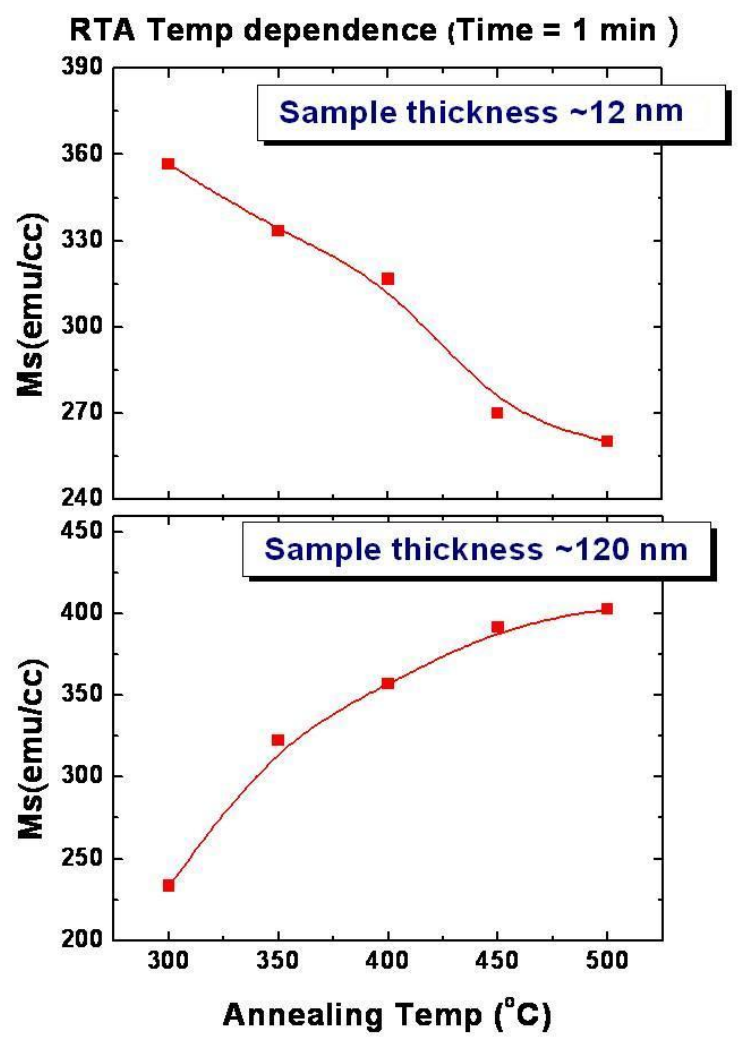

Figure 8.14 RTA temperature dependence of two sets of MnAl thin film samples with different thicknesses. The annealing time was kept at 1 min.

To further study the annealing effect and to confirm our argument that the optimized annealing condition is very sensitive to the sample thickness (i.e. the volume, when all the samples in our study were grown on $5 \times 5 \mathrm{~mm} \mathrm{MgO}(001)$ substrates), I also studied the annealing time dependence on the two sample sets with different thicknesses by fixing the RTA temperature at $400^{\circ} \mathrm{C}$. The measured $\mathrm{M}_{\mathrm{S}}$ dependence on the RTA time is summarized in the following Figure 8.15. 


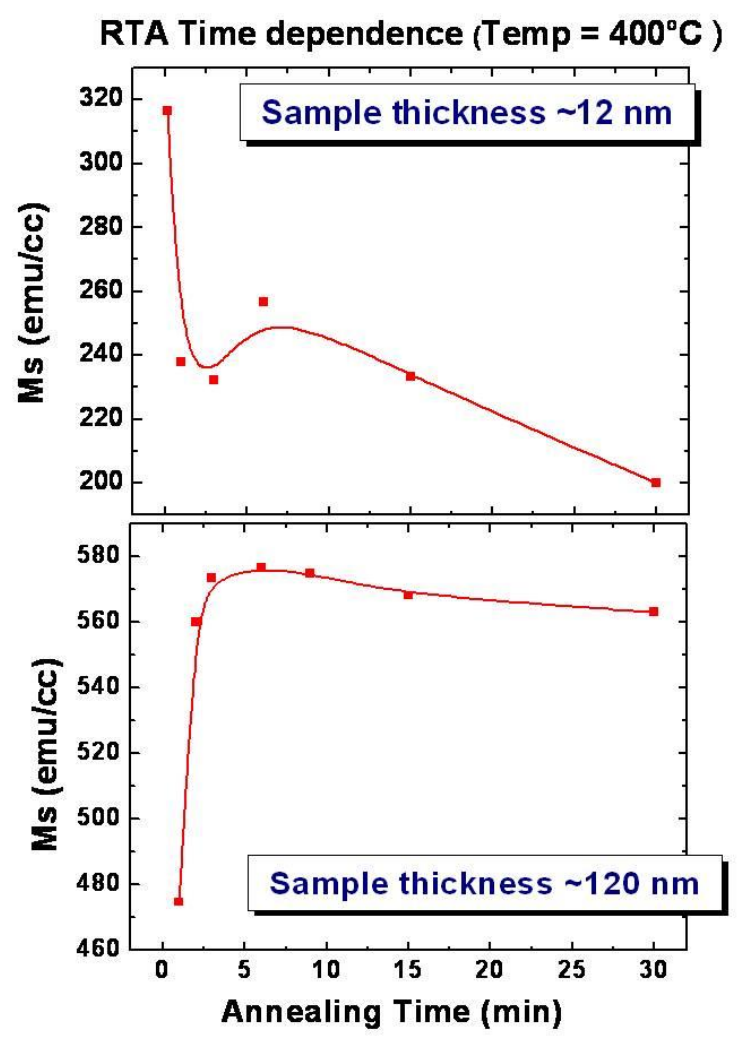

Figure 8.15 RTA time dependence of two sets of MnAl thin film samples with different thicknesses. The annealing temperature was kept at $400^{\circ} \mathrm{C}$.

Two different annealing time dependencies were observed in Figure 8.15 for samples with different thicknesses. First, for thin film $(\sim 12 \mathrm{~nm})$ samples, our analysis based on the annealing temperature dependence in Figure 8.14 stated that the annealing requirement for the small thickness (volume) was very low and even 1 min annealing at $300^{\circ} \mathrm{C}$ was more than enough for the full transformation to magnetic $\tau$ phase of MnAl. This argument is again confirmed by the top plot in Figure 8.15. It shows that for the thin samples with fixed annealing temperature, the highest $\mathrm{M}_{\mathrm{S}}$ was obtained with the shortest annealing time (10 seconds) and longer annealing time resulted in lower $\mathrm{M}_{\mathrm{S}}$, indicating 
that 10 seconds annealing at $400^{\circ} \mathrm{C}$ should be sufficient to complete the $\tau$ phase transition across the thin film and over annealing caused more intermixing of $\mathrm{Mn}$ and $\mathrm{Al}$ atoms, leading to the reduced chemical ordering as well as smaller $\mathrm{M}_{\mathrm{S}}$ values. However, the same 10 seconds annealing at $400^{\circ} \mathrm{C}$ didn't result in the highest $\mathrm{M}_{\mathrm{S}}$ for the thicker samples. This is probably due to its much larger volume and the transition to $\tau$-MnAl was only partially completed. The annealing temperature was optimized at around 6 min with the highest $\mathrm{M}_{\mathrm{S}} \sim 576 \mathrm{emu} / \mathrm{cc}$, much larger than the highest $\mathrm{M}_{\mathrm{S}}$ value ( 400 emu/cc) reported previously on $\tau-\mathrm{MnAl}$ thin films by sputtering [84]. The annealing time dependence study confirms again our argument that the optimum annealing condition is very sensitive to the sample volume, and different annealing conditions need to be applied to achieve the optimized $\mathrm{M}_{\mathrm{S}}$ value for samples with different thickness.

\subsection{Summary}

In this chapter, I started the experimental study of deposition MnAl thin films by exploring various growth templates. $\mathrm{MgO}(001)$ was identified as the best template with good lattice matching to support the growth of $\tau$-phase MnAl with perpendicular magnetic anisotropy (PMA). This discovery fits perfectly into our objective of seeking novel materials with PMA and other unique properties as the ferromagnetic (FM) electrode of MTJs with perpendicular magnetization, since $\mathrm{MgO}(001)$ is so far the best choice as the tunnel barrier material and high quality $\tau$-MnAl on $\mathrm{MgO}(001)$ with PMA was successfully grown by our RBTIBD system in this study. Extensive magnetic and structural characterization using VSM and XRD revealed the epitaxial growth 
relationship between the $\mathrm{MgO}(001)$ template and the $\tau-\mathrm{MnAl}(001)$ structure. Meanwhile, the correlation between the magnetic (saturation magnetization $\mathrm{M}_{\mathrm{S}}$ ) and the structural (chemical ordering) properties was established through the study of a series of samples with different thicknesses. The effect of heat treatment by regular annealing and RTA was studied, and RTA was proven to be the preferred method of choice with better annealing parameter control. Detailed RTA studies on two sets of samples with different thickness led to the conclusion that the optimum annealing condition is very sensitive to the sample volume, so different annealing conditions need to be applied to achieve the optimized $\mathrm{M}_{\mathrm{S}}$ value for samples with different thickness. This study allows us to tune the magnetic and the related structural properties of $\tau$-MnAl thin films, which is very important when incorporating the $\tau$-MnAl as the FM electrode into MTJs for Spin Torque Transfer MRAM applications. 


\section{Concluding remarks}

\subsection{Summary of scientific observations}

In summary, this dissertation explored the development of magnetic tunnel junctions (MTJs) from both structural perspective and material aspect, both with the purpose to improve the MTJ performance for applications in STT-RAM technologies.

The first major task of this dissertation was to establish the benchmark for MTJ deposition by our BTIBD system. Before the full stack deposition, exchange biased (EB) bilayers (FM/AFM) were first deposited, and it was found that the uncompensated spins are created favorably when an AFM is deposited on a magnetized FM layer. This finding is important for building full MTJ stacks and it led us to the creation of the FM/AFM/FM trilayer, in which the physics of the propagation of EB coupling was investigated.

Another critical step in building a full MTJ stack is the formation of ultrathin insulating barrier layer, and the barrier layer over-oxidation is a common problem to cause reduced TMR performance. If the barrier layer is over-oxidized, the surface of the underlying FM electrode (usually Co rich alloys) will be oxidized and antiferromagnetic CoO layer will be produced. Together with the FM electrode, the AFM/FM EB coupling system will be formed. Therefore, the EB coupling phenomenon could be used as a method to detect the over-oxidation of barrier layer, so that the precious control of the barrier layer oxidation could be achieved. The parameters associated with the EB coupling were also studied to further the understanding of electrode/barrier interface profile during the annealing process. 
Previous simulation work on the adatom energy impact showed that high adatom energy could produce flat thin film surface, but also cause strong multilayer mixing around the interface. By tuning the bias voltage which directly controls the adatom energy during the MTJ layer deposition, the impact of high adatom energy on thin film surface morphology and interface profile was confirmed experimentally. Combining both simulation and our experimental work, detailed strategies of optimizing the MTJ multilayer interface quality was proposed to enhance the MTJ performance for STTRAM applications.

After the above mentioned preparatory work, MTJs with AlOx and $\mathrm{MgO}$ barriers were deposited, with the barrier layer formed using multi-step natural oxidation method. High TMR around 70\% was achieved for MgO-MTJs after various optimizations including barrier and electrode layer thicknesses, annealing conditions, as well as the bias voltage control of adatom energies during the sputtering process. Also, some preliminary work on double barrier MTJs demonstrated the capability of making DBMTJs with either parallel or antiparallel pinned layers by the BTIBD system.

To improve the MTJ performance from the material perspective, $\tau$ phase MnAl thin film was synthesized and characterized comprehensively for its incorporation as the FM electrode with perpendicular magnetic anisotropy (PMA) into full MTJ stack with perpendicular magnetization. Initial deposition of MnAl onto various substrates revealed $\mathrm{MgO}(001)$ as the best growth template with good lattice matching, and high quality $\tau$ MnAl was grown on $\mathrm{MgO}(001)$ with proper annealing treatment. The thickness dependences of $\mathrm{MnAl}$ saturation magnetization $\left(\mathrm{M}_{\mathrm{S}}\right)$, magnetic anisotropy and structural chemical ordering were observed. And the correlation between $\mathrm{M}_{\mathrm{S}}$ and chemical ordering 
was established experimentally in conjunction with theoretical calculation from our collaborators. Rapid thermal annealing (RTA) was found to be a better heat treatment method compared to regular annealing with long ramping and cooling rate, and much higher $\mathrm{M}_{\mathrm{S}}$ than previously reported values was obtained from films made by our RBTIBD system with proper TRA treatment.

\subsection{Future research plans}

As to the future plan, new electrode materials with better spin transmission efficiency could easily be incorporated into the MTJ full stack based on the benchmark work of $\mathrm{MgO}-\mathrm{MTJ}$ structures. Electrodes like $\mathrm{CoFeB}$ doped with $\mathrm{Cr}$ which has the

potential to reduce the critical switching current density is currently being extensive studied in our group and ready to be incorporated into full MTJ stack simply by replacing the $\mathrm{CoFeB}$ layers in the original MTJ stacks developed in chapter 7. Other promising FM electrode materials with PMA that are currently being studied in the single film stage in our group include $\mathrm{GdCoFe}$ and $\mathrm{TbCoFe}$. Once the material characterization work is finished, they will be ready to incorporate into full MTJs using the BTIBD system.

$\tau$ phase MnAl as another very promising FM electrode with PMA and also good tunneling interface with $\mathrm{MgO}$ as predicted by theoretical calculation is being actively under investigation. I was able to make good quality $\mathrm{MnAl}$ thin film not only on $\mathrm{MgO}(001)$ substrates, but also on conducting metal seed layers like $\mathrm{Cr}$ with proper annealing treatment (results not shown in this dissertation). The successful growth of $\mathrm{MnAl}$ on metal seed layers is very important considering the task of building MTJ full 
stack with MnAl layers both above and below the $\mathrm{MgO}$ barrier layer. Future optimization of MnAl thin film includes trying alternative growth methods and addition of third element to better stabilize the MnAl metastable phase. For example, by depositing alternating layers of $\mathrm{Mn}$ and $\mathrm{Al}$, the preferred layer by layer $\mathrm{L} 1_{0}$ structure would be formed even before the annealing process, so better PMA would be expected with this technique [91]. Also, by slightly doping with carbon, MnAl-C films will be formed with better thermal stability [92]. Once the full optimization work for MnAl thin films is done, MnAl will be ready to incorporate into full MTJ stacks with perpendicular magnetization. 


\section{REFERENCE}

1. Miyazaki, T. and N. Tezuka, Giant magnetic tunneling effect in Fe/Al2O3/Fe junction. Journal of Magnetism and Magnetic Materials, 1995. 139(3): p. L231L234.

2. Moodera, J.S., et al., Large Magnetoresistance at Room Temperature in Ferromagnetic Thin Film Tunnel Junctions. Physical Review Letters, 1995. 74(16): p. 3273.

3. Engel, B.N., et al., A 4-Mb toggle MRAM based on a novel bit and switching method. Magnetics, IEEE Transactions on, 2005. 41(1): p. 132-136.

4. Berger, L., Emission of spin waves by a magnetic multilayer traversed by a current. Physical Review B, 1996. 54(13): p. 9353.

5. Slonczewski, J.C., Current-driven excitation of magnetic multilayers. Journal of Magnetism and Magnetic Materials, 1996. 159(1-2): p. L1-L7.

6. Stoner, E.C., Atomic moments in ferromagnetic metals and alloys with nonferromagnetic elements. Philosophical Magazine Series 7, 1933. 15(101): p. 1018 $-1034$.

7. $\quad$ Sciences, R.S.A.o. Scientific background on the Nobel Prize in Physics 2007:

"The Discovery of Giant Magnetoresistance". 2007 [cited; Available from: http://nobelprize.org/nobel_prizes/physics/laureates/2007/sci.html.

8. Mott, N.F., The Electrical Conductivity of Transistion Metals. Proc. Roy. Soc., 1936. A153: p. 699.

9. Zutic, I., J. Fabian, and S. Das Sarma, Spintronics: Fundamentals and applications. Reviews of Modern Physics, 2004. 76(2): p. 323.

10. Baibich, M.N., et al., Giant Magnetoresistance of (001)Fe/(001)Cr Magnetic Superlattices. Physical Review Letters, 1988. 61(21): p. 2472.

11. Binasch, G., et al., Enhanced magnetoresistance in layered magnetic structures with antiferromagnetic interlayer exchange. Physical Review B, 1989. 39(7): $\mathrm{p}$. 4828.

12. Myers, E.B., Dissertation: Manipulating nanomagnets with spin-polarized currents. 2002, Cornell University.

13. Tedrow, P.M. and R. Meservey, Spin Polarization of Electrons Tunneling from Films of Fe, Co, Ni, and Gd. Physical Review B, 1973. 7(1): p. 318.

14. Tedrow, P.M. and R. Meservey, Spin-Dependent Tunneling into Ferromagnetic Nickel. Physical Review Letters, 1971. 26(4): p. 192.

15. Meservey, R. and P.M. Tedrow, Spin-polarized electron tunneling. Physics Reports, 1994. 238(4): p. 173-243.

16. Maekawa, S. and U. Gafvert, Electron tunneling between ferromagnetic films. Magnetics, IEEE Transactions on, 1982. 18(2): p. 707-708.

17. Julliere, M., Tunneling between ferromagnetic films. Physics Letters A, 1975. 54(3): p. 225-226.

18. Wolf, S.A., et al., Spintronics: A Spin-Based Electronics Vision for the Future. Science, 2001. 294(5546): p. 1488-1495. 
19. Wang, D., et al., 70\% TMR at room temperature for SDT sandwich junctions with CoFeB as free and reference Layers. Magnetics, IEEE Transactions on, 2004. 40(4): p. 2269-2271.

20. Butler, W.H., et al., Spin-dependent tunneling conductance of $\mathrm{Fe}|\mathrm{MgO}| \mathrm{Fe}$ sandwiches. Physical Review B, 2001. 63(5): p. 054416.

21. Parkin, S.S.P., et al., Giant tunnelling magnetoresistance at room temperature with $\mathrm{MgO}$ (100) tunnel barriers. Nat Mater, 2004. 3(12): p. 862-867.

22. Yuasa, S., et al., Giant room-temperature magnetoresistance in single-crystal Fe/MgO/Fe magnetic tunnel junctions. Nat Mater, 2004. 3(12): p. 868-871.

23. Djayaprawira, D.D., et al., 230\% room-temperature magnetoresistance in $\mathrm{CoFeB} / \mathrm{MgO} / \mathrm{CoFeB}$ magnetic tunnel junctions. Applied Physics Letters, 2005. 86: p. 092502.

24. Lee, Y.M., et al., Effect of electrode composition on the tunnel magnetoresistance of pseudo-spin-valve magnetic tunnel junction with a $\mathrm{MgO}$ tunnel barrier. Applied Physics Letters, 2007. 90(21): p. 212507-3.

25. Wiese, N., PhD dissertation: Coupling phenomena and scalability of $\mathrm{CoFeB} / \mathrm{Ru} / \mathrm{CoFeB}$ sandwiches. 2006, Bielefeld University.

26. Nogues, J. and I.K. Schuller, Exchange bias. Journal of Magnetism and Magnetic Materials, 1999. 192(2): p. 203-232.

27. Yuasa, S., Giant Tunneling Magnetoresistance in MgO-Based Magnetic Tunnel Junctions. Journal of the Physical Society of Japan, 2008. 77(3): p. 031001.

28. Diao, Z., et al., Spin-transfer torque switching in magnetic tunnel junctions and spin-transfer torque random access memory. Journal of Physics: Condensed Matter, 2007(16): p. 165209.

29. Albert, F.J., et al., Spin-polarized current switching of a Co thin film nanomagnet. Applied Physics Letters, 2000. 77(23): p. 3809-3811.

30. Katine, J.A., et al., Current-Driven Magnetization Reversal and Spin-Wave Excitations in Co /Cu /Co Pillars. Physical Review Letters, 2000. 84(14): p. 3149.

31. Sun, J.Z., et al., Batch-fabricated spin-injection magnetic switches. Applied Physics Letters, 2002. 81(12): p. 2202-2204.

32. Huai, Y., et al., Observation of spin-transfer switching in deep submicron-sized and low-resistance magnetic tunnel junctions. Applied Physics Letters, 2004. 84(16): p. 3118-3120.

33. West, K.G., PhD dissertation: Exploration and Development of Novel Oxide Materials for Next Generation Spin Torque Transfer Heterostructures. 2009, University of Virginia.

34. Ohmori, H., T. Hatori, and S. Nakagawa, Perpendicular magnetic tunnel junction with tunneling magnetoresistance ratio of $64 \%$ using $\mathrm{MgO}$ (100) barrier layer prepared at room temperature. Journal of Applied Physics, 2008. 103(7): p. 3.

35. Meng, H. and J.-P. Wang, Spin transfer in nanomagnetic devices with perpendicular anisotropy. Applied Physics Letters, 2006. 88(17): p. 172506-3.

36. Shi, J., S. Tehrani, and M.R. Scheinfein, Geometry dependence of magnetization vortices in patterned submicron NiFe elements. Applied Physics Letters, 2000. 76(18): p. 2588-2590.

37. Mangin, S., et al., Current-induced magnetization reversal in nanopillars with perpendicular anisotropy. Nat Mater, 2006. 5(3): p. 210-215. 
38. Hylton, T.L., et al., Thin film processing by biased target ion beam deposition. Magnetics, IEEE Transactions on, 2000. 36(5): p. 2966-2971.

39. Quan, J., PhD Dissertation: Low Energy Ion Beam Assisted Growth of Metal Multilayers in Department of Material Science and Engineering. 2007, University of Virginia.

40. Quan, J.J., X.W. Zhou, and H.N.G. Wadley, Atomic assembly of metal surfaces and interfaces. Surface Science, 2006. 600(19): p. 4537-4547.

41. Zhou, X.W. and H.N.G. Wadley, Atomistic simulations of the vapor deposition of $\mathrm{Ni} / \mathrm{Cu} / \mathrm{Ni}$ multilayers: The effects of adatom incident energy. Journal of Applied Physics, 1998. 84(4): p. 2301-2315.

42. Zhou, X.W., et al., Atomic scale structure of sputtered metal multilayers. Acta Materialia, 2001. 49(19): p. 4005-4015.

43. Zhurin, V.V., et al., Biased target deposition. Journal of Vacuum Science \& Technology A: Vacuum, Surfaces, and Films, 2000. 18(1): p. 37-41.

44. Kaufman, H.R., R.S. Robinson, and R.I. Seddon, End-Hall ion source. Journal of Vacuum Science \& Technology A: Vacuum, Surfaces, and Films, 1987. 5(4): p. 2081-2084.

45. Kaufman, H.R. and L. Marton, Technology of Electron-Bombardment Ion Thrusters, in Advances in Electronics and Electron Physics. 1975, Academic Press. p. 265-373.

46. West, K., et al., Ferromagnetism in Rutile Structure Cr Doped VO2 Thin Films Prepared by Reactive-Bias Target Ion Beam Deposition. Journal of Superconductivity and Novel Magnetism, 2008. 21(2): p. 87-92.

47. West, K.G., et al., Growth and characterization of vanadium dioxide thin films prepared by reactive-biased target ion beam deposition. Journal of Vacuum Science \& Technology A: Vacuum, Surfaces, and Films, 2008. 26(1): p. 133-139.

48. Morisako, A., M. Matsumoto, and M. Naoe, Synthesis offerromagnetic tau phase of Mn-Al films by sputtering. Journal of Applied Physics, 1987. 61(8): p. 42814283.

49. Meiklejohn, W.H. and C.P. Bean, New Magnetic Anisotropy. Physical Review, 1956. 102(5): p. 1413.

50. Meiklejohn, W.H. and C.P. Bean, New Magnetic Anisotropy. Physical Review, 1957. 105(3): p. 904.

51. Yang, F.Y. and C.L. Chien, Spiraling Spin Structure in an Exchange-Coupled Antiferromagnetic Layer. Physical Review Letters, 2000. 85(12): p. 2597.

52. Zhou, S.M., K. Liu, and C.L. Chien, Exchange coupling and macroscopic domain structure in a wedged permalloy/FeMn bilayer. Physical Review B, 1998. 58(22): p. R14717.

53. Nikitenko, V.I., et al., Asymmetry in Elementary Events of Magnetization Reversal in a Ferromagnetic/Antiferromagnetic Bilayer. Physical Review Letters, 2000. 84(4): p. 765.

54. Ambrose, T. and C.L. Chien. Dependence of exchange coupling on antiferromagnetic layer thickness in NiFe/CoO bilayers. in The 7th joint MMMintermag conference on magnetism and magnetic materials. 1998. San Francisco, California (USA): AIP. 
55. Gokemeijer, N.J., T. Ambrose, and C.L. Chien, Long-Range Exchange Bias across a Spacer Layer. Physical Review Letters, 1997. 79(21): p. 4270.

56. Leung, C.W. and M.G. Blamire, Interaction between exchange-bias systems in $N i \_\{80\} F e_{-}\{20\} / F e_{-}\{50\} M n_{-}\{50\} / C o$ trilayers. Physical Review B, 2005. 72(5): p. 054429.

57. Leung, C.W. and M.G. Blamire, Interaction between ferromagnetic/antiferromagnetic systems across a common antiferromagnetic spacer. Journal of Applied Physics, 2003. 94(11): p. 7373-7375.

58. Ikeda, S., et al., Tunnel magnetoresistance of $604 \%$ at $300 \mathrm{~K}$ by suppression of Ta diffusion in $\mathrm{CoFeB} / \mathrm{MgO} / \mathrm{CoFeB}$ pseudo-spin-valves annealed at high temperature. Applied Physics Letters, 2008. 93(8): p. 3.

59. Jiang, L., et al., Large Tunnel Magnetoresistance of $1056 \%$ at Room Temperature in MgO Based Double Barrier Magnetic Tunnel Junction. Applied Physics Express, 2009. 2(8): p. 083002.

60. Read, J.C., P.G. Mather, and R.A. Buhrman, X-ray photoemission study of CoFeB/MgO thin film bilayers. Applied Physics Letters, 2007. 90(13): p. 1325033.

61. Velthuis, S.G.E.t., et al. Training effects and the microscopic magnetic structure of exchange biased Co/CoO bilayers. 2000: AIP.

62. Lu, Y., et al., A magnetometry study of Co oxidation in $\mathrm{Co} / \mathrm{MgO}$ bilayers grown by sputtering. Journal of Applied Physics, 2008. 104(7): p. 073907-5.

63. Hayakawa, J., et al., Dependence of Giant Tunnel Magnetoresistance of Sputtered $\mathrm{CoFeB/MgO/CoFeB} \mathrm{Magnetic} \mathrm{Tunnel} \mathrm{Junctions} \mathrm{on} \mathrm{MgO} \mathrm{Barrier} \mathrm{Thickness} \mathrm{and}$ Annealing Temperature. Japanese Journal of Applied Physics, 2005. 44(19): p. L587.

64. Vopsaroiu, M., et al., Growth rate effects in soft CoFe films. Magnetics, IEEE Transactions on, 2005. 41(10): p. 3253-3255.

65. Manzoor, S., et al. Grain-size effects in exchange-biased FeMn/NiFe bilayers. in 49th Annual Conference on Magnetism and Magnetic Materials. 2005. Jacksonville, Florida (USA): AIP.

66. Schrag, B.D., et al., Néel "orange-peel" coupling in magnetic tunneling junction devices. Applied Physics Letters, 2000. 77(15): p. 2373-2375.

67. Chen, E.Y., et al. Comparison of oxidation methods for magnetic tunnel junction material. 2000: AIP.

68. Worledge, D.C. and P.L. Trouilloud, Magnetoresistance measurement of unpatterned magnetic tunnel junction wafers by current-in-plane tunneling. Applied Physics Letters, 2003. 83(1): p. 84-86.

69. Yang, J.J., et al., Origin of the dependence of magnetoresistance on the composition of Co[sub 100 - x] Fe[sub x] electrodes in magnetic tunnel junctions. Journal of Applied Physics, 2008. 103(5): p. 056102-3.

70. Chen, W., et al., Effects of target bias voltage in magnetic tunnel junctions grown by ion beam deposition. Journal of Applied Physics, 2009. 106(1): p. 013905-4.

71. Pong, P.W.T., et al. Detection of pinholes in magnetic tunnel junctions by magnetic coupling. in Proceedings of the 52nd Annual Conference on Magnetism and Magnetic Materials. 2008. Tampa, Florida (USA): AIP. 
72. Ji Young, B., et al., Characteristics of the $A l\langle s u b>2</ s u b>O<s u b>3</ s u b\rangle$ barrier with CoFeB pinned Layer in magnetic tunnel junctions. Magnetics, IEEE Transactions on, 2005. 41(10): p. 2676-2678.

73. Moon, K.-S., Y. Chen, and Y. Huai. PtMn-based spin-dependent tunneling materials with thin alumina barrier fabricated by two-step natural oxidation. 2002: AIP.

74. Nagahama, T., et al., Spin-Dependent Tunneling in Magnetic Tunnel Junctions with a Layered Antiferromagnetic Cr(001) Spacer: Role of Band Structure and Interface Scattering. Physical Review Letters, 2005. 95(8): p. 086602.

75. Yuasa, S., T. Nagahama, and Y. Suzuki, Spin-Polarized Resonant Tunneling in Magnetic Tunnel Junctions. Science, 2002. 297(5579): p. 234-237.

76. Vadala, M., et al., Structural characterization and magnetic profile of annealed CoFeB/MgO multilayers. Journal of Applied Physics, 2009. 105(11): p. 113911-6.

77. Simmons, J.G., Generalized Formula for the Electric Tunnel Effect between Similar Electrodes Separated by a Thin Insulating Film. Journal of Applied Physics, 1963. 34(6): p. 1793-1803.

78. Diao, Z., et al., Spin transfer switching in dual MgO magnetic tunnel junctions. Applied Physics Letters, 2007. 90(13): p. 132508-3.

79. Huai, Y., et al., Spin transfer switching current reduction in magnetic tunnel junction based dual spin filter structures. Applied Physics Letters, 2005. 87(22): p. 222510-3.

80. Childress, J.R., et al., Fabrication and Recording Study of All-Metal Dual-SpinValve CPP Read Heads. Magnetics, IEEE Transactions on, 2006. 42(10): p. 24442446.

81. Kono, H., On the Ferromagnetic Phase in Manganese-Aluminum System. Journal of the Physical Society of Japan, 1958. 13(12): p. 1444-1451.

82. Zeng, Q., et al., Structural and magnetic properties of nanostructured Mn-Al-C magnetic materials. Journal of Magnetism and Magnetic Materials, 2007. 308(2): p. 214-226.

83. Koch, A.J.J., et al., New Material for Permanent Magnets on a Base of Mn and Al. Journal of Applied Physics, 1960. 31(5): p. S75-S77.

84. Huang, J.H., P.C. Kuo, and S.C. Shen, High coercivity and high saturation magnetization Mn-Al thin films. Magnetics, IEEE Transactions on, 1995. 31(5): p. 2494-2498.

85. Sands, T., et al., Epitaxial ferromagnetic tau-MnAl films on GaAs. Applied Physics Letters, 1990. 57(24): p. 2609-2611.

86. Rosier, T.M., Y.W. He, and N.A. El-Masry, Ferromagnetic [tau]-MnAl epitaxially grown on (100) GaAs substrates by pulsed laser deposition. Materials Letters, 1996. 26(4-5): p. 227-231.

87. Van Den Broek, J.J., et al., Phase transformations in pure and carbon-doped Al45Mn55 alloys. Acta Metallurgica, 1979. 27(9): p. 1497-1504.

88. Ding, Y.F., et al. Effect of lattice mismatch on chemical ordering of epitaxial L1[sub 0] FePt films. in 49th Annual Conference on Magnetism and Magnetic Materials. 2005. Jacksonville, Florida (USA): AIP.

89. Cebollada, A., R.F.C. Farrow, and M.F. Toney, Structure and Magnetic Properties of Chemically Ordered Magnetic Binary Alloys in Thin 
Film Form, in Magnetic Nanostructures H.S. Nalwa, Editor. 2002, American Scientific Publishers. p. 93-122.

90. Van Roy, W., et al., Degree of order and magnetic properties of [tau]-MnAl films. Journal of Magnetism and Magnetic Materials, 1995. 148(1-2): p. 97-98.

91. Duan, C.Y., et al., The structural and magnetic properties of [tau]-MnAl films prepared by Mn/Al multilayers deposition plus annealing. Materials Science and Engineering: B, 2009. 162(3): p. 185-188.

92. Bohlmann, M.A., J.C. Koo, and J.H. Wise, Mn-Al-C for permanent magnets (invited). Journal of Applied Physics, 1981. 52(3): p. 2542-2543. 